\title{
Ionization fraction and the enhanced sulfur chemistry in Barnard 1
}

\author{
A. Fuente ${ }^{1}$, J. Cernicharo ${ }^{2}$, E. Roueff ${ }^{3,4}$, M. Gerin ${ }^{3,4}$, J. Pety ${ }^{5}$, N. Marcelino ${ }^{6}$, R. Bachiller ${ }^{1}$, B. Lefloch ${ }^{7}$, \\ O. Roncero ${ }^{8}$, and A. Aguado 9 \\ 1 Observatorio Astronómico Nacional (OAN,IGN), Apdo 112, 28803 Alcalá de Henares, Spain \\ e-mail: a.fuente@oan.es \\ 2 Instituto de Ciencia de Materiales de Madrid, ICMM-CSIC, C/ Sor Juana Inés de la Cruz 3, 28049 Cantoblanco, Spain \\ ${ }^{3}$ CNRS UMR 8112, LERMA, Observatoire de Paris and École Normale Supérieure. 24 rue Lhomond, 75231 Paris Cedex 05, France \\ ${ }^{4}$ Sorbonne Universités, UPMC Univ. Paris 06, UMR8112, LERMA, 75005 Paris, France \\ 5 Institut de Radioastronomie Millimétrique, 300 rue de la Piscine, 38406 Saint Martin d'Hères, France \\ ${ }^{6}$ INAF, Osservatorio di Radioastronomia, via P. Gobetti 101, 40129 Bologna, Italy \\ 7 Institut de Planétologie et d'Astrophysique de Grenoble (IPAG) UMR 5274, Université UJF-Grenoble 1/CNRS-INSU, \\ 38041 Grenoble, France \\ 8 Instituto de Física Fundamental (IFF-CSIC), C.S.I.C., Serrano 123, 28006 Madrid, Spain \\ 9 Facultad de Ciencias, Unidad Asociada de Química-Física Aplicada CSIC-UAM, Universidad Autónoma de Madrid, \\ 28049 Madrid, Spain
}

Received 10 February 2016 / Accepted 7 May 2016

\begin{abstract}
Context. Barnard B1b has been revealed as one of the most interesting globules from the chemical and dynamical point of view. It presents a rich molecular chemistry characterized by large abundances of deuterated and complex molecules. Furthermore, this globule hosts an extremely young Class 0 object and one candidate for the first hydrostatic core (FHSC) proving the youth of this star-forming region.

Aims. Our aim is to determine the cosmic ray ionization rate, $\zeta_{\mathrm{H}_{2}}$, and the depletion factors in this extremely young star-forming region. These parameters determine the dynamical evolution of the core.

Methods. We carried out a spectral survey toward Barnard 1b as part of the IRAM large program "IRAM Chemical survey of sun-like star-forming regions" (ASAI) using the IRAM 30-m telescope at Pico Veleta (Spain). This provided a very complete inventory of neutral and ionic C-, N-, and S- bearing species with, from our knowledge, the first secure detections of the deuterated ions $\mathrm{DCS}^{+}$and $\mathrm{DOCO}^{+}$. We use a state-of-the-art pseudo-time-dependent gas-phase chemical model that includes the ortho and para forms of $\mathrm{H}_{2}$, $\mathrm{H}_{2}^{+}, \mathrm{D}_{2}^{+}, \mathrm{H}_{3}^{+}, \mathrm{H}_{2} \mathrm{D}^{+}, \mathrm{D}_{2} \mathrm{H}^{+}, \mathrm{D}_{2}$, and $\mathrm{D}_{3}^{+}$to determine the local value of the cosmic ray ionization rate and the depletion factors.

Results. Our model assumes $n\left(\mathrm{H}_{2}\right)=10^{5} \mathrm{~cm}^{-3}$ and $T_{k}=12 \mathrm{~K}$, as derived from our previous works. The observational data are well fitted with $\zeta_{\mathrm{H}_{2}}$ between $3 \times 10^{-17} \mathrm{~s}^{-1}$ and $10^{-16} \mathrm{~s}^{-1}$ and the elemental abundances $\mathrm{O} / \mathrm{H}=3 \times 10^{-5}, \mathrm{~N} / \mathrm{H}=6.4-8 \times 10^{-5}, \mathrm{C} / \mathrm{H}=$ $1.7 \times 10^{-5}$, and $\mathrm{S} / \mathrm{H}$ between $6.0 \times 10^{-7}$ and $1.0 \times 10^{-6}$. The large number of neutral/protonated species detected allows us to derive the elemental abundances and cosmic ray ionization rate simultaneously. Elemental depletions are estimated to be $\sim 10$ for $\mathrm{C}$ and $\mathrm{O}$, $\sim 1$ for $\mathrm{N}$, and $\sim 25$ for $\mathrm{S}$.

Conclusions. Barnard $\mathrm{B} 1 \mathrm{~b}$ presents similar depletions of $\mathrm{C}$ and $\mathrm{O}$ as those measured in prestellar cores. The depletion of sulfur is higher than that of $\mathrm{C}$ and $\mathrm{O}$, but not as extreme as in cold cores. In fact, it is similar to the values found in some bipolar outflows, hot cores, and photon-dominated regions. Several scenarios are discussed to account for these peculiar abundances. We propose that it is the consequence of the initial conditions (important outflows and enhanced UV fields in the surroundings) and a rapid collapse ( $\sim 0.1 \mathrm{Myr})$ that allows most S- and N-bearing species to remain in the gas phase to great optical depths. The interaction of the compact outflow associated with B1b-S with the surrounding material could enhance the abundances of S-bearing molecules, as well.
\end{abstract}

Key words. astrochemistry - stars: formation - ISM: molecules - ISM: individual objects: Barnard 1 - ISM: abundances

\section{Introduction}

Cosmic rays (hereafter CRs) play a leading role in the chemistry, heating, and dynamics of the interstellar medium (ISM). By ionizing their main component, molecular hydrogen, CRs interact with dense molecular clouds, and this process initiates a series of ion-molecule reactions that form compounds of increasing complexity to eventually build up the rich chemistry observed in dark clouds (see, e.g., Wakelam et al. 2010). Also, CRs represent an important heating source for molecular clouds. Collisions with interstellar molecules and atoms convert about half of the energy of primary and secondary electrons yielded by the ionization process into heat (Glassgold \& Langer 1973; Glassgold et al. 2012). Finally, the ionization fraction controls the coupling of magnetic fields with the gas, driving the dissipation of turbulence and angular momentum transfer, thus playing a crucial role in protostellar collapse and the dynamics of accretion disks (see Padovani et al. 2013, and references therein).

The cosmic ray ionization rate $\zeta_{\mathrm{H}_{2}}$ is the number of hydrogen molecule ionization per second produced by CRs. In absence of other ionization agents (X-rays, UV photons, J-type shocks), the ionization fraction is proportional to $\sqrt{\zeta_{\mathrm{H}_{2}}}$, which becomes the key parameter in the molecular cloud evolution (McKee 1989; Caselli et al. 2002). During the last 50 yr, values 


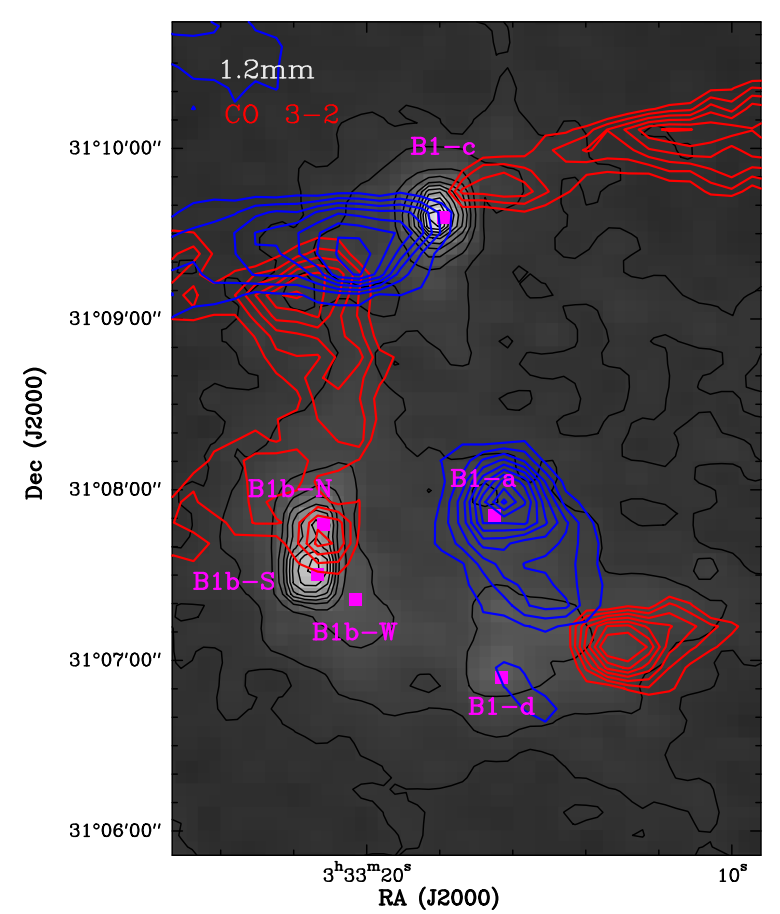

Fig. 1. Continuum map at $1.2 \mathrm{~mm}$ observed at the IRAM telescope with the MAMBO instrument (Daniel et al. 2013). The contours are $20 \mathrm{mJy} / \mathrm{beam}$ and $40 \mathrm{mJy} / \mathrm{beam}$ to $400 \mathrm{mJy} / \mathrm{beam}$ in steps of $40 \mathrm{mJy} /$ beam. Contours show the high velocity blue $\left(2.5\right.$ to $\left.4.5 \mathrm{~km} \mathrm{~s}^{-1}\right)$ and red ( 8.5 to $11.5 \mathrm{~km} \mathrm{~s}^{-1}$ ) emission of the $\mathrm{CO} 3 \rightarrow 2$ line as observed with the JCMT (project S12AC01).

of $\zeta_{\mathrm{H}_{2}}$ ranging from a few $10^{-18} \mathrm{~s}^{-1}$ to a few $10^{-16} \mathrm{~s}^{-1}$ have been observationally determined in diffuse and dense interstellar clouds (Galli \& Padovani 2015, and references therein). The cosmic ray ionization rate has usually been estimated from the $\left[\mathrm{HCO}^{+}\right] /[\mathrm{CO}]$ and/or $\left[\mathrm{DCO}^{+}\right] /\left[\mathrm{HCO}^{+}\right]$abundance ratios using simple analytical expressions (e.g., Guelin et al. 1977; Wootten et al. 1979; Dalgarno \& Lepp 1984). In recent studies, these estimates have been improved using comprehensive chemistry models (Caselli et al. 1999; Bergin et al. 1999). These models show that the gas ionization fraction is also dependent on the depletion factors that should be known to accurately estimate $\zeta_{\mathrm{H}_{2}}$ (Caselli et al. 1999).

Chemical modeling is required to constrain the elemental abundances and the cosmic ray ionization rate. We carried out a spectral survey toward Barnard $1 \mathrm{~b}$ as part of the IRAM large program "IRAM Chemical survey of sun-like star-forming regions" (ASAI; PIs: Rafael Bachiller, Bertrand Lefloch) using the IRAM 30-m telescope at Pico Veleta (Spain), which provided a very complete inventory of ionic species including $\mathrm{C}$-, $\mathrm{N}$-, and S-bearing compounds. The large number of neutral/protonated species allows us to derive the elemental abundances in the gas phase and cosmic ray ionization rate simultaneously, avoiding the uncertainty due to unknown depletion factors in the latter.

Barnard 1 (B1) belongs to the Perseus molecular cloud complex $(D=235 \mathrm{pc})$. The Barnard 1 dark cloud itself is subdivided into several dense cores at different evolutionary stages of star formation. While B1-a and B1-c are known to host class 0 sources associated with high velocity outflows (Hatchell et al. 2005, 2007), the B1-b core appears to be more quiet, although its western edge is interacting with an outflow that is possibly arising from sources B1-a or B1-d, which are both located 1' SW of B1-b (see a sketch in Fig. 1).
The $\mathrm{B} 1 \mathrm{~b}$ region was mapped in many molecular tracers, for example, $\mathrm{HCO}^{+}, \mathrm{CS}, \mathrm{NH}_{3},{ }^{13} \mathrm{CO}$ (Bachiller \& Cernicharo 1984; Bachiller et al. 1990), $\mathrm{N}_{2} \mathrm{H}^{+}$(Huang \& Hirano 2013), $\mathrm{H}^{13} \mathrm{CO}^{+}$(Hirano et al. 1999), or $\mathrm{CH}_{3} \mathrm{OH}$ (Hiramatsu et al. 2010; Öberg et al. 2010). Interferometric observations of Barnard 1b revealed that this compact core hosts two young stellar objects (YSOs), B1b-N and B1b-S (Huang \& Hirano 2013), and a third more evolved source, hereafter B1b-W, with deep absorption from ices (Jørgensen et al. 2006). The two first sources are deeply embedded in the surrounding protostellar envelope, which seems essentially unaffected by the embedded sources as shown by the large column density, $N\left(\mathrm{H}_{2}\right) \sim 7.6 \times 10^{22} \mathrm{~cm}^{-2}$ (Daniel et al. 2013), and cold kinetic temperature, $T_{K}=12 \mathrm{~K}$ (Lis et al. 2010). Based on Herschel fluxes and subsequent SED modeling, Pezzuto et al. (2012) concluded that B1b-N and $\mathrm{B} 1 \mathrm{~b}-\mathrm{S}$ were younger than Class 0 sources and proposed these as first hydrostatic core (FHSC) candidates. Using NOEMA, Gerin et al. (2015) obtained high resolution maps of the protostellar ouflows and their envelope, confirming the young ages of B1b-N and B1b-S ( 1000 yr and $~ 3000$ yr) and the high density of the surrounding core $\left(\sim\right.$ a few $10^{5} \mathrm{~cm}^{-3}$ for the outflowing gas).

From the chemical point of view, B $1 \mathrm{~b}$ is characterized by a rich chemistry. Indeed, many molecules were observed for the first time in this object, like HCNO (Marcelino et al. 2009) or $\mathrm{CH}_{3} \mathrm{O}$ (Cernicharo et al. 2012). Additionally, B1b shows a high degree of deuterium fractionation and has been associated with first detections of multiply deuterated molecules, such as $\mathrm{ND}_{3}$ (Lis et al. 2002) or $\mathrm{D}_{2} \mathrm{CS}$ (Marcelino et al. 2005), which is consistent with the expected chemistry in a dense and cold core.

\section{Observations}

We carried out a spectral survey toward B1b $\left(\alpha_{\mathrm{J} 2000}=\right.$ $03 \mathrm{~h} 33 \mathrm{~m} 20.8 \mathrm{~s}, \delta_{\mathrm{J} 2000}=31^{\circ} 07^{\prime} 34^{\prime \prime}$.0) using the IRAM $30-\mathrm{m}$ telescope at Pico Veleta (Spain). This position is in between the two FHSC candidates B1b-N and B1b-S. The two protostellar cores lie inside the HPBW of the $30-\mathrm{m}$ telescope at $3 \mathrm{~mm}$, but they are missed at $1 \mathrm{~mm}$ wavelengths (see Fig. 2).

The $3 \mathrm{~mm}$ observations were performed between January and May 2012 (see Cernicharo et al. 2012), which used the narrow mode of the FTS allowing a higher spectral resolution of $50 \mathrm{kHz}$. The observing mode in this case was frequency switching with a frequency throw of $7.14 \mathrm{MHz}$, which removes standing waves between the secondary and the receivers. Several observing periods were scheduled on July and February 2013 and January and March 2014 to cover the 1 and $2 \mathrm{~mm}$ bands shown in Table 1. The Eight MIxer Receivers (EMIR) and the Fast Fourier Transform Spectrometers (FTS) with a spectral resolution of $200 \mathrm{kHz}$ were used for these observations. The observing procedure was wobbler switching with a throw of $200^{\prime \prime}$ to ensure flat baselines. In Table 1 we show the half power beam width (HPBW), spectral resolution, and sensitivity achieved in each observed frequency band. The whole dataset will be published elsewhere but this paper is centered on the ionic species and sulfur compounds.

We complemented the unbiased spectral survey with $175^{\prime \prime} \times$ $175^{\prime \prime}$ maps of the SO $2_{2} \rightarrow 1_{1}, \mathrm{C}^{34} \mathrm{~S} 3 \rightarrow 2, \mathrm{HCS}^{+} 2 \rightarrow 1, \mathrm{HC}^{18} \mathrm{O}^{+}$ $1 \rightarrow 0, \mathrm{HOCO}^{+} 4_{04} \rightarrow 3_{03}, \mathrm{DCO}^{+} 2 \rightarrow 1, \mathrm{D}^{13} \mathrm{CO}^{+} 2 \rightarrow 1, \mathrm{p}-\mathrm{H}_{2} \mathrm{CO}$ $2_{02} \rightarrow 2_{01}, \mathrm{p}-\mathrm{H}_{2}{ }^{13} \mathrm{CO} 2_{02} \rightarrow 2_{01}$ lines (see Fig. 2). These maps were simultaneously observed at 3 and $2 \mathrm{~mm}$ with a combination of the EMIR receivers and the Fourier transform spectrometers, which yields a bandwidth of $1.8 \mathrm{GHz}$ per sideband per polarization at a channel spacing of $49 \mathrm{kHz}$. The tuned frequencies were 

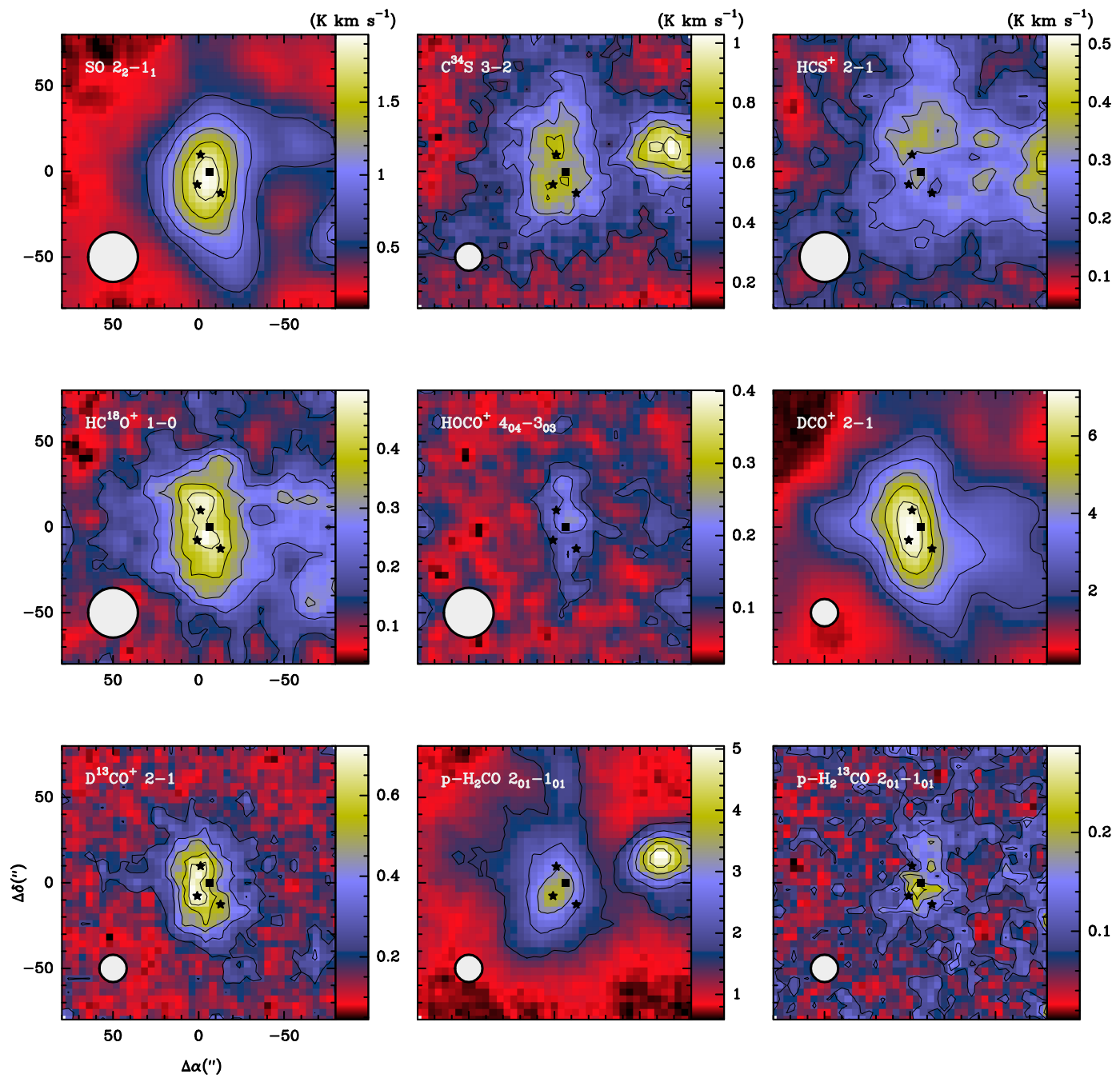

Fig. 2. Integrated intensity maps of the SO $2_{2} \rightarrow 1_{1}, \mathrm{C}^{34} \mathrm{~S} 3 \rightarrow 2, \mathrm{HCS}^{+} 2 \rightarrow 1, \mathrm{HC}^{18} \mathrm{O}^{+} 1 \rightarrow 0, \mathrm{HOCO}^{+} 4_{04} \rightarrow 33_{03}, \mathrm{DCO}^{+} 2 \rightarrow 1, \mathrm{D}^{13} \mathrm{CO}^{+} 2 \rightarrow 1$, $\mathrm{p}-\mathrm{H}_{2} \mathrm{CO} 2_{02} \rightarrow 2_{01}$, and $\mathrm{p}-\mathrm{H}_{2}^{13} \mathrm{CO} 2_{02} \rightarrow 2_{01}$ lines. The HPBW of the 30-m telescope at each line frequency is drawn in the bottom-left corner of the panel. The stars indicate the positions of B1b-N, B1b-S and B1b-W. The black square shows the position targeted in the unbiased spectral survey.

Table 1. Observing and telescope parameters.

\begin{tabular}{ccccc}
\hline \hline Freq $(\mathrm{GHz})$ & $\Delta v\left(\mathrm{~km} \mathrm{~s}^{-1}\right)$ & $H P B W^{a}$ & $B_{\mathrm{eff}}$ & $\operatorname{rms}\left(T_{a}\right)(\mathrm{mK})$ \\
\hline $82.5-117.6$ & 0.1255 & $29^{\prime \prime}-21^{\prime \prime}$ & $0.81-0.78$ & $3-5^{b}$ \\
$130.0-172.7$ & 0.3424 & $19^{\prime \prime}-14^{\prime \prime}$ & 0.74 & $6-10^{c}$ \\
$200.6-275.9$ & 0.2839 & $12^{\prime \prime}-9^{\prime \prime}$ & $0.63-0.49$ & $5-10$ \\
\hline
\end{tabular}

Notes. ${ }^{(a)} H P B W(\operatorname{arcsec}) \approx 2460 / F r e q(G H z) ;{ }^{(b)} \mathrm{rms} \sim 240 \mathrm{mK}$ for $v>$ $114 \mathrm{GHz} ;{ }^{(c)} \mathrm{rms} \sim 500 \mathrm{mK}$ for $v>169 \mathrm{GHz}$.

85.55 and $144.90 \mathrm{GHz}$. We used the on-the-fly scanning strategy with a dump time of $0.5 \mathrm{~s}$ and a scanning speed of $10.9^{\prime \prime} / \mathrm{s}$ to ensure a sampling of at least three dumps per beam at the $17.8^{\prime \prime}$ resolution of the $2 \mathrm{~mm}$ lines. The $175^{\prime \prime} \times 175^{\prime \prime}$ region was covered using successive orthogonal scans along the RA and Dec axes. The separation between two successive rasters was $6.5^{\prime \prime}$ $(\sim \lambda / 2 \mathrm{D})$ to ensure Nyquist sampling at the highest observed frequency, i.e., $145 \mathrm{GHz}$. A common reference position located at offsets $\left(200^{\prime \prime}, 150^{\prime \prime}\right)$ was observed for 10 seconds every $77.5 \mathrm{~s}$. The typical IRAM $30 \mathrm{~m}$ position accuracy is $3^{\prime \prime}$.
The data reduction and line identification were carried out with the package GILDAS ${ }^{1}$ /CLASS software.

\section{Methodology}

Our goal is to use the database provided by the IRAM large program ASAI together with previous millimeter data from our group (Marcelino et al. 2005, 2009, 2010; Cernicharo et al. 2012, 2013; Daniel et al. 2013; Cernicharo et al. 2014; Gerin et al. 2015) and complementary mapping to accurately determine the fundamental chemical parameters.

Within our spectral survey we detected the following ions: $\mathrm{HCO}^{+}, \mathrm{H}^{13} \mathrm{CO}^{+}, \mathrm{HC}^{18} \mathrm{O}^{+}, \mathrm{HC}^{17} \mathrm{O}^{+}, \mathrm{DCO}^{+}, \mathrm{D}^{13} \mathrm{CO}^{+}, \mathrm{HOCO}^{+}$, $\mathrm{DOCO}^{+}, \mathrm{HCS}^{+}, \mathrm{SO}^{+}, \mathrm{HC}^{34} \mathrm{~S}^{+}, \mathrm{DCS}^{+}, \mathrm{HCNH}^{+}, \mathrm{H}_{2} \mathrm{COH}^{+}$, $\mathrm{HC}_{3} \mathrm{NH}^{+}, \mathrm{NNH}^{+}, \mathrm{NND}^{+}, \mathrm{N}^{15} \mathrm{NH}^{+}$, and ${ }^{15} \mathrm{NNH}^{+}$. This is probably the first secure detection of the deuterated ions $\mathrm{DCS}^{+}$ and $\mathrm{DOCO}^{+}$. A tentative detection of $\mathrm{DOCO}^{+}$was reported by Fechtenbaum \& Bontemps (2013) toward the massive protostar CIGX-N63. Daniel et al. (2013) modeled the emission of $\mathrm{NNH}^{+}$, $\mathrm{NND}^{+}, \mathrm{N}^{15} \mathrm{NH}^{+}$, and ${ }^{15} \mathrm{NNH}^{+}$ions in this core. We use their

1 See http://www.iram.fr/IRAMFR/GILDAS for more information about the GILDAS softwares (Pety 2005). 
results to compare with our chemical models without repeating the analysis. Our final list of detections is the most complete inventory of molecular ions observed in this source and, therefore, an unprecedented opportunity to improve our knowledge of the ionization fraction. The main destruction mechanism of the observed protonated ions is the rapid recombination with $e^{-}$to give back the neutral parent species. For this reason, the $\left[\mathrm{XH}^{+}\right] /[\mathrm{X}]$ ratios are very sensitive to the gas ionization fraction and are commonly used to estimate it. We also include the lines of the parent molecules in our analysis to compare with chemical models (see Table A.1). It is interesting to note that we have not detected $\mathrm{CO}^{+}$and $\mathrm{HOC}^{+}$. These ions react with molecular hydrogen and only reach significant abundances in the external layers of photon-dominated regions that are not fully molecular (see, e.g., Fuente et al. 2003; Rizzo et al. 2003). The non-detection of these ions mainly supports the interpretation that we are probing a dense molecular core in which the ionization is dominated by cosmic rays.

The second key ingredient in gas-phase chemical models is the depletion factors. The sensitivity of the chemistry to the elemental abundances in the gas phase was discussed by Graedel et al. (1982), who introduced the concept of "lowmetal" elemental abundances, in which the abundances of metals, silicon, and sulfur are depleted by a factor of 100 with respect to the values typically used until then (those observed in the diffuse cloud $\zeta$ Oph). This choice of elemental abundances provides a reasonable agreement with the observed molecular abundances in dark clouds (see review by Agúndez \& Wakelam 2013). In recent decades, there has been increasing evidence that these abundances are decreasing with density because molecules are frozen onto the grain surfaces and reach the lowest values in dense prestellar cores. This phenomenon is quantified by the socalled depletion factor, that is, the ratio between the total (ice plus gas) abundance and that observed in the gas phase. The depletion is defined for a specific molecule or for the total amount of atoms of a given element. The depletion factor of each species depends on the local physical conditions, time evolution, and binding energy. For a given molecule, the depletion factor is evaluated by measuring the gas-phase abundance as a function of the gas density within the core. Tafalla et al. (2006) have shown that $\mathrm{CO}$ presents a strong variation while the effect is moderate for $\mathrm{HCN}$ and almost null for $\mathrm{N}_{2} \mathrm{H}^{+}$.

Determining the depletion factor of a given element is more complex, since it depends on the gas chemical composition and, eventually, on the binding energy of the compound(s) that are the most abundant. To derive the elemental gas abundances of $\mathrm{C}, \mathrm{O}, \mathrm{N}$, and $\mathrm{S}$, we need to include the main reservoirs of these elements in our analysis. The $\mathrm{CO}$ abundance is four orders of magnitude larger than that of any other molecule except $\mathrm{H}_{2}$. Therefore, we can assume that in a dense core, such as B1b, all the carbon is locked in $\mathrm{CO}$. The main reservoirs of nitrogen are atomic nitrogen $(\mathrm{N})$ and molecular nitrogen $\left(\mathrm{N}_{2}\right)$, which are not observable. The nitrogen abundance can be derived by applying a chemical model and fitting the observed abundances of nitriles $\left(\mathrm{HCN}, \mathrm{HNC}\right.$, and $\mathrm{CN}$ ) and $\mathrm{N}_{2} \mathrm{H}^{+}$. The most abundant oxygenated molecules, $\mathrm{O}_{2}, \mathrm{H}_{2} \mathrm{O}$, and $\mathrm{OH}$, cannot be observed in the millimeter domain and the oxygen depletion factor has to be derived indirectly. In the case of sulfur, the abundances of S-bearing species are very sensitive to the $\mathrm{C} / \mathrm{O}$ gas-phase ratio, and hence the oxygen depletion factor. A complete chemical modeling is needed to have a reasonable estimate of the $\mathrm{O}$ and $\mathrm{S}$ depletion factors.

\section{Results}

Figure B.1 shows the spectra of the neutral and protonated species considered in this work. In all cases, the protonated and neutral species present similar line profiles suggesting that both come from the same region. All the lines show two narrow velocity components, at $\sim 6.5 \mathrm{~km} \mathrm{~s}^{-1}$ and $\sim 7.0 \mathrm{~km} \mathrm{~s}^{-1}$, respectively. We use the $30 \mathrm{~m}$ maps to explore the origin of the different species and the two velocity components. The integrated intensity maps of the SO $2_{2} \rightarrow 1_{1}, \mathrm{C}^{34} \mathrm{~S} 3 \rightarrow 2, \mathrm{HCS}^{+} 2 \rightarrow 1, \mathrm{HC}^{18} \mathrm{O}^{+}$ $1 \rightarrow 0, \mathrm{HOCO}^{+} 4_{04} \rightarrow 3_{03}, \mathrm{DCO}^{+} 2 \rightarrow 1, \mathrm{D}^{13} \mathrm{CO}^{+} 2 \rightarrow 1, \mathrm{p}-\mathrm{H}_{2} \mathrm{CO}$ $2_{02} \rightarrow 2_{01}$, and $\mathrm{p}-\mathrm{H}_{2}{ }^{13} \mathrm{CO} 2_{02} \rightarrow 2_{01}$ lines are shown in Fig. 2. The emission of the $\mathrm{HC}^{18} \mathrm{O}^{+}, \mathrm{HOCO}^{+}, \mathrm{C}^{34} \mathrm{~S}, \mathrm{DCO}^{+}, \mathrm{D}^{13} \mathrm{CO}^{+}$, and SO lines is concentrated in the elongated filament that contains B1b-N and B1b-S (see Fig. 2). The emission of the $\mathrm{N}_{2} \mathrm{H}^{+} 1 \rightarrow 0$ line, as reported by Daniel et al. (2013), is also concentrated in this dense filament. In the case of $\mathrm{HOCO}^{+}$, the elongated emission seems to be slightly shifted to the west although the map is too noisy to conclude. In all these cases, the line intensities are similar toward the $\mathrm{N}$ and $\mathrm{S}$ protostars. On the contrary, the emission of the o- $\mathrm{NH}_{2} \mathrm{D} 1_{11} \mathrm{~s} \rightarrow 0_{01}$ a line peaks toward $\mathrm{B} 1 \mathrm{~b}-\mathrm{N}$ (Daniel et al. 2013). Another exception is $\mathrm{H}_{2} \mathrm{CO}$ whose emission presents an intense peak toward B1b-S. The emission of the $\mathrm{HCS}^{+} 2 \rightarrow 1$ line presents a flat spatial distribution suggesting that this emission is coming from the cloud envelope.

Linewidths can also provide information about the origin of the molecular emission. On basis of low angular resolution observations, Bachiller et al. (1990) proposed a correlation between the linewidths and the depth into the B1 cloud with the narrowest lines $\left(\sim 1 \mathrm{~km} \mathrm{~s}^{-1}\right)$ coming from the highest extinction regions. Similar results were obtained by Daniel et al. (2013) on basis of the observation of $\mathrm{HCN}, \mathrm{HNC}, \mathrm{N}_{2} \mathrm{H}^{+}, \mathrm{NH}_{3}$, and their isotopologues. The line profiles were well reproduced by a collapsing dense core (infall velocity $=0.1 \mathrm{~km} \mathrm{~s}^{-1}$ ) with a turbulent velocity of $0.4 \mathrm{~km} \mathrm{~s}^{-1}$ surrounded by a static lower density envelope with a turbulent velocity of $1.5 \mathrm{~km} \mathrm{~s}^{-1}$. In Fig. 3, we show the spatial distribution of the linewidths for the set of maps shown in Fig. 2. In agreement with previous works, we find a clear decrease of the linewidth with the distance from the core center in the SO $2_{2} \rightarrow 1_{1}$ line. Linewidths are $\sim 2 \mathrm{~km} \mathrm{~s}^{-1}$ in the most external layer and decrease to $\sim 0.7 \mathrm{~km} \mathrm{~s}^{-1}$ toward the core center. However, the linewidths of the $\mathrm{HCS}^{+} 2 \rightarrow 1$ lines are $\sim 2 \mathrm{~km} \mathrm{~s}^{-1}$ all over the region without a clear pattern. This supports the interpretation of the $\mathrm{HCS}^{+}$emission coming from an external layer of the cloud. The only position where the linewidths of the $\mathrm{HCS}^{+}$line becomes $<1 \mathrm{~km} \mathrm{~s}^{-1}$ is toward the IR star $\mathrm{B} 1 \mathrm{~b}-\mathrm{W}$. For $\mathrm{HC}^{18} \mathrm{O}^{+}, \mathrm{DCO}^{+}$, and $\mathrm{D}^{13} \mathrm{CO}^{+}$, we do see a trend, with the linewidths decreasing with the decreasing radius from the core center. However, their linewidths are always $>1 \mathrm{~km} \mathrm{~s}^{-1}$ suggesting that the emission is not dominated by the innermost layers of the envelope. The linewidth of the $\mathrm{p}-\mathrm{H}_{2} \mathrm{CO}$ line is very constant over the whole core. This line is more likely to be optically thick and its emission is only tracing the outer layers of the core.

Figures 4 and 5 show the spectral maps of the $\mathrm{DCO}^{+} 2 \rightarrow 1$ and SO $2_{2} \rightarrow 1_{1}$ lines, respectively. The emission of $\mathrm{DCO}^{+}$extends from $\sim 5.5 \mathrm{~km} \mathrm{~s}^{-1}$ to $\sim 8.0 \mathrm{~km} \mathrm{~s}^{-1}$, while the SO emission is detected in a narrower velocity range between $\sim 5.9 \mathrm{~km} \mathrm{~s}^{-1}$ to $\sim 7.5 \mathrm{~km} \mathrm{~s}^{-1}$, i.e., SO is mainly coming from the $6.5 \mathrm{~km} \mathrm{~s}^{-1}$ component. The intensity peak at $6.5 \mathrm{~km} \mathrm{~s}^{-1}$ is shifted $\sim 5^{\prime \prime}$ west from the peak at $7.0 \mathrm{~km} \mathrm{~s}^{-1}$. It is interesting to note that $\mathrm{DCO}^{+}$presents an emission peak toward $\mathrm{B} 1 \mathrm{~b}-\mathrm{N}$ at a velocity of $\sim 7.5 \mathrm{~km} \mathrm{~s}^{-1}$ in agreement with the $\mathrm{N}_{2} \mathrm{H}^{+}$and $\mathrm{NH}_{2} \mathrm{D}$ observations by Daniel et al. (2013). Recent interferometric 
A. Fuente et al.: Ionization fraction and the enhanced sulfur chemistry in Barnard 1
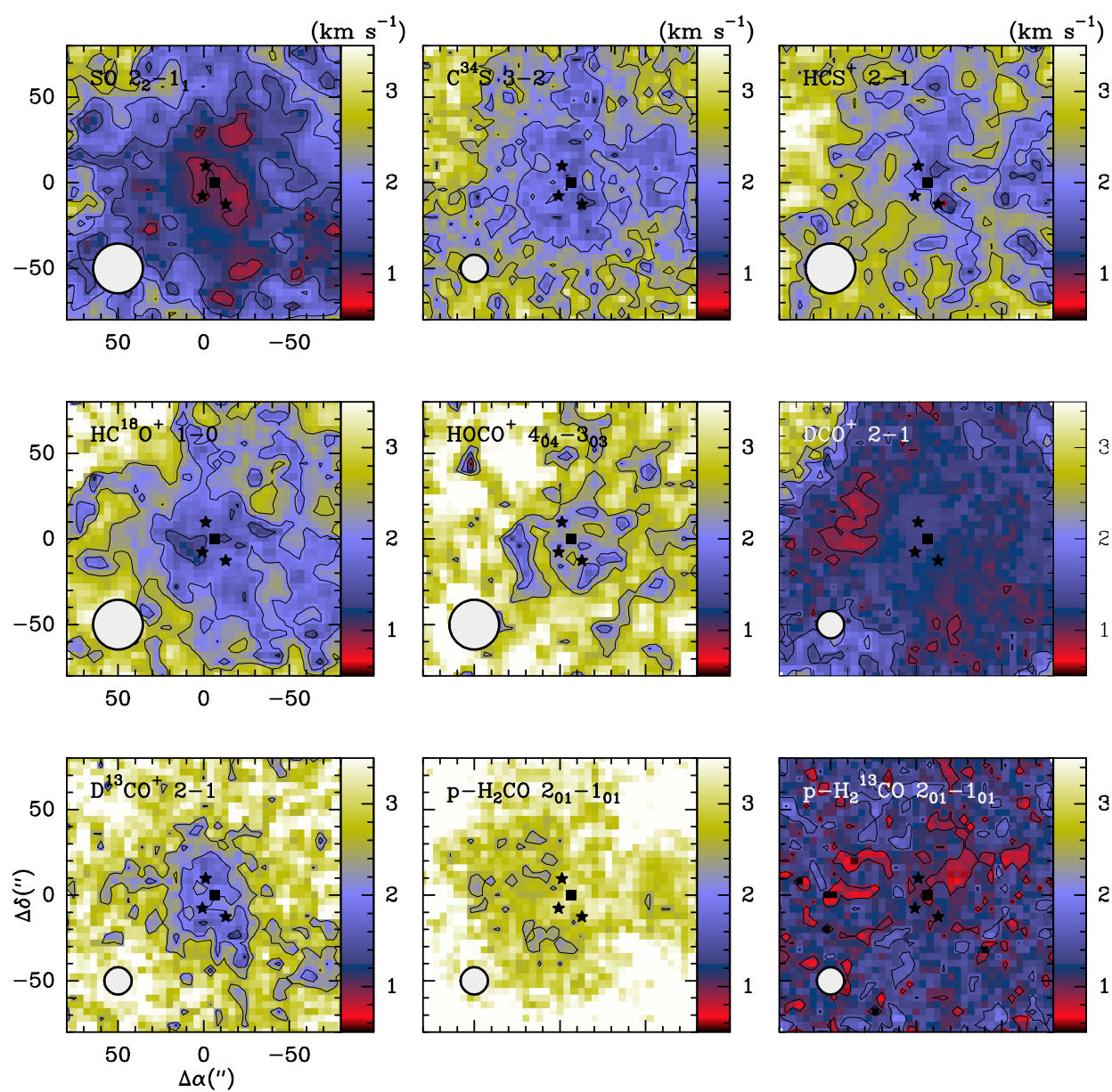

Fig. 3. Maps of the linewidths of the SO $2_{2} \rightarrow 1_{1}, \mathrm{C}^{34} \mathrm{~S} 3 \rightarrow 2$, $\mathrm{HCS}^{+} 2 \rightarrow 1, \mathrm{HC}^{18} \mathrm{O}^{+} 1 \rightarrow 0, \mathrm{HOCO}^{+} 4_{04} \rightarrow 33_{03}, \mathrm{DCO}^{+} 2 \rightarrow 1, \mathrm{D}^{13} \mathrm{CO}^{+} 2 \rightarrow 1$, p- $\mathrm{H}_{2} \mathrm{CO} 2_{02} \rightarrow 2_{01}, \mathrm{p}-\mathrm{H}_{2}^{13} \mathrm{CO} 2_{02} \rightarrow 2_{01}$ lines. Symbols are the same as in Fig. 2.

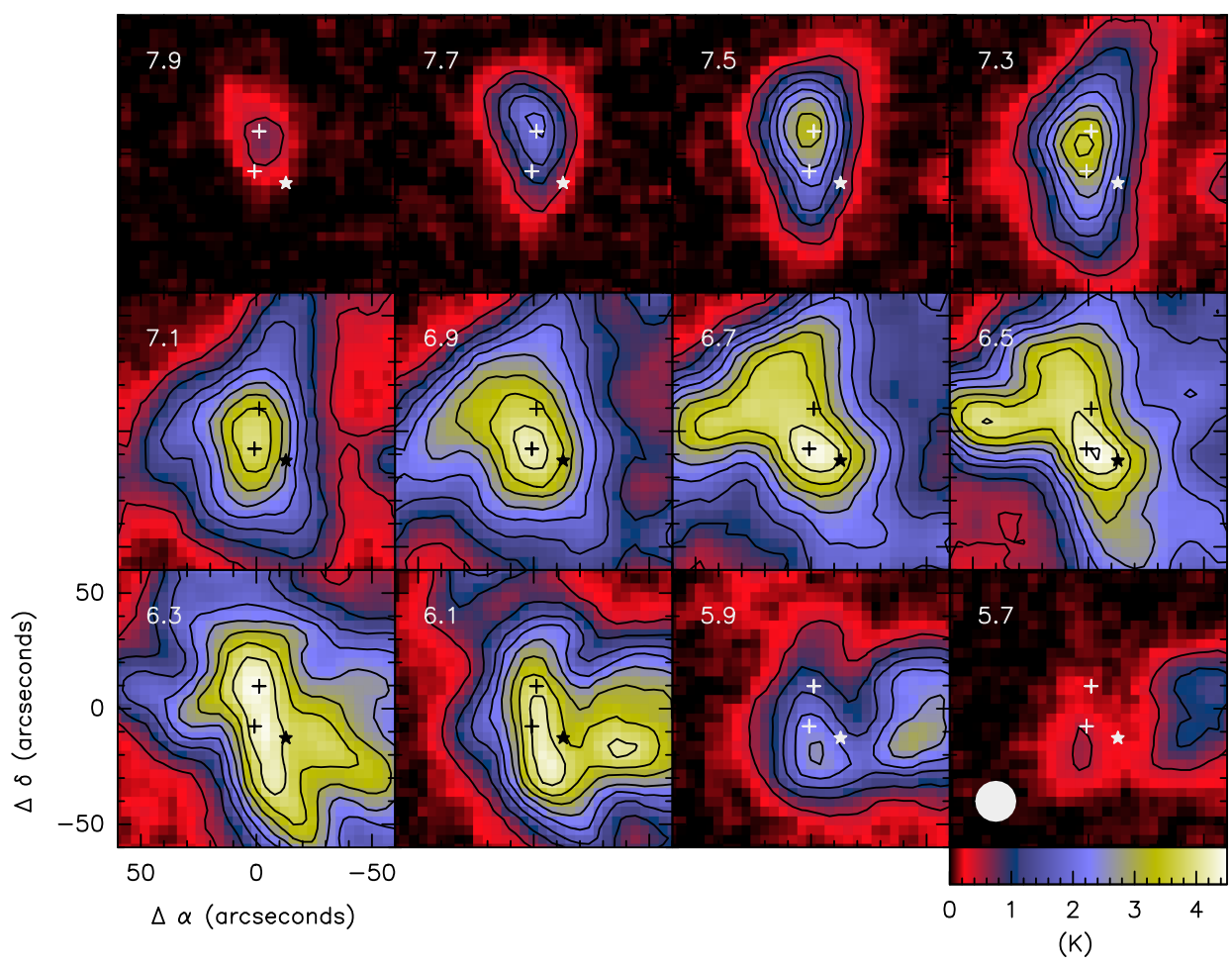

Fig. 4. Spectral maps of the $\mathrm{DCO}^{+} 2 \rightarrow 1$ line as observed with the IRAM 30-m telescope. The central velocity is indicated in the top-left corner. Contours are $0.5 \mathrm{~K}$ to $4.5 \mathrm{~K}$ by steps of $0.5 \mathrm{~K}$. The crosses indicate the positions of the protostellar cores B1b-N and B1b-S. The star denotes the position of B1b-W. In the last panel we plot the beam at $144.277 \mathrm{GHz}$. 


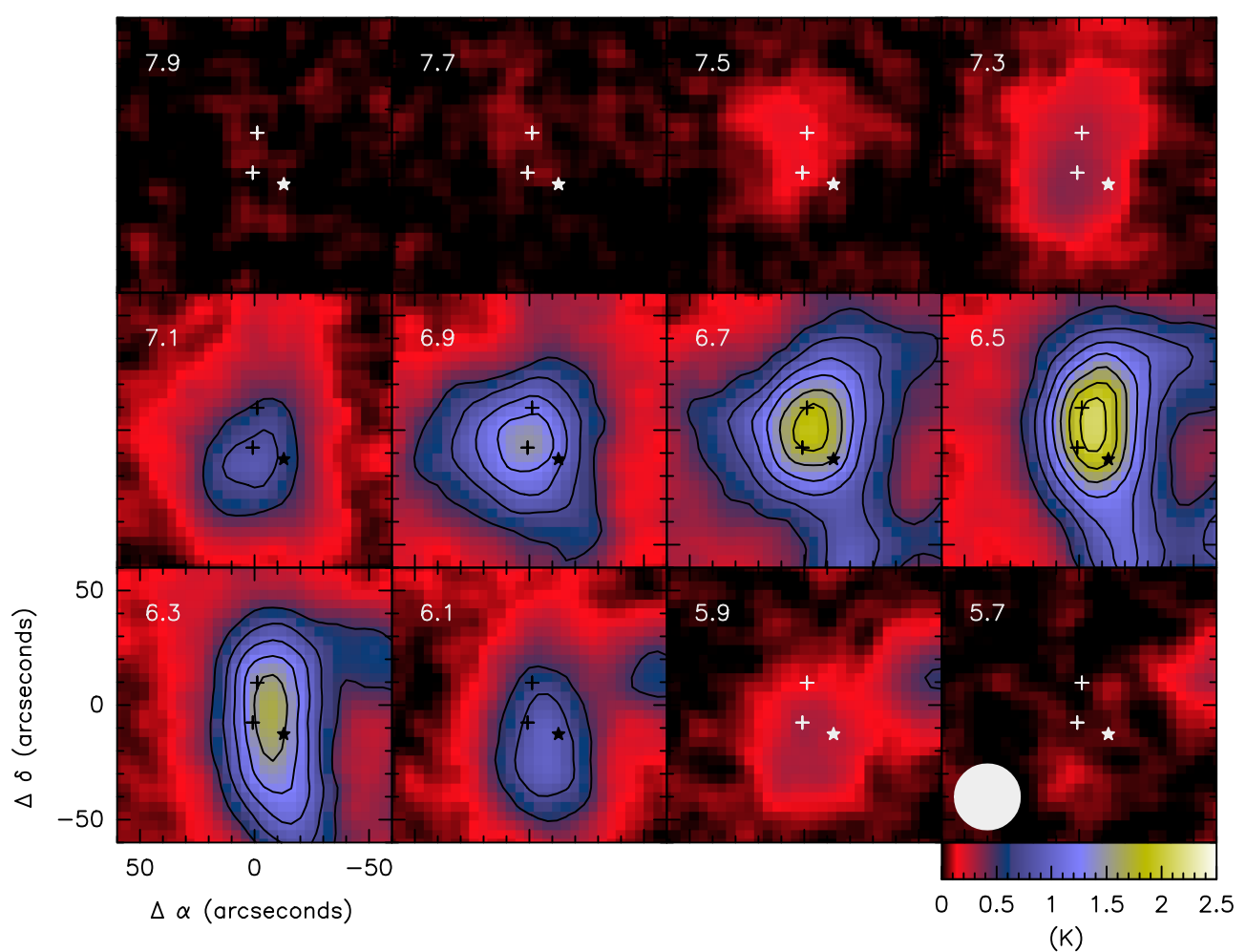

Fig. 5. Spectral maps of the SO $2_{2} \rightarrow 1_{1}$ line as observed with the IRAM 30-m telescope. The central velocity is indicated in the top-left corner. Contours are $0.5 \mathrm{~K}$ to $4.5 \mathrm{~K}$ by steps of $0.25 \mathrm{~K}$. The crosses indicate the positions of the protostellar cores B1b-N and B1b-S. The star denotes the position of B1b-W. In the last panel we plot the beam at $86.094 \mathrm{GHz}$.

observations revealed the kinematical structure of this region at scales of 500-1000 AU (Huang \& Hirano 2013; Gerin et al. 2015). Huang \& Hirano (2013) detected intense emission of the $\mathrm{N}_{2} \mathrm{H}^{+}$and $\mathrm{N}_{2} \mathrm{D}^{+} 3 \rightarrow 2$ lines toward the two protostellar cores B1b-N and B1b-S using the SMA. The $\mathrm{N}_{2} \mathrm{D}^{+}$emission is centered at $\sim 7.0 \pm 0.2 \mathrm{~km} \mathrm{~s}^{-1}$ toward B1b-N and at $\sim 6.5 \pm 0.2 \mathrm{~km} \mathrm{~s}^{-1}$ toward B1b-S, confirming the existence of the two velocity components.

In the cases of $\mathrm{H}_{2}{ }^{13} \mathrm{CO}, \mathrm{H}_{2} \mathrm{~S}, \mathrm{HOCO}^{+}$, and in one transition of CCS, OCS, and $\mathrm{SO}_{2}$ (see Table B.1), we detect wings at redshifted velocities. Compact outflows are detected toward each protostellar core with wings in the 0 to $15 \mathrm{~km} \mathrm{~s}^{-1}$ velocity range (Gerin et al. 2015). In Fig. 6 we overlay the $30 \mathrm{~m}$ beam at $3 \mathrm{~mm}$ and $1 \mathrm{~mm}$ on the interferometric $\mathrm{H}_{2} \mathrm{CO}$ map. The B1b-N outflow and the red wing of the B1b-S outflow lie within our beam at $3 \mathrm{~mm}$, but the whole $\mathrm{B} 1 \mathrm{~b}-\mathrm{N}$ outflow and a fraction of the red lobe of the B1b-S outflow are missed in the $2 \mathrm{~mm}$ and $1 \mathrm{~mm}$ observations. The emission at the outflow velocities is not taken into account in our calculations because we are only interested in the two velocity components associated with the core emission (see Sect. 5 for details).

\section{Column density calculations}

In order to investigate abundance variations between the two velocity components, we carried out a separate analysis for each. The spectral resolution of our observations is different for the $3 \mathrm{~mm}, 2 \mathrm{~mm}$ and $1 \mathrm{~mm}$ bands. To carry out a uniform analysis, we degraded the velocity resolution to a common value of $0.25 \mathrm{~km} \mathrm{~s}^{-1}$ and then fitted Gaussians to each one. In the fitting, we fixed the central velocities to $6.5 \mathrm{~km} \mathrm{~s}^{-1}$ and $\sim 7.0 \mathrm{~km} \mathrm{~s}^{-1}$, and the linewidths to $\Delta v=0.7 \mathrm{~km} \mathrm{~s}^{-1}$ and $1.0 \mathrm{~km} \mathrm{~s}^{-1}$, respectively. The fitting returns the integrated intensity for each component. This procedure gives an excellent fit for most lines and neglects

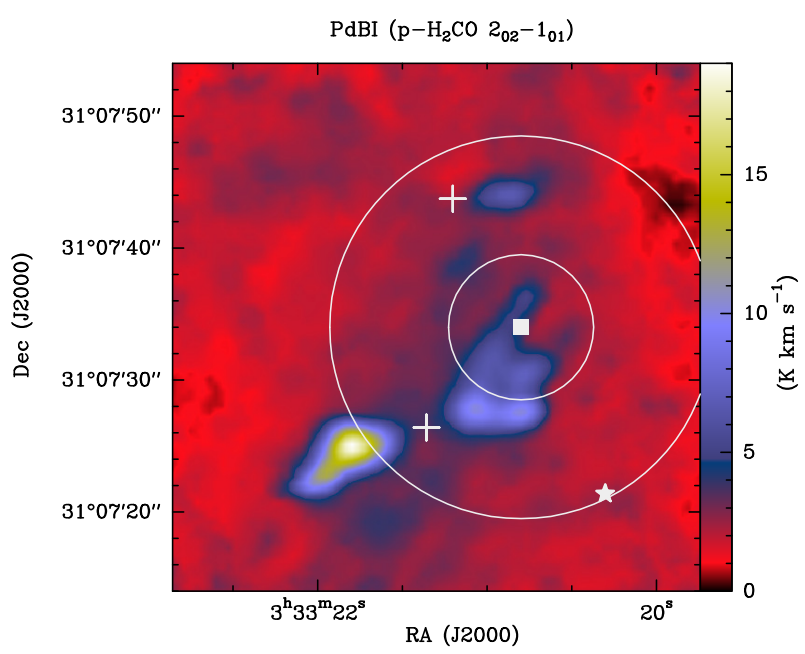

Fig. 6. Interferometric integrated intensity map of the outflows associated with $\mathrm{B} 1 \mathrm{~b}-\mathrm{N}$ and $\mathrm{B} 1 \mathrm{~b}-\mathrm{S}$ in the $\mathrm{H}_{2} \mathrm{CO} 2_{0,2}-1_{0,1}$ line (Gerin et al. 2015). The crosses indicate the positions of the protostellar cores, $\mathrm{B} 1 \mathrm{~b}-\mathrm{N}$ and B1b-S, and the star, of B1b-W. The position targeted by our spectral survey is drawn with a white square and the beams of the $30-\mathrm{m}$ telescope at $3 \mathrm{~mm}$ and $1 \mathrm{~mm}$ are indicated by white circles.

possible line wings with velocities $>7.5 \mathrm{~km} \mathrm{~s}^{-1}$. The final integrated intensities are shown in Table B.1. We are aware that this simple method is not able to cleanly separate the contribution from each component. There is some unavoidable velocity overlap between the two components along the line of sight and our spatial resolution is limited (see Figs. 4 and 5). In spite of that, our simple method help to detect possible chemical gradients.

Several isotopologues of each molecule were detected as expected taking the large extinction in this core into account 
Table 2. Column density calculations.

\begin{tabular}{|c|c|c|c|c|c|c|c|c|}
\hline \multirow[t]{3}{*}{ Molecule } & \multicolumn{5}{|c|}{ LTE } & \multicolumn{3}{|c|}{$\mathrm{LVG}^{1}$} \\
\hline & \multicolumn{2}{|c|}{$6.5 \mathrm{~km} \mathrm{~s}^{-1}$} & \multicolumn{2}{|c|}{$7.0 \mathrm{~km} \mathrm{~s}^{-1}$} & \multirow{2}{*}{$\begin{array}{r}\text { Total } \\
N_{\mathrm{X}}\left(\mathrm{cm}^{-2}\right)\end{array}$} & \multirow{2}{*}{$\begin{array}{c}6.5 \mathrm{~km} \mathrm{~s}^{-1} \\
N_{\mathrm{X}}\left(\mathrm{cm}^{-2}\right) \\
\end{array}$} & \multirow{2}{*}{$\begin{array}{c}7.0 \mathrm{~km} \mathrm{~s}^{-1} \\
N_{\mathrm{X}}\left(\mathrm{cm}^{-2}\right)\end{array}$} & \multirow{2}{*}{$\begin{array}{c}\text { Total } \\
N_{\mathrm{X}}\left(\mathrm{cm}^{-2}\right) \\
\end{array}$} \\
\hline & $\mathrm{ot}(\mathrm{K})$ & $N_{\mathrm{X}}\left(\mathrm{cm}^{-2}\right)$ & $T_{\mathrm{rot}}(\mathrm{K})$ & $N_{\mathrm{X}}\left(\mathrm{cm}^{-2}\right)$ & & & & \\
\hline \multicolumn{6}{|c|}{ Ions } & \multicolumn{3}{|c|}{ Ions } \\
\hline $\mathrm{HC}^{18} \mathrm{O}^{+}$ & & $1.7_{-0.1}^{+0.1} \times 10^{11}$ & $4.2_{-0.7}^{+0.4}$ & $1.5_{-}^{+}$ & $3.2 \times 10^{11}$ & $2.0_{-0.1}^{+0.1} \times 10^{11}$ & $1.4_{-0.3}^{+0.2} \times 10^{11}$ & $3.4 \times 10^{11}$ \\
\hline${ }^{13} \mathrm{CO}^{+}$ & & $1.2_{-0.2}^{-0.1} \times 10^{11}$ & $4.5_{-0.2}^{+0.2}$ & $3.9_{-0.5}^{+0.5} \times 10^{11}$ & $5.1 \times 10^{11}$ & $2.0_{-0.1}^{+0.1} \times 10^{11}$ & $4.5_{-0.1}^{+0.1} \times 10^{11}$ & $6.5 \times 10^{11}$ \\
\hline $\mathrm{HOCO}^{+}$ & $11.9_{-0.3}^{+0.3}$ & $5.2_{-0.3}^{-0.2} \times 10^{11}$ & $10.7_{-1.0}^{+1.2}$ & $2.4_{-0.7}^{+1.0} \times 10^{11}$ & $7.6 \times 10^{11}$ & $5.2_{-0.2}^{+0.2} \times 10^{11}$ & $3.0_{-0.2}^{+0.1} \times 10^{11}$ & $8.2 \times 10^{11}$ \\
\hline $\mathrm{DOCO}^{+}$ & $11^{a}$ & $7.0_{-0.9}^{+0.9} \times 10^{10}$ & $11^{a}$ & $4.0_{-2.0}^{+2.0} \times 10^{10}$ & $1.1 \times 10^{11}$ & $9.0_{-1.1}^{+1.1} \times 10^{10}$ & $5.0_{-2.0}^{+2.0} \times 10^{10}$ & $1.1 \times 10^{11}$ \\
\hline $\mathrm{SO}^{+}$ & 0.8 .8 & $7.6_{-1.6}^{+2.0} \times 10^{11}$ & $8.3_{-1.4}^{+2.2}$ & $4.8_{-2.2}^{+4.1} \times 10^{11}$ & $1.2 \times 10^{12}$ & & & \\
\hline $\mathrm{HCS}^{+}$ & $10.6_{-3.0}^{+6.5}$ & $2.1_{-1.3}^{+3.6} \times 10^{11}$ & $7.5_{-0.6}^{+0.5}$ & $6.2_{-0.2}^{+0.2} \times 10^{11}$ & $8.3 \times 1$ & $5.5_{-0.1}^{+0.1} \times 10^{11}$ & $5.5_{-0.1}^{+0.1} \times 10^{11}$ & $1.1 \times 10^{12}$ \\
\hline DC & $10^{b}$ & $5.0_{-1.0}^{+1.0} \times 10^{10}$ & & & $5.0 \times 1$ & $4.5_{-1.1}^{+1.1 .1} \times 10^{10}$ & & $4.5>$ \\
\hline $\mathrm{HCNH}^{+}$ & $29.0_{-12}^{+50}$ & $5.1_{-0.7}^{+4.1} \times 10^{12}$ & $8.9_{-2.9}^{+5.3}$ & $3.8_{-0.5}^{+1.5} \times 10^{12}$ & $8.9 \times 10^{12}$ & $3.7_{-0.4}^{+0.4} \times 10^{12}$ & $4.0_{-0.4}^{+0.4} \times 10^{12}$ & $7.7 \times 10^{12}$ \\
\hline $\mathrm{H}_{2}$ & & & $10^{b}$ & $2.0_{-1.0}^{+1.0} \times 10^{11}$ & $2.0_{-1.0}^{+1.0} \times 10^{11}$ & & & \\
\hline $\mathrm{CO}$ & & & $10^{b}$ & $<6.0$ & $<6$. & & & \\
\hline & & & $10^{b}$ & $<2.0\rangle$ & & & & \\
\hline \multicolumn{6}{|c|}{ Neutrals } & \multicolumn{3}{|c|}{ Neutrals } \\
\hline${ }^{13} \mathrm{C}^{18} \mathrm{O}$ & $5.5_{-0.9}^{+1.3}$ & $2.4_{-0.2}^{+0.2} \times 10^{13}$ & $10.9_{-1.4}^{+0.9}$ & $5.2^{+}$ & $7.6 \times$ & $3.0_{-0.2}^{+0.2} \times 10^{13}$ & $6.0_{-0.3}^{+0.3} \times 10^{13}$ & 9.0 \\
\hline $\mathrm{o}-\mathrm{H}_{2}{ }^{13} \mathrm{CO}$ & & $6.1_{-1.5}^{+2.2} \times 10^{11}$ & $5.2_{-0.3}^{+0.9}$ & $4.9_{-1.9}^{+3.2} \times 10^{11}$ & $1.1 \times$ & $1.1_{-0.6}^{-0.2} \times 10^{12}$ & $1.3_{-0.7}^{-0.3} \times 10^{12}$ & $2.4 \times 10^{12}$ \\
\hline${ }^{13} \mathrm{CS}$ & $7.1_{-1.1}^{+1.6}$ & $7.8_{-5.7}^{-2.0} \times 10^{11}$ & $3.2_{-0.7}^{+0.3}$ & $3.1_{-1.6}^{-1.9} \times$ & $3.9 \times 10^{12}$ & $1.3_{-0.1}^{+0.0 .1} \times 10^{12}$ & $9.0_{-0.3}^{+0.3} \times 10^{11}$ & $2.2 \times 10^{12}$ \\
\hline $\mathrm{S}^{18} \mathrm{O}$ & $6.8_{-0.6}^{+0.1}$ & $1.6_{-0.5}^{+0.6} \times 10^{12}$ & $8.2_{-1.4}^{+2.0}$ & $0.6_{-0.3}^{+0.0} \times 10^{12}$ & $2.3 \times 10^{12}$ & $2.2_{-0.1}^{+0.1} \times 10^{12}$ & $8.0_{-0.3}^{+0.3} \times 10^{11}$ & $3.0 \times 10^{12}$ \\
\hline${ }^{3} \mathrm{SO}$ & $12.3_{-4.0}^{+0.3}$ & $1.6_{-0.6}^{+1.0} \times 10^{12}$ & $18.9_{-8.3}^{+74}$ & $0.7_{-0.4}^{-0.7} \times 10^{12}$ & $2.3 \times 10^{12}$ & $3.8_{-0.1}^{-0.1} \times 10^{12}$ & $6.0_{-2.0}^{-2.0} \times 10^{11}$ & $4.4 \times 10^{12}$ \\
\hline${ }^{34} \mathrm{SO}_{2}$ & $5.8_{-1.2}^{+2.0}$ & $1.1_{-0.5}^{+1.0} \times 10^{12}$ & & & & $1.1_{-0.1}^{+0.1} \times 10^{12}$ & & \\
\hline $\mathrm{SO}_{2}$ & $10.1_{-1.0}^{+1.0}$ & $7.9_{-2.0}^{+3.0} \times 10^{12}$ & $10.2_{-1.2}^{+2.0}$ & $2.9_{-0.9}^{+1.3} \times 10^{12}$ & $1.1 \times 10^{13}$ & $1.6_{-0.1}^{+0.1} \times 10^{13}$ & $4.5_{-0.1}^{+0.1} \times 10^{12}$ & $2.0 \times 10^{13}$ \\
\hline $\mathrm{OC}^{34} \mathrm{~S}$ & $8.7_{-0.5}^{+0.6}$ & $1.2_{-0.2}^{+0.2} \times 10^{12}$ & & & & $1.0_{-0.3}^{+0.3} \times 10^{12}$ & & \\
\hline OCS & $11.7_{-4.0}^{+13.0}$ & $1.5_{-0.6}^{+0.9} \times 10^{13}$ & $20.4_{-1.3}^{+1.4}$ & $4.9_{-0.6}^{+0.6} \times 10^{12}$ & $1.9 \times 10^{13}$ & $1.7_{-0.1}^{+0.1} \times 10^{13}$ & $8.0_{-4.0}^{+4.0} \times 10^{11}$ & $1.7 \times 10^{13}$ \\
\hline $\mathrm{H}_{2}{ }^{13} \mathrm{CS}$ & 7.4 & & & & & & & \\
\hline $\mathrm{CCS}$ & $8.3_{-0.6}^{+0.7}$ & $5.9_{-1.7}^{+2.3} \times 10^{12}$ & $8.2_{-0.6}^{+0.7}$ & $3.6_{-1.0}^{+1.3} \times 10^{12}$ & $9.5 \times 10^{12}$ & $9.3_{-0.1}^{+0.1} \times 10^{12}$ & $5.2_{-0.1}^{+0.1} \times 10^{12}$ & $1.4 \times 10^{13}$ \\
\hline $\mathrm{CC}^{34} \mathrm{~S}$ & & & & & & $3.5_{-0.7}^{+0.7} \times 10^{11}$ & $2.5_{-0.7}^{+0.7} \times 10^{11}$ & $6.0 \times 10^{11}$ \\
\hline CCCS & $8.6_{-1.2}^{+1.6}$ & & & & $2.2 \times 10^{12}$ & & & \\
\hline $\mathrm{o}-\mathrm{H}_{2} \mathrm{~S}$ & $10^{d}$ & $7.0_{-0.2}^{+0.2} \times 10^{12}$ & 10 & $3.0_{-0.2}^{+0.2} \times 10^{12}$ & $1.0 \times 10^{13}$ & & & \\
\hline NS & $10^{d}$ & $5.4_{-0.2}^{-0.2} \times 10^{12}$ & 10 & $9.0_{-0.2}^{+0.2} \times 10^{12}$ & $1.4 \times 10^{13}$ & & & \\
\hline
\end{tabular}

Notes. ${ }^{(1)}$ LVG calculations assuming $T_{k}=12 \mathrm{~K}$ and $n\left(\mathrm{H}_{2}\right)=10^{5} \mathrm{~cm}^{-3}$; ${ }^{(a)}$ assuming the rotation temperature of $\mathrm{HOCO}^{+}$; ${ }^{(b)}$ assuming the rotation temperature of $\mathrm{HCS}^{+}{ }^{(c)}$ in this case the ortho and para species are treated together (ortho-to-para $(\mathrm{OTP})=3$ ) and we assumed the rotation temperature calculated for $\mathrm{H}_{2} \mathrm{CS}$ by Marcelino et al. (2005); ${ }^{(d)}$ reasonable guess for the rotation temperature.

( $A_{v} \sim 76 \mathrm{mag}$ ). As zero-order approximation, we derived the column densities and fractional abundances of the different species using the rotation diagram technique. This technique gives a good estimate of the total number of molecules within our beam as far as the observed lines are optically thin. To be sure of this requirement, we selected the rarest isotopologue of each species. Furthermore, we assumed that the emission is filling the beam. To calculate the column densities and abundances of the main isotopologues, we adopted ${ }^{12} \mathrm{C} /{ }^{13} \mathrm{C}=60,{ }^{16} \mathrm{O} /{ }^{18} \mathrm{O}=550$, ${ }^{32} \mathrm{~S} /{ }^{34} \mathrm{~S}=22.5$, and ${ }^{34} \mathrm{~S} /{ }^{33} \mathrm{~S}=6$ (Chin et al. 1996; Wilson et al. 1999).

In Table 2 we show the results of our rotational diagram calculations. The errors in the rotation temperatures and column densities are those derived from the least squares fitting. There are, however, other kind of uncertainties that are not considered in these numbers. One source of uncertainty is the unknown beam filling factor. Since the beam is dependent on the frequency, this could bias the derived rotation temperature. To estimate the error from this uncertainty, we calculated the column densities with an alternative method. Daniel et al. (2013) modeled the density structure of the $\mathrm{B} 1 \mathrm{~b}$ core on the basis of dust continuum at $350 \mu \mathrm{m}$ and $1.2 \mathrm{~mm}$. These authors obtained that the mean density in the $\sim 29^{\prime \prime}$ beam is $n\left(\mathrm{H}_{2}\right) \approx 10^{5} \mathrm{~cm}^{-3}$. Lis et al. (2010) derived a gas kinetic temperature of $T_{K}=12 \mathrm{~K}$ from $\mathrm{NH}_{3}$ observations. We adopted these physical conditions to derive the molecular column densities by fitting the integrated intensity of the lowest energy transition using the LVG code MADEX (Cernicharo 2012). The results are shown in Table 2. Column densities derived using the rotation diagram technique and LVG calculations agree within a factor of 2 for all the compounds. We consider that our estimates are accurate within this factor.

In Table 3 we show the fractional abundances of the studied species and some interesting molecular abundance ratios. To estimate the fractional abundances for each velocity component, we assumed that the abundance of ${ }^{13} \mathrm{C}^{18} \mathrm{O}$ is the same 
Table 3. Fractional abundances and abundance ratios.

\begin{tabular}{|c|c|c|c|c|c|c|}
\hline \multirow[t]{2}{*}{ Molecule } & \multicolumn{3}{|c|}{ LTE } & \multicolumn{3}{|c|}{$\mathrm{LVG}^{1}$} \\
\hline & $6.5 \mathrm{~km} \mathrm{~s}^{-1}$ & $7.0 \mathrm{~km} \mathrm{~s}^{-1}$ & Total & $6.5 \mathrm{~km} \mathrm{~s}^{-1}$ & $7.0 \mathrm{~km} \mathrm{~s}^{-1}$ & Total \\
\hline \multicolumn{4}{|c|}{ Ions } & \multicolumn{3}{|c|}{ Ions } \\
\hline$X\left(\mathrm{HC}^{18} \mathrm{O}^{+}\right) \times 550$ & $3.9 \times 10^{-9}$ & $1.8 \times 10^{-9}$ & $2.5 \times 10^{-9}$ & $4.6 \times 10^{-9}$ & $1.7 \times 10^{-9}$ & $2.7 \times 10^{-9}$ \\
\hline$X\left(\mathrm{D}^{13} \mathrm{CO}^{+}\right) \times 60$ & $2.7 \times 10^{-10}$ & $4.7 \times 10^{-10}$ & $4.0 \times 10^{-10}$ & $4.6 \times 10^{-10}$ & $5.4 \times 10^{-10}$ & $5.1 \times 10^{-10}$ \\
\hline$X\left(\mathrm{HOCO}^{+}\right)$ & $2.0 \times 10^{-11}$ & $4.8 \times 10^{-12}$ & $1.0 \times 10^{-11}$ & $2.0 \times 10^{-11}$ & $6.0 \times 10^{-12}$ & $1.1 \times 10^{-11}$ \\
\hline$X\left(\mathrm{DOCO}^{+}\right)$ & $2.9 \times 10^{-12}$ & $8.0 \times 10^{-13}$ & $1.4 \times 10^{-12}$ & $3.6 \times 10^{-12}$ & $1.0 \times 10^{-12}$ & $1.4 \times 10^{-12}$ \\
\hline$X\left(\mathrm{SO}^{+}\right)$ & $3.1 \times 10^{-11}$ & $9.6 \times 10^{-12}$ & $1.6 \times 10^{-11}$ & & & \\
\hline$X\left(\mathrm{HCS}^{+}\right)$ & $8.1 \times 10^{-12}$ & $1.2 \times 10^{-11}$ & $1.1 \times 10^{-11}$ & $2.1 \times 10^{-11}$ & $1.1 \times 10^{-11}$ & $1.4 \times 10^{-11}$ \\
\hline$X\left(\mathrm{DCS}^{+}\right)$ & $1.9 \times 10^{-12}$ & & $6.6 \times 10^{-13}$ & $1.7 \times 10^{-12}$ & & $5.9 \times 10^{-13}$ \\
\hline$X\left(\mathrm{HCNH}^{+}\right)$ & $2.0 \times 10^{-10}$ & $7.6 \times 10^{-11}$ & $1.2 \times 10^{-10}$ & $1.4 \times 10^{-10}$ & $8.0 \times 10^{-11}$ & $1.0 \times 10^{-10}$ \\
\hline$X\left(\mathrm{H}_{2} \mathrm{COH}^{+}\right)$ & & $4.0 \times 10^{-12}$ & $2.6 \times 10^{-12}$ & & & \\
\hline$X\left(\mathrm{CO}^{+}\right)$ & & & $<7.9 \times 10^{-13}$ & & & \\
\hline$X\left(\mathrm{HOC}^{+}\right)$ & & & $<2.6 \times 10^{-13}$ & & & \\
\hline$X\left(\mathrm{NNH}^{+}\right)$ & & & $1.4 \times 10^{-9 *}$ & & & \\
\hline$X\left(\mathrm{NND}^{+}\right)$ & & & $5.0 \times 10^{-10 *}$ & & & \\
\hline \multicolumn{4}{|c|}{ Neutrals } & \multicolumn{3}{|c|}{ Neutrals } \\
\hline$X\left({ }^{13} \mathrm{C}^{18} \mathrm{O}\right)$ & $1.0 \times 10^{-9}$ & $1.0 \times 10^{-9}$ & $1.0 \times 10^{-9}$ & $1.2 \times 10^{-9}$ & $1.2 \times 10^{-9}$ & $1.2 \times 10^{-9}$ \\
\hline$X\left(\mathrm{H}_{2}{ }^{13} \mathrm{CO}\right) \times 60^{a}$ & $1.9 \times 10^{-9}$ & $7.8 \times 10^{-10}$ & $1.1 \times 10^{-9}$ & $3.4 \times 10^{-9}$ & $2.1 \times 10^{-9}$ & $2.5 \times 10^{-9}$ \\
\hline$X\left({ }^{13} \mathrm{CS}\right) \times 60$ & $1.8 \times 10^{-9}$ & $3.7 \times 10^{-9}$ & $3.1 \times 10^{-9}$ & $3.0 \times 10^{-9}$ & $1.1 \times 10^{-9}$ & $1.7 \times 10^{-9}$ \\
\hline$X\left(\mathrm{~S}^{18} \mathrm{O}\right) \times 550$ & $3.7 \times 10^{-8}$ & $6.3 \times 10^{-9}$ & $1.8 \times 10^{-8}$ & $5.0 \times 10^{-8}$ & $9.0 \times 10^{-9}$ & $2.2 \times 10^{-8}$ \\
\hline$X\left({ }^{33} \mathrm{SO}\right) \times 135$ & $9.0 \times 10^{-9}$ & $1.8 \times 10^{-9}$ & $4.0 \times 10^{-9}$ & $1.9 \times 10^{-8}$ & $1.6 \times 10^{-9}$ & $7.9 \times 10^{-9}$ \\
\hline$X\left({ }^{34} \mathrm{SO}_{2}\right) \times 22.5$ & $1.0 \times 10^{-9}$ & & & $1.0 \times 10^{-9}$ & & \\
\hline$X\left(\mathrm{SO}_{2}\right)$ & $3.3 \times 10^{-10}$ & $5.6 \times 10^{-11}$ & $1.4 \times 10^{-10}$ & $6.7 \times 10^{-10}$ & $9.0 \times 10^{-11}$ & $2.6 \times 10^{-10}$ \\
\hline$X\left(\mathrm{OC}^{34} \mathrm{~S}\right) \times 22.5$ & $1.1 \times 10^{-9}$ & & & $9.4 \times 10^{-10}$ & & \\
\hline$X(\mathrm{OCS})$ & $6.2 \times 10^{-10}$ & $9.4 \times 10^{-11}$ & $2.4 \times 10^{-10}$ & $7.0 \times 10^{-10}$ & $1.5 \times 10^{-11}$ & $2.2 \times 10^{-10}$ \\
\hline$X\left(\mathrm{H}_{2}{ }^{13} \mathrm{CS}\right) \times 60$ & $4.0 \times 10^{-10}$ & & & & & \\
\hline$X(\mathrm{CCS})$ & $2.4 \times 10^{-10}$ & $6.9 \times 10^{-11}$ & $1.2 \times 10^{-10}$ & $3.9 \times 10^{-10}$ & $1.0 \times 10^{-10}$ & $1.8 \times 10^{-10}$ \\
\hline$X\left(\mathrm{H}_{2} \mathrm{~S}\right)^{a}$ & $3.9 \times 10^{-10}$ & $7.7 \times 10^{-11}$ & $1.7 \times 10^{-10}$ & & & \\
\hline$X(\mathrm{NS})$ & $2.2 \times 10^{-10}$ & $1.7 \times 10^{-10}$ & $1.8 \times 10^{-10}$ & & & \\
\hline$X(\mathrm{HCN})$ & & & $1.2 \times 10^{-8 *}$ & & & \\
\hline \multicolumn{4}{|c|}{ Ion/Neutral and Ion/Ion } & \multicolumn{3}{|c|}{ Ion/Neutral and Ion/Ion } \\
\hline$\frac{N\left(\mathrm{HCO}^{+}\right)}{N(\mathrm{CO})}$ & $1.1 \times 10^{-4}$ & $4.8 \times 10^{-5}$ & $6.9 \times 10^{-5}$ & $1.1 \times 10^{-4}$ & $4.3 \times 10^{-5}$ & $6.8 \times 10^{-5}$ \\
\hline$\frac{N\left(\mathrm{DCO}^{+}\right)}{N\left(\mathrm{HCO}^{+}\right)}$ & 0.07 & 0.26 & 0.15 & 0.1 & 0.32 & 0.19 \\
\hline$\frac{N\left(\mathrm{DCO}^{+}\right)}{N(\mathrm{CO})}$ & $9.0 \times 10^{-6}$ & $1.2 \times 10^{-5}$ & $1.1 \times 10^{-5}$ & $1.1 \times 10^{-5}$ & $1.4 \times 10^{-5}$ & $1.2 \times 10^{-5}$ \\
\hline$\frac{N\left(\mathrm{HOCO}^{+}\right)}{N\left(\mathrm{HCO}^{+}\right)}$ & 0.005 & 0.003 & 0.004 & 0.004 & 0.003 & 0.004 \\
\hline$\frac{N\left(\mathrm{SO}^{+}\right)}{N(\mathrm{SO})}$ & $8.0 \times 10^{-4}$ & 0.0014 & $9.5 \times 10^{-4}$ & & & \\
\hline$\frac{N\left(\mathrm{HCS}^{+}\right)}{N(\mathrm{CS})}$ & 0.004 & 0.003 & 0.0035 & 0.007 & 0.01 & 0.008 \\
\hline$\frac{N\left(\mathrm{DCS}^{+}\right)}{N\left(\mathrm{HCS}^{+}\right)}$ & 0.24 & & 0.06 & 0.08 & & 0.04 \\
\hline$\frac{N\left(\mathrm{HCS}^{+}\right)}{N\left(\mathrm{HCO}^{+}\right)}$ & 0.003 & 0.007 & 0.004 & 0.004 & 0.006 & 0.005 \\
\hline$\frac{N\left(\mathrm{HCNH}^{+}\right)}{N(\mathrm{HCN})}$ & & & 0.01 & & & 0.008 \\
\hline$\frac{N\left(\mathrm{H}_{2} \mathrm{COH}\right)}{N\left(\mathrm{H}_{2} \mathrm{CO}\right)}$ & & 0.005 & 0.002 & & & \\
\hline$\frac{N\left(\mathrm{CO}^{+}\right)}{N\left(\mathrm{HCO}^{+}\right)}$ & & & $<3 \times 10^{-4}$ & & & \\
\hline$\frac{N\left(\mathrm{HOC}^{+}\right)}{N\left(\mathrm{HCO}^{+}\right)}$ & & & $<1 \times 10^{-4}$ & & & \\
\hline \multicolumn{4}{|c|}{ Neutral/Neutral } & \multicolumn{3}{|c|}{ Neutral/Neutral } \\
\hline$\frac{N(\mathrm{SO})}{N(\mathrm{CS})}$ & 19 & 1.8 & 5 & 17 & 8 & 13 \\
\hline$\frac{N\left(\mathrm{SO}_{2}\right)}{N(\mathrm{SO})}$ & 0.03 & 0.008 & 0.008 & 0.01 & 0.009 & 0.01 \\
\hline$\frac{N(\mathrm{CCS})}{N(\mathrm{CS})}$ & 0.13 & 0.02 & 0.04 & 0.12 & 0.09 & 0.11 \\
\hline$\frac{N\left(\mathrm{H}_{2} \mathrm{CS}\right)}{N(\mathrm{CS})}$ & 0.20 & & & & & \\
\hline$\frac{N(\mathrm{OCS})}{N(\mathrm{CS})}$ & 0.57 & 0.03 & 0.08 & 0.22 & 0.01 & 0.13 \\
\hline
\end{tabular}

Notes. ${ }^{(1)}$ LVG calculations assuming $T_{\mathrm{k}}=12 \mathrm{~K}$ and $n\left(\mathrm{H}_{2}\right)=10^{5} \mathrm{~cm}^{-3}$. Numbers in boldface are used in Fig. B.2 to Fig. B.6 to compare with observations. ${ }^{(*)}$ From Daniel et al. (2013); ${ }^{(a)}$ From Table 2 assuming OTP $=3$. 
for the two components and adopted a total molecular hydrogen column density of $N\left(\mathrm{H}_{2}\right)=7.6 \times 10^{22} \mathrm{~cm}^{-2}$ (Daniel et al. 2013). Most of the fractional abundances and abundance ratios agree within the uncertainties for the two velocity components. There are, however, significant chemical differences for some species. One difference is that the $\mathrm{HCO}^{+}$deuterium fraction is a factor $\sim 3$ higher in the $7 \mathrm{~km} \mathrm{~s}^{-1}$ component than in the $6.5 \mathrm{~km} \mathrm{~s}^{-1}$ one. The spectral velocity maps of the $\mathrm{DCO}^{+} 2 \rightarrow 1$ and $\mathrm{D}^{13} \mathrm{CO}^{+} 2 \rightarrow 1$ lines show that the $7 \mathrm{~km} \mathrm{~s}^{-1}$ component is associated with the cold protostar B1b-N. The second difference is that the SO/CS abundance ratio is $2-10$ times larger in the $\sim 6.5 \mathrm{~km} \mathrm{~s}^{-1}$ component than in the $\sim 7.0 \mathrm{~km} \mathrm{~s}^{-1}$ component. High SO abundances, up to $\sim 10^{-7}$, are found in bipolar outflows because of the release of sulfuretted compounds into the gas phase (Bachiller \& Pérez Gutiérrez 1997). One could think that the high SO abundances could then result from the interaction of the cold core with the B1b-S outflow, or, more likely, of the interaction of the dense core with the B1a and/or B1d outflows. However, the profiles of the SO lines are very narrow, which is contrary to one would expect if the emission were coming from a shock or a turbulent medium. In Sect. 6, we discuss in detail the sulfur chemistry and possible explanations for the high SO abundance in Barnard 1.

One important result is that the $\left[\mathrm{XH}^{+}\right] /[\mathrm{X}]$ ratios do not present variations between the two velocity components. This supports our interpretation that the ionization fraction is mainly driven by $\mathrm{CR}$ and is consistent with the measured $\mathrm{H}_{2}$ column density $\left(7.6 \times 10^{22} \mathrm{~cm}^{-2}\right)$. The core is expected to be thoroughly permeable to $\mathrm{CR}$ and the ionization does not present significant fluctuations across the envelope.

\section{Chemical modeling}

We perform chemical modeling to constrain the physical and chemical properties in B1b. We do not include here the accretion/desorption mechanisms of gas-phase molecules on the grains and instead vary the amount of elemental abundances of heavy atoms available in the gas phase to mimic these effects. We explicitly consider, however, the formation on grains of the molecular hydrogen, $\mathrm{H}_{2}$, and the isotopic substituted forms HD and $\mathrm{D}_{2}$. Such an approach, although primitive, allows us to disentangle the main gas-phase processes at work. We use both a steady-state and time-dependent model to explore the chemistry and verified that the steady-state results are recovered by the time-dependent code for sufficiently large evolution time (typically $10 \mathrm{Myr}$ ).

Our chemical network contains 261 species linked through more than 5636 chemical reactions. Ortho and para forms of $\mathrm{H}_{2}$, $\mathrm{H}_{2}^{+}, \mathrm{H}_{3}^{+}, \mathrm{D}_{2}, \mathrm{H}_{2} \mathrm{D}^{+}, \mathrm{D}_{2} \mathrm{H}^{+}$, and $\mathrm{D}_{3}^{+}$are explicitly introduced, following the pioneering papers of Flower et al. $(2004,2006)$ for heavily depleted regions, and including the chemistry of carbon, oxygen, nitrogen, and sulfur. Discriminating between para and ortho forms of $\mathrm{H}_{2}$ is particularly critical for deuteration in cold regions via the $\mathrm{H}_{3}^{+}+\mathrm{HD} \leftrightarrow \mathrm{H}_{2} \mathrm{D}^{+}+\mathrm{H}_{2}$ reaction as the endothermicity of the reverse reaction is of the same order of magnitude as the rotational energy of $\mathrm{H}_{2}$, as first emphasized by Pagani et al. (1992). Nitrogen chemistry is also very sensitive to the ortho and para forms of $\mathrm{H}_{2}$, as pointed out by Le Bourlot (1991), because of the small endoergicity of the $\mathrm{N}^{+}+\mathrm{H}_{2} \rightarrow$ $\mathrm{NH}^{+}+\mathrm{H}$ reaction. Whereas a definitive understanding of this reaction is still pending (Zymak et al. 2013), we follow the prescription of Dislaire et al. (2012) for the reaction rate coefficients of $\mathrm{N}^{+}+\mathrm{H}_{2}(\mathrm{p} / \mathrm{o})$ reaction. Our chemical network also contains the recent updates coming from experimental studies of reactions involving neutral nitrogen atoms as reviewed in Wakelam et al. (2013). We also introduced species containing two nitrogen atoms, such as $\mathrm{C}_{2} \mathrm{~N}_{2}$ and $\mathrm{C}_{2} \mathrm{~N}_{2} \mathrm{H}^{+}$, following the recent detection of $\mathrm{C}_{2} \mathrm{~N}_{2} \mathrm{H}^{+}$by Agúndez et al. (2015) for which the reaction rate coefficients have essentially been taken from the UMIST astrochemical database (McElroy et al. 2013). An important feature that is not fully recognized is the link between sulfur and nitrogen chemistry as shown by the detection of NS, which is found both in regions of massive star formation and in cold dark clouds (McGonagle et al. 1994; McGonagle \& Irvine 1997). The main formation reaction is through $\mathrm{S}+\mathrm{NH}$ whose rate is given as $10^{-10} \mathrm{~cm}^{3} \mathrm{~s}^{-1}$ in astrochemical databases, which then also induces that sulfur chemistry may depend on $\mathrm{o} / \mathrm{p}$ ratio of $\mathrm{H}_{2}$.

Our methodology is the following: We run the timedependent model for a set of standard elemental abundances and cosmic ray ionization rates to determine which molecular abundances and molecular abundance ratios reach the steady state in a few 0.1-1 Myr, which is the estimated age for B1b. These molecular abundances and abundance ratios are expected to be in equilibrium and are used to explore the parameter space using steady-state calculations. On basis of the steady-state calculations, we select the most plausible values and run the timedependent model to confirm that our fit is correct. In all our calculations, we assume uniform physical conditions and fix $n\left(\mathrm{H}_{2}\right)=10^{5} \mathrm{~cm}^{-3}$ and $T_{k}=12 \mathrm{~K}$, according with previous works (Lis et al. 2010; Daniel et al. 2013). We iterate several times to get the best match with the observations. The predictions of our best-fit model are compared with observations in Figs. B.2 and B.3.

\subsection{Cosmic ray ionization rate}

The $\left[\mathrm{HCO}^{+}\right] /[\mathrm{CO}]$ ratio has been extensively used to estimate the cosmic ray ionization rate. The results of our time dependent model confirms that it is, very likely, the most reliable diagnostic since it reaches the equilibrium in $0.1 \mathrm{Myr}$ and its chemistry is well known. Other $\left[\mathrm{XH}^{+}\right] /[\mathrm{X}]$ ratios, such as $\left[\mathrm{HCS}^{+}\right] /[\mathrm{CS}]$ and $\left[\mathrm{HCNH}^{+}\right] /[\mathrm{HCN}]$, are also expected to be in equilibrium at the typical ages of a molecular cloud. Figure B.4 shows the variation of the $\left[\mathrm{HCO}^{+}\right] /[\mathrm{CO}],\left[\mathrm{HCNH}^{+}\right] /[\mathrm{HCN}]$, and $\left[\mathrm{HCS}^{+}\right] /[\mathrm{CS}]$ as a function of the cosmic ray ionization rate in steady-state calculations. Assuming an uncertainty of a factor of 2 in the derived abundance ratios, values of $\zeta_{\mathrm{H}_{2}}$ in the interval between $3 \times 10^{-17} \mathrm{~s}^{-1}$ to $1 \times 10^{-16} \mathrm{~s}^{-1}$ are consistent with the observed $\left[\mathrm{HCO}^{+}\right] /[\mathrm{CO}]$ and $\left[\mathrm{HCNH}^{+}\right] /[\mathrm{HCN}]$ ratios. These values are at the upper end of the values inferred for cloud cores (Caselli et al. 1998) and are higher than those of the typical dark cloud L1544, $\zeta_{\mathrm{H}_{2}} \sim 1 \times 10^{-18}$ (Galli \& Padovani 2015). This could explain the higher gas and dust temperature in this dense core of $T_{\mathrm{d}} \sim 12 \mathrm{~K}$, compared with dust temperature in L1544 of $T_{\mathrm{d}}<6-8 \mathrm{~K}$ (Crapsi et al. 2007).

The $\left[\mathrm{HCS}^{+}\right] /[\mathrm{CS}]$ ratio is not fitted by any reasonable value of $\zeta_{\mathrm{H}_{2}}$. This is not surprising since the $\mathrm{HCS}^{+}$emission is mainly coming from the low density envelope. In Fig. B.2 we show model predictions for a density of $n\left(\mathrm{H}_{2}\right)=10^{4} \mathrm{~cm}^{-3}$. The abundances of most molecules decrease while the $\mathrm{HCS}^{+}$abundance remains constant and that of CCS increases. These calculations were carried out assuming the same elemental abundances as in the high density case. This is not realistic since lower depletions are expected in the envelope and, therefore, the $\mathrm{HCS}^{+}$ abundance would be higher. Moreover, this core is illuminated on its eastern side by the nearby Herbig Ae stars $\mathrm{LkH} \alpha 327$ and $\mathrm{LkH} \alpha 328$ (Walawender et al. 2009; Young et al. 2015). The 
UV photons are expected to increase the $\mathrm{HCS}^{+}$abundance. In fact, the $\mathrm{HCS}^{+} 2 \rightarrow 1$ emission is maximum toward the illuminated side (see Fig. 2).

Our estimate of the cosmic ray ionization rate permits us to obtain an estimate of the $\mathrm{N}_{2}$ abundance in this core. Since it is one of the main nitrogen reservoirs, $\mathrm{N}_{2}$ cannot be observed at (sub)millimeter wavelengths because of its lack of dipole moment. We can only estimate its abundance indirectly through the protonated compound, $\mathrm{N}_{2} \mathrm{H}^{+}$. The $\mathrm{N}_{2} \mathrm{H}^{+}$abundance is very sensitive to the cosmic ray ionization rate. In Fig. B.4, we present the $\left[\mathrm{N}_{2} \mathrm{H}^{+}\right] /\left[\mathrm{N}_{2}\right]$ ratio as a function of the cosmic ray ionization rate. Assuming $\zeta_{\mathrm{H}_{2}}=4 \times 10^{-17} \mathrm{~s}^{-1}$ and on the basis of our observational data, we derive $\left[\mathrm{N}_{2}\right] \sim 8 \times 10^{-5}$, which is consistent with the typical value of the $\mathrm{N}$ elemental abundance in a dark cloud, $\mathrm{N} / \mathrm{H}=6.4 \times 10^{-5}$.

The $\mathrm{HOCO}^{+}$molecule (protonated carbon dioxide) is thought to form via a standard ion-molecule reaction, the transfer of a proton from $\mathrm{H}_{3}^{+}$to $\mathrm{CO}_{2}$ (see, e.g., Turner et al. 1999). To date $\mathrm{HOCO}^{+}$has been detected toward the Galactic center, a few starless translucent clouds (Turner et al. 1999), a single low-mass Class 0 protostar (Sakai et al. 2008), and the protostellar shock L1157-B1 (Podio et al. 2014). Interestingly, this molecule is particularly abundant, $\sim 10^{-9}$, in the Galactic center region (Minh et al. 1988, 1991; Deguchi et al. 2006; Neill et al. 2014). We derive an $\mathrm{HOCO}^{+}$abundance of $\sim 10^{-11}$, i.e, two orders of magnitude lower than that derived in the Galactic center clouds (Deguchi et al. 2006; Neill et al. 2014) and L1157-B1 (Podio et al. 2014), and a factor of $\sim 10$ lower than that in translucent clouds (Turner et al. 1999). This is not unexpected since the $\mathrm{HOCO}^{+}$abundance is inversely proportional to the gas density (Sakai et al. 2008) and B1b is a dense core. Our model underpredicts the $\mathrm{HOCO}^{+}$abundance by a factor 200 (see Fig. B.2). As commented below, $\mathrm{HOCO}^{+}$is proxy of $\mathrm{CO}_{2}$, which is not efficiently formed in the gas phase (Turner et al. 1999).

The lack of an electric dipole moment of $\mathrm{CO}_{2}$ makes gasphase detections difficult. The abundance of $\mathrm{CO}_{2}$ in the solid phase has been investigated through its two vibrational modes at 4.3 and $15.0 \mu \mathrm{m}$. In the solid phase, the typical abundance is $\sim 10^{-6}$ relative to total $\mathrm{H}_{2}$ (Gerakines et al. 1999; Gibb et al. 2004). In the gas phase, the typical detected abundance is 1-3 $\times 10^{-7}$ (van Dishoeck et al. 1996; Boonman et al. 2003; Sonnentrucker et al. 2006). Assuming the $\left[\mathrm{HOCO}^{+}\right] /\left[\mathrm{CO}_{2}\right]$ ratio predicted by our model, $2.2 \times 10^{-4}$, we estimate a $\mathrm{CO}_{2}$ abundance of $\sim 5 \times 10^{-8}$. This value is at the lower end of those measured in star-forming regions but 200 times larger than that predicted by our gas-phase model. Photodesorption and sputtering of the icy mantles are more likely to be the main $\mathrm{CO}_{2}$ production mechanisms.

\subsection{Sulfur chemistry: $C, O$, and $S$ elemental abundances}

The initial elemental abundances in the gas phase are an essential parameter in the chemical modeling. In cloud cores $\left(A_{v}>\right.$ $10 \mathrm{mag}$ ), as long as the $\mathrm{C}$ elemental abundance is lower than $\mathrm{O}$, we can assume that the abundance of $\mathrm{C}$ is equal to that of $\mathrm{CO}$ and conclude that $\mathrm{C} / \mathrm{H}=1.7 \times 10^{-5}$; we note that fractional abundances are given with respect to $\mathrm{H}_{2}$. The main source of uncertainty in this value comes from the uncertainty in the $\mathrm{CO}$ abundance, which is estimated to be a factor of 2 .

To estimate the amount of $\mathrm{S}$ in the gas phase requires a detailed modeling of its chemistry. As shown in Fig. B.5, the cosmic ray ionization rate is a critical paramenter to estimate the $\mathrm{S} / \mathrm{H}$. The main sulfur reservoirs in a dense core are atomic sulfur (S) and SO (see Fig. 7). Unfortunately, chemical models make a

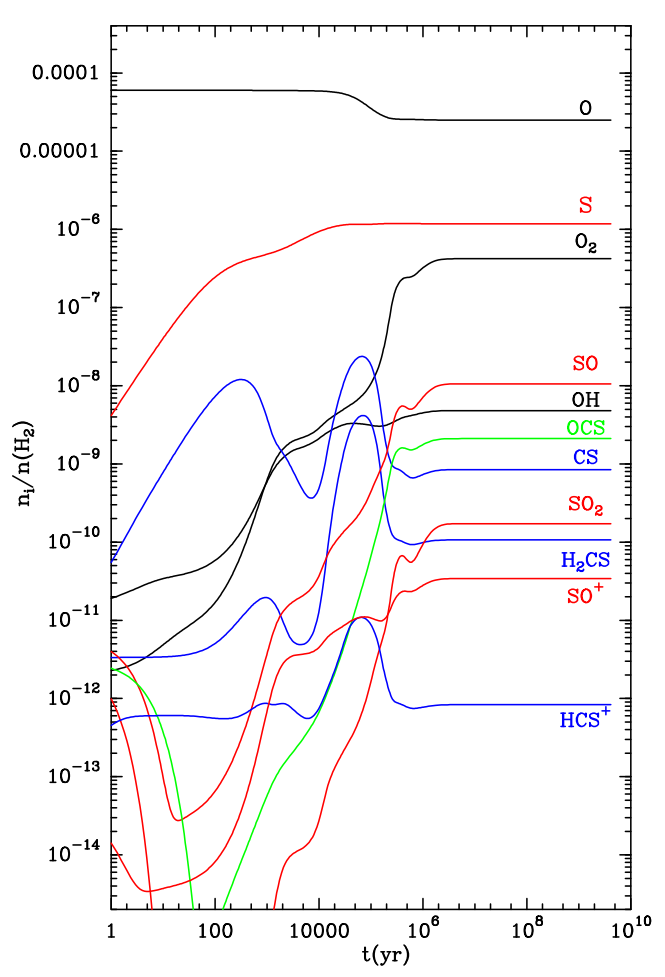

Fig. 7. Predicted fractional abundances using the same parameters as in Fig. B.2. The abundances of $\mathrm{SO}, \mathrm{SO}_{2}$ and $\mathrm{SO}^{+}$, and $\mathrm{O}_{2}$ dramatically increase after 1 Myr.

poor work in predicting the fractional abundance of SO, because the predicted $\mathrm{SO}$ abundance depends on two uncertain factors: i) the $\mathrm{S}+\mathrm{O}_{2} \rightarrow \mathrm{SO}+\mathrm{O}$ and $\mathrm{S}+\mathrm{OH} \rightarrow \mathrm{SO}+\mathrm{H}$ reaction rates and ii) the $\mathrm{O}_{2}$ and $\mathrm{OH}$ abundances.

The $\mathrm{S}+\mathrm{O}_{2}$ reaction rate coefficient at low temperatures is not known. Experimental measurements have only been performed above $200 \mathrm{~K}$. Lu et al. (2004) reviewed the various theoretical and experimental results and adopted a value of $2 \times 10^{-12} \mathrm{~cm}^{3} \mathrm{~s}^{-1}$ at $298 \mathrm{~K}$. The UMIST and KIDA databases suggest different values. In KIDA, a constant value of $2.1 \times$ $10^{-12} \mathrm{~cm}^{3} \mathrm{~s}^{-1}$ is suggested in the $250-430 \mathrm{~K}$ temperature range whereas the latest version of the UMIST database gives $1.76 \times$ $10^{-12}(T / 300)^{0.81} \exp (30.8 / T) \mathrm{cm}^{3} \mathrm{~s}^{-1}$ in the $200-3460 \mathrm{~K}$ temperature interval. This small activation barrier would imply rates as low as $10^{-23} \mathrm{~cm}^{3} \mathrm{~s}^{-1}$ at the temperature of $12 \mathrm{~K}$ and a decrease in the $\mathrm{SO}, \mathrm{SO}_{2}$, and $\mathrm{SO}^{+}$abundances. Quasiclassical trajectory calculations were carried out for the reaction $\mathrm{S}+\mathrm{O}_{2}$ and its reverse by Rodrigues \& Varandas (2003). With 6000 trajectories they obtain that the reaction rate remains constant or decreases very slowly for temperatures between $50 \mathrm{~K}$ and $1000 \mathrm{~K}$. Using the same potential, we performed new quasiclassical trajectory calculations with 100000 trajectories for temperatures between $10 \mathrm{~K}$ and $50 \mathrm{~K}$ (see Appendix A). Our results confirm that the reaction rate remains very constant (within 30\%) in the $10 \mathrm{~K}$ to $50 \mathrm{~K}$ range. Therefore, we adopted the KIDA value, $2.1 \times 10^{-12} \mathrm{~cm}^{3} \mathrm{~s}^{-1}$, in our calculations.

The second more important reaction for the formation of $\mathrm{SO}$ is $\mathrm{S}+\mathrm{OH}$. DeMore et al. (1997) measured a value of $6.6 \times 10^{-11} \mathrm{~cm}^{3} \mathrm{~s}^{-1}$ at $398 \mathrm{~K}$, which is the value adopted in our calculations. Recent theoretical calculations by Goswami et al. (2014) predicted a value a factor of $\sim 2$ lower. These calculations were performed for a range of temperatures between 10-500 K and the reaction rate remains very constant over this range with an increase of a factor of $\sim 1.6$ for temperatures close to $10 \mathrm{~K}$. 
A. Fuente et al.: Ionization fraction and the enhanced sulfur chemistry in Barnard 1

Our adopted experimental value is very likely good by a factor of 2 for the physical conditions of B1.

The oxygen and sulfur chemistries are tightly linked. Since $\mathrm{S}$ is rapidly converted to $\mathrm{SO}$ by reacting with $\mathrm{O}_{2}$ and $\mathrm{OH}$, its abundance is very dependent on the amount of molecular oxygen in the gas phase, that itself depends on the $\mathrm{C} / \mathrm{O}$ ratio (see Fig. B.6). Conversely, a large abundance of $\mathrm{S}$ in the gas phase $\left(>10^{-6}\right)$ leads to a rapid destruction of $\mathrm{O}_{2}$ and decreases the $\left[\mathrm{O}_{2}\right] /[\mathrm{O}]$ ratio (see Fig. B.5). We find a good agreement with the observations, assuming $\mathrm{C} / \mathrm{O}=0.6$ and $\mathrm{S} / \mathrm{H}$ in the interval between $6 \times 10^{-7}$ and $10^{-6}$. With these parameters we have an excellent fit of the $\mathrm{SO}$ abundance and the $[\mathrm{SO}] /[\mathrm{CS}],\left[\mathrm{SO}_{2} /[\mathrm{SO}]\right.$, and $\left[\mathrm{H}_{2} \mathrm{CS}\right] /[\mathrm{CS}]$ ratios, although the agreement is worse for OCS (Figs. B.5 and B.6). We do not have a good explanation for the low observed abundance of OCS. It may be frozen onto the grain mantles in this cold core. Taking into account the limitations of our model, of one single $n\left(\mathrm{H}_{2}\right)$ and $T$, we consider, however, that the overall agreement with observational data is quite good. Assuming these values, our chemical model predicts a steady-state $\mathrm{O}_{2}$ abundance of $\sim 4 \times 10^{-7}$. There is no measurement of the $\mathrm{O}_{2}$ abundance in $\mathrm{B} 1 \mathrm{~b}$, but this predicted abundance is higher than the upper limits derived for other dark clouds (Pagani et al. 2003) and that reported in $\rho$ Oph $\mathrm{A}\left(\sim 5 \times 10^{-8}\right)$ (Liseau et al. 2012). The low $\mathrm{O}_{2}$ abundance measured in dark clouds is usually explained in terms of time evolution. $\mathrm{O}_{2}$ is a late-type molecule that presents low abundance $\left(\sim 10^{-8}\right)$ at typical ages of dark clouds (a few $0.1 \mathrm{Myr}$ ). Another possibility is that $\mathrm{O}_{2}$ becomes frozen on grain mantles. Regarding the sulfur chemistry, a lower $\mathrm{O}_{2}$ abundance would decrease the SO production via the $\mathrm{S}+\mathrm{O}_{2}$ reaction. In this case, we would need to increase $\mathrm{S} / \mathrm{H}$ to reproduce the observations and our estimate would be a lower limit.

Finally, our chemical model does not include surface chemistry. The main effect of dust grains is to decrease the abundance of sulfur bearing molecules, especially $\mathrm{SO}_{2}$, because of adsorption on grain mantles. The most abundant species in Barnard $1 \mathrm{~b}$, $\mathrm{SO}$, is thought to form in the gas phase. As long as this remains true, we consider that our measurement of the sulfur depletion is reliable.

\section{Barnard 1b chemistry}

We have used a state-of-the-art, gas-phase model to derive the cosmic ionization rate and elemental abundances toward the dense core Barnard 1b. Our model fit predicts the observed abundances and abundance ratios reasonably well (within a factor of two), but it fails, by several orders of magnitude to predict the abundances of $\mathrm{HCS}^{+}$and $\mathrm{HOCO}^{+}$. As commented above, $\mathrm{HCS}^{+}$ is very likely coming from the lower density envelope instead of the dense core and cannot be accounted by our single point $(n, T)$ model. In the case of $\mathrm{HOCO}^{+}$, the model failure is not surprising since $\mathrm{CO}_{2}$ is mainly formed by surface reactions.

In Table 4 we compare the initial abundances derived toward B1b with typical values in dark clouds and diffuse clouds (Agúndez \& Wakelam 2013). The depletion of C and O is ten times larger than those measured in prototypical dark clouds such as TMC 1 and L134N. These values are similar to those found in prestellar cores and young stellar objects where high values of $\mathrm{CO}$ depletion $(\sim 5-10)$ and high deuterium fractions ( 0.01) are measured (Caselli et al. 1999; Jørgensen et al. 2002; Crapsi et al. 2005; Emprechtinger et al. 2009; Alonso-Albi et al. 2010). High values of the deuterium fractions are observed in Barnard 1b (Lis et al. 2002; Marcelino et al. 2005), which further confirm this similarity. However, the relatively low
Table 4. Elemental abundances relative to $\mathrm{H}$.

\begin{tabular}{ccc|cc|cc}
\hline \hline \multicolumn{2}{c|}{ Solar } & \multicolumn{2}{|c|}{ Dark cloud: TMC1 } & \multicolumn{2}{|c}{ Barnard 1b } \\
& $\mathrm{X} / \mathrm{H}^{c}$ & $f_{\mathrm{D}}{ }^{d}$ & $\mathrm{X} / \mathrm{H}$ & \multicolumn{1}{c|}{$f_{\mathrm{D}}$} & $\mathrm{X} / \mathrm{H}$ & $f_{\mathrm{D}}$ \\
\hline $\mathrm{C}$ & $1.4 \times 10^{-4}$ & $\sim 1$ & $1.4 \times 10^{-4}$ & $\sim 1$ & $1.7 \times 10^{-5}$ & $\sim 8$ \\
$\mathrm{O}$ & $3.1 \times 10^{-4}$ & $\sim 1$ & $1.3 \times 10^{-4}$ & $\sim 2$ & $3.0 \times 10^{-5}$ & $\sim 10$ \\
$\mathrm{~N}$ & $7.7 \times 10^{-5}$ & $\sim 1$ & $6.2 \times 10^{-5}$ & $\sim 1$ & $6.4 \times 10^{-5}$ & $\sim 1$ \\
$\mathrm{~S}$ & $1.5 \times 10^{-5}$ & $\sim 1$ & $8.0 \times 10^{-8}$ & $\sim 200$ & $6.0 \times 10^{-7}$ & $\sim 25$ \\
\hline
\end{tabular}

Notes. ${ }^{(a)}$ From the compilation by Agúndez \& Wakelam $(2013) ;{ }^{(b)}$ this work; ${ }^{(c)}$ elemental abundance in the gas phase; ${ }^{(d)}$ depletion factor $=$ $(\mathrm{X} / \mathrm{H})_{\odot} /(\mathrm{X} / \mathrm{H})$.

$\mathrm{S}$ depletion in this source is unexpected. A sulfur depletion factor of $\sim 100$ is adopted to explain the chemistry in dark clouds and dense cores (Tafalla et al. 2006; Agúndez \& Wakelam 2013). A higher gas-phase sulfur abundance approaching the solar value of $1.5 \times 10^{-5}$ has only been found in bipolar outflows (Bachiller \& Pérez Gutiérrez 1997; Anderson et al. 2013), photodissociation regions (Goicoechea et al. 2006), and hot cores (Esplugues et al. 2013, 2014). In these cases, this abundance was intrepreted as the consequence of the release of the S-species from the icy grain mantles because of thermal and nonthermal desorption and sputtering. Contrary to dark clouds and prestellar cores, in B1b the S depletion is comparable to that of $\mathrm{C}$ and $\mathrm{O}$, which explains the high abundances of S-bearing species in this core.

The abundance of sulfur in grains is very uncertain. Thus far, the only S-bearing molecule unambiguously detected in ice mantles is OCS, because of its large band strength in the infrared (Geballe et al. 1985; Palumbo et al. 1995), and maybe $\mathrm{SO}_{2}$ (Boogert et al. 1997), but there are only upper limits of the solid $\mathrm{H}_{2} \mathrm{~S}$ abundance (Jiménez-Escobar \& Muñoz Caro 2011), which is thought to be the main sulfur reservoir in the ice. Measurements of the sulfur depletion are derived by comparing the predictions of gas-phase chemical models with the observed abundances of the main S-bearing species. As reported above, chemical models are limited by the uncertainty in the reaction rates and the poorly known $[\mathrm{O}] /\left[\mathrm{O}_{2}\right]$ ratio and the exact value of the sulfur depletion is uncertain. Our results suggest, however, that the sulfur depletion in Barnard $1 \mathrm{~b}$ is not as extreme as in dark clouds where the abundance of S-bearing species is lower.

This moderate sulfur depletion could be the consequence of the star formation activity in the region. B1b is irradiated on its eastern side by UV photons from the nearby Herbig Ae stars $\mathrm{LkH} \alpha 327$ and $\mathrm{LkH} \alpha 328$ (Walawender et al. 2009; Young et al. 2015). On its western side, B1b is impacted by the outflows associated with B1a and B1d (Hatchell et al. 2007). Not unrelated, the level of turbulence in B1 $\left(\sim 0.4 \mathrm{~km} \mathrm{~s}^{-1}\right)$ is higher than in cold cores such as L1544, TMC-1 or L134N $\left(\sim 0.1 \mathrm{~km} \mathrm{~s}^{-1}\right)$. Sputtering induced by collisions may be efficient in this turbulent environment to erode the icy mantles and release the sulfuretted species to the gas phase. Sputtering could also explain the high abundances of complex molecules observed in this core (Marcelino et al. 2009; Cernicharo et al. 2012). The main drawback of this interpretation is that it would also imply a low depletion of $\mathrm{CO}$ that is not observed.

Another possibility is that the moderate sulfur depletion is a consequence of the rapid collapse of B1b. Aikawa et al. (2005) investigated the impact of different collapse timescales on the gas chemistry. The initial conditions of their first model lie close to the critical Bonnor-Ebert sphere with a central density 
of $\sim 10^{4} \mathrm{~cm}^{-3}$. When the central density of the core reaches $10^{5} \mathrm{~cm}^{-3}$, the S-bearing species were totally depleted at $R \sim$ $5000 \mathrm{AU}$. In the second case, internal gravity overwhelms pressure and the collapse is much faster (about $0.1 \mathrm{Myr}$ ). When the central density of the core reaches $10^{5} \mathrm{~cm}^{-3}$, molecules such as $\mathrm{CS}$, SO, and $\mathrm{C}_{2} \mathrm{~S}$ have abundances of $\sim 10^{-10}$ in the core center while $\mathrm{CO}$ is significantly depleted. These abundances are too low compared with our measurements, but this is partially due to the adopted $\mathrm{S}$ elemental abundance in the Aikawa model $(\mathrm{S} / \mathrm{H}=$ $9.14 \times 10^{-8}$ ). We have not targeted the position of the protostar but a nearby position at $R \sim 2500 \mathrm{AU}$, where the sulfur depletions is expected to be lower. Moreover, our position is spatially coincident with the red wing of the B1b-S outflow. Higher spatial resolution observations are needed to determine the possible influence of the B1b-S outflow on the S chemistry.

Summarizing, we propose that the low sulfur depletion in this region could be the result of two different factors, which are both related to the star formation activity. The enhanced UV fields and the surrounding outflows could be the cause of peculiar initial conditions with low sulfur depletion and high abundances of complex molecules. The star formation activity could also have induced a rapid collapse of the B1-b core that preserves the high abundances of the sulfuretted species. The compact outflow associated with B1b-S could also heat the surroundings and contribute to halting the depletion of S-molecules.

\section{Summary and conclusions}

On the basis of our spectral survey toward Barnard 1b, we selected a set of neutral and ionic species to determine the value of the cosmic ray ionization rate and depletion factors of the $\mathrm{C}, \mathrm{N}$, $\mathrm{O}$, and $\mathrm{S}$. These are the parameters that determine the gas ionization fraction and, hence, the dynamical evolution of this core. In our survey, we detected the following ions: $\mathrm{HCO}^{+}, \mathrm{H}^{13} \mathrm{CO}^{+}$, $\mathrm{HC}^{18} \mathrm{O}^{+}, \mathrm{HC}^{17} \mathrm{O}^{+}, \mathrm{DCO}^{+}, \mathrm{D}^{13} \mathrm{CO}^{+}, \mathrm{HOCO}^{+}, \mathrm{DOCO}^{+}, \mathrm{HCS}^{+}$, $\mathrm{SO}^{+}, \mathrm{HC}^{34} \mathrm{~S}^{+}, \mathrm{DCS}^{+}, \mathrm{HCNH}^{+}, \mathrm{H}_{2} \mathrm{COH}^{+}, \mathrm{HC}_{3} \mathrm{NH}^{+}, \mathrm{NNH}^{+}$, $\mathrm{NND}^{+}, \mathrm{N}^{15} \mathrm{NH}^{+}$, and ${ }^{15} \mathrm{NNH}^{+}$. This is the most complete inventory of molecular ions in this core and, probably, the first secure detection of the deuterated ion $\mathrm{DOCO}^{+}$and $\mathrm{DCS}^{+}$in any source.

We use a state-of-the-art, pseudo-time-dependent, gas-phase chemical model that includes the ortho and para forms of $\mathrm{H}_{2}$, $\mathrm{H}_{2}^{+}, \mathrm{D}_{2}^{+}, \mathrm{H}_{3}^{+}, \mathrm{H}_{2} \mathrm{D}^{+}, \mathrm{D}_{2} \mathrm{H}^{+}, \mathrm{D}_{2}$, and $\mathrm{D}_{3}^{+}$. Our model assumes $n\left(\mathrm{H}_{2}\right)=10^{5} \mathrm{~cm}^{-3}$ and $T_{k}=12 \mathrm{~K}$, which is derived from previous works. The observational data are well fitted with $\zeta_{\mathrm{H}_{2}}=$ $(3-10) \times 10^{-17} \mathrm{~s}^{-1}$ and the elemental abundances $\mathrm{O} / \mathrm{H}=3 \times 10^{-5}$, $\mathrm{N} / \mathrm{H}=(6.4-8) \times 10^{-5}, \mathrm{C} / \mathrm{H}=1.7 \times 10^{-5}$, and $\mathrm{S} / \mathrm{H}=(6.0-10) \times$ $10^{-7}$. On basis of the $\mathrm{HOCO}^{+}$and $\mathrm{N}_{2} \mathrm{H}^{+}$observations, we derive abundances of $\sim 5 \times 10^{-8}$ and $(6.4-8) \times 10^{-5}$ for $\mathrm{CO}_{2}$ and $\mathrm{N}_{2}$, respectively.

Barnard $1 \mathrm{~b}$ presents similar depletion of $\mathrm{C}$ and $\mathrm{O}$ as those measured in prestellar cores. The depletion of $\mathrm{S}$ is, however, moderate more similar to that found in bipolar outflows, hot cores, and photon-dominated regions. This high $\mathrm{C}$ and $\mathrm{O}$ depletion, together with the moderate $\mathrm{S}$ depletion, produces a peculiar chemistry that is very rich in sulfuretted species. We propose that the low $\mathrm{S}$ depletion is the consequence of the peculiar initial conditions (important outflows and enhanced UV fields in the surroundings) and a rapid collapse $(\sim 0.1 \mathrm{Myr})$ that allows most $\mathrm{S}$ - and N-bearing species to remain in the gas phase. The compact outflow associated with B1b-S could also contribute to enhance the abundance of S-molecules.

Acknowledgements. We thank the Spanish MINECO for funding support from grants CSD2009-00038, FIS2012-32096, FIS2014-52172-C2 AYA2012-32032, and ERC under ERC-2013-SyG, G. A. 610256 NANOCOSMOS. We thank Prof. J.A.C. Varandas for sending us the Fortran code of their potential energy surface for the $\mathrm{S}+\mathrm{O}_{2}$ reaction. E.R. and M.G. thank the INSU/CNRS program PCMI for funding. This research used the facilities of the Canadian Astronomy Data Centre operated by the National Research Council of Canada with the support of the Canadian Space Agency.

\section{References}

Agúndez, M., \& Wakelam, V. 2013, Chem. Rev., 113, 8710

Agúndez, M., Cernicharo, J., de Vicente, P., et al. 2015, A\&A, 579, L10

Aikawa, Y., Herbst, E., Roberts, H., \& Caselli, P. 2005, ApJ, 620, 330

Alonso-Albi, T., Fuente, A., Crimier, N., et al. 2010, A\&A, 518, A52

Anderson, D. E., Bergin, E. A., Maret, S., \& Wakelam, V. 2013, ApJ, 779, 141

Bachiller, R., \& Cernicharo, J. 1984, A\&A, 140, 414

Bachiller, R., \& Pérez Gutiérrez, M. 1997, ApJ, 487, L93

Bachiller, R., Menten, K. M., \& del Rio Alvarez, S. 1990, A\&A, 236, 461

Bergin, E. A., Plume, R., Williams, J. P., \& Myers, P. C. 1999, ApJ, 512, 724

Boogert, A. C. A., Schutte, W. A., Helmich, F. P., Tielens, A. G. G. M., \& Wooden, D. H. 1997, A\&A, 317, 929

Boonman, A. M. S., Doty, S. D., van Dishoeck, E. F., et al. 2003, A\&A, 406, 937 Caselli, P., Walmsley, C. M., Terzieva, R., \& Herbst, E. 1998, ApJ, 499, 234

Caselli, P., Walmsley, C. M., Tafalla, M., Dore, L., \& Myers, P. C. 1999, ApJ, 523, L165

Caselli, P., Benson, P. J., Myers, P. C., \& Tafalla, M. 2002, ApJ, 572, 238

Cernicharo, J. 2012, in EAS Publ. Ser. 58, eds. C. Stehlé, C. Joblin, \& L. d'Hendecourt, 251

Cernicharo, J., Marcelino, N., Roueff, E., et al. 2012, ApJ, 759, L43

Cernicharo, J., Bailleux, S., Alekseev, E., et al. 2014, ApJ, 795, 40

Cernicharo, J., Tercero, B., Fuente, A., et al. 2013, ApJ, 771, L10

Chin, Y.-N., Henkel, C., Whiteoak, J. B., Langer, N., \& Churchwell, E. B. 1996, A\&A, 305, 960

Crapsi, A., Caselli, P., Walmsley, C. M., et al. 2005, ApJ, 619, 379

Crapsi, A., Caselli, P., Walmsley, M. C., \& Tafalla, M. 2007, A\&A, 470, 221

Dalgarno, A., \& Lepp, S. 1984, ApJ, 287, L47

Daniel, F., Gérin, M., Roueff, E., et al. 2013, A\&A, 560, A3

Deguchi, S., Miyazaki, A., \& Minh, Y. C. 2006, PASJ, 58, 979

DeMore, W. B., Sander, S. P., Golden, D. M., et al. 1997, JPL Publ. 97-4, 1

Dislaire, V., Hily-Blant, P., Faure, A., et al. 2012, A\&A, 537, A20

Dorta-Urra, A., Zanchet A., Roncero, O., \& Aguado, A. 2015, J. Chem. Phys., 142,154301

Emprechtinger, M., Caselli, P., Volgenau, N. H., Stutzki, J., \& Wiedner, M. C. 2009, A\&A, 493, 89

Esplugues, G. B., Tercero, B., Cernicharo, J., et al. 2013, A\&A, 556, A143

Esplugues, G. B., Viti, S., Goicoechea, J. R., \& Cernicharo, J. 2014, A\&A, 567, A95

Fechtenbaum, S., \& Bontemps, S. 2013, in SF2A-2013: Proc. Annual meeting of the French Society of Astronomy and Astrophysics, eds. L. Cambresy, F. Martins, E. Nuss, \& A. Palacios, 219

Flower, D. R., Pineau des Forêts, G., \& Walmsley, C. M. 2004, A\&A, 427, 887 Flower, D. R., Pineau Des Forêts, G., \& Walmsley, C. M. 2006, A\&A, 449, 621 Fuente, A., Rodriguez-Franco, A., Garcia-Burillo, S., Martın-Pintado, J., \& Black, J. H. 2003, A\&A, 406, 899

Galli, D., \& Padovani, M. 2015, ArXiv e-prints [arXiv: 1502.03380]

Geballe, T. R., Baas, F., Greenberg, J. M., \& Schutte, W. 1985, A\&A, 146, L6 Gerakines, P. A., Whittet, D. C. B., Ehrenfreund, P., et al. 1999, ApJ, 522, 357 Gerin, M., Pety, J., Fuente, A., et al. 2015, A\&A, 577, L2

Gibb, E. L., Whittet, D. C. B., Boogert, A. C. A., \& Tielens, A. G. G. M. 2004 , ApJS, 151, 35

Glassgold, A. E., \& Langer, W. D. 1973, ApJ, 179, L147

Glassgold, A. E., Galli, D., \& Padovani, M. 2012, ApJ, 756, 157

Goicoechea, J. R., Pety, J., Gerin, M., et al. 2006, A\&A, 456, 565

Goswami, S., Rajagopala Rao, T., Mahapatra, S., Bussery-Honvault, B., \& Honvault, P. 2014, J. Phys. Chem. A, 118, 5915

Graedel, T. E., Langer, W. D., \& Frerking, M. A. 1982, ApJS, 48, 321

Guelin, M., Langer, W. D., Snell, R. L., \& Wootten, H. A. 1977, ApJ, 217, L165

Hatchell, J., Richer, J. S., Fuller, G. A., et al. 2005, A\&A, 440, 151

Hatchell, J., Fuller, G. A., \& Richer, J. S. 2007, A\&A, 472, 187

Hiramatsu, M., Hirano, N., \& Takakuwa, S. 2010, ApJ, 712, 778

Hirano, N., Kamazaki, T., Mikami, H., Ohashi, N., \& Umemoto, T. 1999, in Star

Formation, ed. T. Nakamoto, 181

Huang, Y.-H., \& Hirano, N. 2013, ApJ, 766, 131

Jiménez-Escobar, A., \& Muñoz Caro, G. M. 2011, A\&A, 536, A91

Jørgensen, J. K., Schöier, F. L., \& van Dishoeck, E. F. 2002, A\&A, 389, 908 Jørgensen, J. K., Harvey, P. M., Evans, II, N. J., et al. 2006, ApJ, 645, 1246 Karplus, M., Porter, R. N., \& Sharma, R. D. 1965, J. Chem. Phys., 43, 3259 Le Bourlot, J. 1991, A\&A, 242, 235

Lis, D. C., Roueff, E., Gerin, M., et al. 2002, ApJ, 571, L55 
A. Fuente et al.: Ionization fraction and the enhanced sulfur chemistry in Barnard 1

Lis, D. C., Wootten, A., Gerin, M., \& Roueff, E. 2010, ApJ, 710, L49

Liseau, R., Goldsmith, P. F., Larsson, B., et al. 2012, A\&A, 541, A73

Lu, C.-W., Wu, Y.-J., Lee, Y.-P., Zhu, R. S., \& Lin, M. C. 2004, J. Chem. Phys. 121,8271

Marcelino, N., Cernicharo, J., Roueff, E., Gerin, M., \& Mauersberger, R. 2005 ApJ, 620, 308

Marcelino, N., Cernicharo, J., Tercero, B., \& Roueff, E. 2009, ApJ, 690, L27

Marcelino, N., Brünken, S., Cernicharo, J., et al. 2010, A\&A, 516, A105

McElroy, D., Walsh, C., Markwick, A. J., et al. 2013, A\&A, 550, A36

McGonagle, D., \& Irvine, W. M. 1997, ApJ, 477, 711

McGonagle, D., Irvine, W. M., \& Ohishi, M. 1994, ApJ, 422, 621

McKee, C. F. 1989, ApJ, 345, 782

Minh, Y. C., Irvine, W. M., \& Ziurys, L. M. 1988, ApJ, 334, 175

Minh, Y. C., Brewer, M. K., Irvine, W. M., Friberg, P., \& Johansson, L. E. B. 1991, A\&A, 244, 470

Neill, J. L., Bergin, E. A., Lis, D. C., et al. 2014, ApJ, 789, 8

Öberg, K. I., Bottinelli, S., Jørgensen, J. K., \& van Dishoeck, E. F. 2010, ApJ, 716,825

Padovani, M., Hennebelle, P., \& Galli, D. 2013, A\&A, 560, A114

Pagani, L., Salez, M., \& Wannier, P. G. 1992, A\&A, 258, 479

Pagani, L., Olofsson, A. O. H., Bergman, P., et al. 2003, A\&A, 402, L77

Palumbo, M. E., Tielens, A. G. G. M., \& Tokunaga, A. T. 1995, ApJ, 449, 674

Pety, J. 2005, in SF2A-2005: Semaine de l'Astrophysique Française, eds.

F. Casoli, T. Contini, J. M. Hameury, \& L. Pagani, 721
Pezzuto, S., Elia, D., Schisano, E., et al. 2012, A\&A, 547, A54

Podio, L., Lefloch, B., Ceccarelli, C., Codella, C., \& Bachiller, R. 2014, A\&A 565, A64

Rizzo, J. R., Fuente, A., Rodríguez-Franco, A., \& García-Burillo, S. 2003, ApJ, 597, L153

Rodrigues, S. P. J., \& Varandas, A. J. C. 2003, J. Phys. Chem. A, 107, 5369

Rodrigues, S. P. J., Sabín, J. A., \& Varandas, A. J. C. 2002, J. Phys. Chem. A, 106,556

Sakai, N., Sakai, T., Aikawa, Y., \& Yamamoto, S. 2008, ApJ, 675, L89

Sonnentrucker, P., González-Alfonso, E., Neufeld, D. A., et al. 2006, ApJ, 650, L71

Tafalla, M., Santiago-García, J., Myers, P. C., et al. 2006, A\&A, 455, 577

Turner, B. E., Terzieva, R., \& Herbst, E. 1999, ApJ, 518, 699

van Dishoeck, E. F., Helmich, F. P., de Graauw, T., et al. 1996, A\&A, 315, L349

Wakelam, V., Herbst, E., Le Bourlot, J., et al. 2010, A\&A, 517, A21

Wakelam, V., Smith, I. W. M., Loison, J.-C., et al. 2013, ArXiv e-prints [arXiv: 1310.4350 ]

Walawender, J., Reipurth, B., \& Bally, J. 2009, AJ, 137, 3254

Wilson, T. L., Mauersberger, R., Gensheimer, P. D., Muders, D., \& Bieging, J. H. 1999, ApJ, 525, 343

Wootten, A., Snell, R., \& Glassgold, A. E. 1979, ApJ, 234, 876

Young, K. E., Young, C. H., Lai, S.-P., Dunham, M. M., \& Evans, II, N. J. 2015, AJ, 150, 40

Zymak, I., Hejduk, M., Mulin, D., et al. 2013, ApJ, 768, 86 


\section{Appendix A}

The $\mathrm{S}+\mathrm{O}_{2} \rightarrow \mathrm{SO}+\mathrm{O}$ reaction was studied using a quasiclassical method with the most up-to-date potential energy surface for the ground singlet state of Rodrigues et al. (2002). This reaction is exothermic by $2345 \mathrm{~K}$ and presents a $\mathrm{SO}_{2}$ deep well with a depth of $10831 \mathrm{~K}$, and there is no barrier along the minimum energy path when $\mathrm{S}$ approaches $\mathrm{O}_{2}$. This potential, however, presents several minima connected among them with saddle points.

A quasiclassical method was employed to calculate the rates for selected states of $\mathrm{O}_{2}(v, j)$ and for temperatures in the 10$300 \mathrm{~K}$ temperature interval. This method is very analogous to that employed by Rodrigues \& Varandas (2003) for higher temperatures. The MIQCT code was used (Dorta-Urra et al. 2015) in which the initial conditions for a particular rovibrational state of $\mathrm{O}_{2}$ are determined according to Karpus and coworkers (Karplus et al. 1965). The $E_{v j}$ energies of $\mathrm{O}_{2}$ are determined by solving numerically the monodimensional quantum Schrödinger equation. The initial translational energy between the reagents is sampled from a Maxwell-Boltzmann distribution. A set of $N_{t}=10^{5}$ initial conditions are propagated for each initial rovibrational state and temperature considered. The state-selected rate coefficients are then obtained as

$k_{v j}(T)=g_{\mathrm{e}}(T) \sqrt{\frac{8 k_{\mathrm{B}} T}{\pi \mu}} \frac{N_{\mathrm{r}}}{N_{\mathrm{t}}} \pi b_{\max }^{2}$,

where $N_{\mathrm{r}}$ denotes the number of reactive trajectories and $b_{\max }$ is the maximum impact parameter for which reaction takes place. Finally, $g_{\mathrm{e}}$ is the electronic partition function obtained assuming that only the ground singlet state, of the 27 spinorbit states of the $\mathrm{S}\left({ }^{3} P\right)+\mathrm{O}_{2}\left({ }^{3} \Sigma_{\mathrm{g}}^{-}\right)$, is reactive, taking the form (Rodrigues \& Varandas 2003)

$g_{\mathrm{e}}(T)=\left\{3\left(5+3 \mathrm{e}^{-570 / T}+\mathrm{e}^{-825 / T}\right)\right\}^{-1}$.

The results obtained are shown in Fig. A.1.

For the initial rotational state, $j=0$, the rate increases with temperature, while for $j=1$ or 2 the rate is nearly constant. This behavior is attributed to the series of wells and saddle points in the entrance channel: when $\mathrm{O}_{2}$ is not rotationally excited and finds one of these barriers is reflected back. On the contrary, when $\mathrm{O}_{2}$ is rotationally excited it overcomes those barriers leading to reaction. This is not a simple question of excess of energy, since the rate also increases with temperature for $v=1$ or 2 and $j=0$.

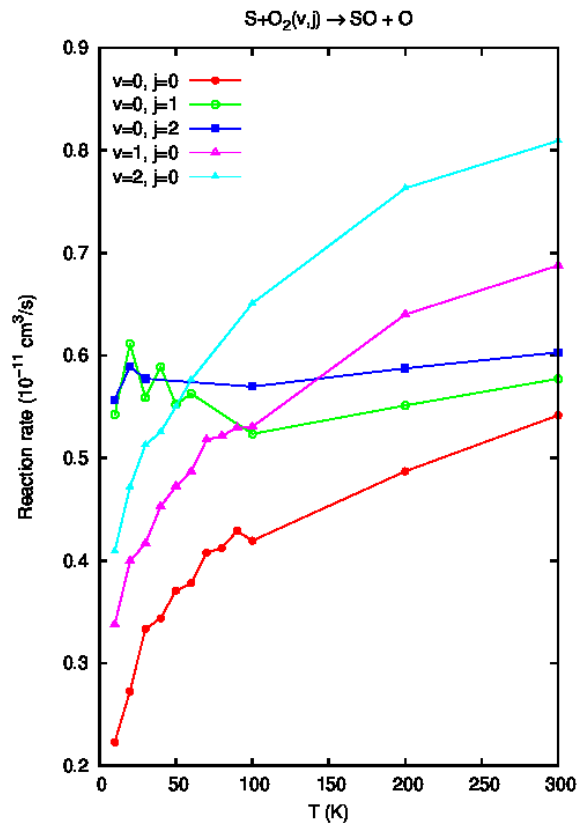

Fig. A.1. State-selected reaction rates for $\mathrm{O}_{2}(v, j)+\mathrm{S} \rightarrow \mathrm{SO}+\mathrm{O}$ collisions as a function of the translational temperatures for selected initial rovibrational states of $\mathrm{O}_{2}$.

At $10 \mathrm{~K}$, the population in $j=0$ is less than $20 \%$, and the average reaction rate is $4.8 \times 10^{-12} \mathrm{~cm}^{3} \mathrm{~s}^{-1}$, which is higher than the value in the KIDA datebase of $2.1 \times 10^{-12} \mathrm{~cm}^{3} \mathrm{~s}^{-1}$, and can be considered approximately constant since it only varies between 4.8 and 5.5 for temperatures between 10 and $50 \mathrm{~K}$. 


\section{Appendix B: Additional tables and figures}

Table B.1. Line integrated intensities.

\begin{tabular}{|c|c|c|c|c|c|c|}
\hline Mol. & Trans. & $\begin{array}{l}\text { Freq. } \\
(\mathrm{MHz})\end{array}$ & $\begin{array}{c}E_{u} \\
(\mathrm{~K}) \\
\end{array}$ & $\begin{array}{c}6.5 \mathrm{~km} \mathrm{~s}^{-1} \\
\text { Area }\left(\mathrm{K} \mathrm{km} \mathrm{s}^{-1}\right)\end{array}$ & $\begin{array}{c}7.0 \mathrm{~km} \mathrm{~s}^{-1} \\
\text { Area }\left(\mathrm{K} \mathrm{km} \mathrm{s}^{-1}\right)\end{array}$ & \\
\hline \multirow[t]{2}{*}{$\mathrm{C}^{18} \mathrm{O}$} & $1 \rightarrow 0$ & 109782.16 & 5.3 & $1.520(0.001)$ & $3.559(0.001)$ & \\
\hline & $2 \rightarrow 1$ & 219460.36 & 15.8 & $0.568(0.008)$ & $3.088(0.010)$ & \\
\hline \multirow[t]{2}{*}{${ }^{13} \mathrm{C}^{18} \mathrm{O}$} & $1 \rightarrow 0$ & 104711.39 & 5.0 & $0.029(0.002)$ & $0.054(0.003)$ & \\
\hline & $2 \rightarrow 1$ & 209419.16 & 15.1 & $0.019(0.006)$ & $0.085(0.007)$ & \\
\hline $\mathrm{C}^{17} \mathrm{O}$ & $1 \rightarrow 0$ & 112359.29 & 5.4 & $0.091(0.004)$ & $0.756(0.005)$ & \\
\hline \multirow[t]{2}{*}{$\mathrm{H}^{13} \mathrm{CO}^{+}$} & $1 \rightarrow 0$ & 86754.29 & 4.2 & $1.545(0.002)$ & $1.553(0.003)$ & \\
\hline & $3 \rightarrow 2$ & 260255.34 & 25.0 & $0.329(0.005)$ & $0.323(0.006)$ & \\
\hline \multirow[t]{2}{*}{$\mathrm{HC}^{18} \mathrm{O}^{+}$} & $1 \rightarrow 0$ & 85162.22 & 4.1 & $0.209(0.002)$ & $0.148(0.003)$ & \\
\hline & $3 \rightarrow 2$ & 255479.39 & 24.5 & $0.042(0.005)$ & $0.010(0.006)$ & \\
\hline $\mathrm{HC}^{17} \mathrm{O}^{+}$ & $1 \rightarrow 0$ & 87057.53 & 4.2 & $0.012(0.003)$ & $0.028(0.003)$ & \\
\hline \multirow[t]{2}{*}{$\mathrm{DCO}^{+}$} & $2 \rightarrow 1$ & 144077.24 & 10.4 & $1.943(0.248)$ & $2.579(0.248)$ & Component at $\sim 6.0 \mathrm{~km} \mathrm{~s}^{-1}$ \\
\hline & $3 \rightarrow 2$ & 216112.58 & 20.7 & $1.535(0.004)$ & $2.008(0.004)$ & \\
\hline \multirow[t]{2}{*}{$\mathrm{D}^{13} \mathrm{CO}^{+}$} & $2 \rightarrow 1$ & 141465.13 & 10.2 & $0.169(0.006)$ & $0.342(0.008)$ & \\
\hline & $3 \rightarrow 2$ & 212194.49 & 20.4 & $0.103(0.003)$ & $0.080(0.006)$ & \\
\hline \multirow[t]{3}{*}{$\mathrm{HOCO}^{+}$} & $4_{0,4} \rightarrow 3_{0,3}$ & 85531.51 & 10.3 & $0.082(0.002)$ & $0.043(0.002)$ & \\
\hline & $5_{0,5}^{0,7} \rightarrow 4_{0,4}$ & 106913.56 & 15.4 & $0.077(0.002)$ & $0.025(0.003)$ & \\
\hline & $10_{0,10} \rightarrow 9_{0,9}$ & 213813.385 & 56.4 & $0.010(0.003)$ & $0.001(0.002)$ & \\
\hline $\mathrm{DOCO}^{+}$ & $5_{0,5} \rightarrow 4_{0,4}$ & 100359.55 & 14.5 & $0.008(0.001)$ & $0.005(0.002)$ & \\
\hline \multirow[t]{13}{*}{$\mathrm{SO}$} & $2_{2} \rightarrow 1_{1}$ & 86093.96 & 19.3 & $1.322(0.001)$ & $0.573(0.001)$ & \\
\hline & $2_{3} \rightarrow 1_{2}$ & 99299.89 & 9.2 & $3.208(0.001)$ & $2.410(0.002)$ & \\
\hline & $5_{4} \rightarrow 4_{4}$ & 100029.55 & 38.6 & $0.048(0.002)$ & $0.004(0.002)$ & \\
\hline & $3_{2} \rightarrow 2_{1}$ & 109252.18 & 21.1 & $1.393(0.003)$ & $0.533(0.004)$ & \\
\hline & $3_{4} \rightarrow 2_{3}$ & $138,178.65$ & 15.9 & $2.021(0.005)$ & $2.129(0.005)$ & \\
\hline & $4_{3} \rightarrow 3_{2}$ & 158971.81 & 28.7 & $0.928(0.002)$ & $1.009(0.003)$ & \\
\hline & $5_{4} \rightarrow 4_{3}$ & 206176.01 & 38.6 & $0.717(0.006)$ & $0.240(0.007)$ & \\
\hline & $5_{5} \rightarrow 4_{4}$ & 215220.65 & 44.1 & $0.613(0.007)$ & $0.205(0.008)$ & \\
\hline & $5_{6} \rightarrow 4_{5}$ & 219949.39 & 35.0 & $1.271(0.007)$ & $0.573(0.009)$ & \\
\hline & $2_{1} \rightarrow 1_{2}$ & 236452.29 & 15.8 & $0.098(0.009)$ & $0.033(0.010)$ & \\
\hline & $3_{2} \rightarrow 2_{3}$ & 246404.59 & 21.1 & $0.040(0.006)$ & $0.035(0.007)$ & \\
\hline & $6_{7} \rightarrow 5_{6}$ & 261843.70 & 47.6 & $0.813(0.008)$ & $0.312(0.010)$ & \\
\hline & $4_{3} \rightarrow 3_{4}$ & 267197.74 & 28.7 & $0.010(0.007)$ & $0.025(0.008)$ & \\
\hline \multirow[t]{12}{*}{${ }^{34} \mathrm{SO}$} & $2_{2} \rightarrow 1_{1}$ & 84410.68 & 19.2 & $0.071(0.002)$ & $0.020(0.002)$ & \\
\hline & $2_{3} \rightarrow 1_{2}$ & 97715.40 & 9.1 & $0.748(0.002)$ & $0.349(0.002)$ & \\
\hline & $3_{2} \rightarrow 2_{1}$ & 106743.36 & 20.9 & $0.090(0.002)$ & $0.018(0.002)$ & \\
\hline & $3_{4} \rightarrow 2_{3}$ & 135775.65 & 15.6 & $0.578(0.003)$ & $0.170(0.004)$ & \\
\hline & $4_{3} \rightarrow 3_{2}$ & 155506.80 & 28.4 & $0.071(0.006)$ & $0.062(0.008)$ & \\
\hline & $4_{4} \rightarrow 3_{3}$ & 168815.11 & 33.4 & $0.069(0.008)$ & $0.018(0.009)$ & \\
\hline & $5_{4} \rightarrow 4_{3}$ & 201846.65 & 38.1 & $0.047(0.008)$ & $0.001(0.009)$ & \\
\hline & $5_{5} \rightarrow 4_{4}$ & 211013.02 & 43.5 & $0.049(0.005)$ & $0.001(0.005)$ & \\
\hline & $5_{6} \rightarrow 4_{5}$ & 215839.92 & 34.4 & $0.142(0.005)$ & $0.060(0.006)$ & \\
\hline & $6_{5} \rightarrow 5_{4}$ & 246663.39 & 49.9 & $0.017(0.005)$ & $0.004(0.006)$ & \\
\hline & $6_{6} \rightarrow 5_{5}$ & 253207.02 & 55.7 & $0.001(0.006)$ & $0.020(0.004)$ & \\
\hline & $6_{7} \rightarrow 5_{6}$ & 256877.81 & 46.7 & $0.044(0.005)$ & $0.016(0.006)$ & \\
\hline \multirow[t]{13}{*}{${ }^{33} \mathrm{SO}$} & $237 / 2 \rightarrow 127 / 2$ & 98443.84 & 9.2 & $0.006(0.001)$ & $0.001(0.002)$ & \\
\hline & $235 / 2 \rightarrow 125 / 2$ & 98455.19 & 9.2 & $0.008(0.001)$ & $0.001(0.002)$ & \\
\hline & $233 / 2 \rightarrow 123 / 2$ & 98460.49 & 9.2 & $0.004(0.002)$ & $0.007(0.002)$ & \\
\hline & $233 / 2 \rightarrow 121 / 2$ & 98474.60 & 9.2 & $0.024(0.001)$ & $0.001(0.002)$ & \\
\hline & $235 / 2 \rightarrow 123 / 2$ & 98482.30 & 9.2 & $0.039(0.002)$ & $0.001(0.002)$ & \\
\hline & $237 / 2 \rightarrow 125 / 2$ & 98489.23 & 9.2 & $0.047(0.001)$ & $0.011(0.004)$ & \\
\hline & $239 / 2 \rightarrow 127 / 2$ & 98493.64 & 9.2 & $0.074(0.001)$ & $0.011(0.004)$ & \\
\hline & $327 / 2 \rightarrow 215 / 2$ & 107953.80 & 21.0 & $0.006(0.002)$ & $0.001(0.007)$ & \\
\hline & $347 / 2 \rightarrow 235 / 2$ & 136939.36 & 15.7 & $0.035(0.004)$ & $0.007(0.005)$ & \\
\hline & $349 / 2 \rightarrow 237 / 2$ & 136943.67 & 15.7 & $0.014(0.014)$ & $0.039(0.005)$ & Component at $\sim 6.0 \mathrm{~km} \mathrm{~s}^{-1}$ \\
\hline & $3411 / 2 \rightarrow 239 / 2$ & 136946.19 & 15.7 & $0.041(0.004)$ & $0.025(0.005)$ & \\
\hline & $437 / 2 \rightarrow 235 / 2$ & 157173.54 & 28.5 & $0.023(0.004)$ & $0.001(0.005)$ & \\
\hline & $435 / 2 \rightarrow 233 / 2$ & 157173.54 & 28.5 & & & \\
\hline $\mathrm{S}^{18} \mathrm{O}$ & $2_{3} \rightarrow 1_{2}$ & 93267.38 & 8.7 & $0.162(0.002)$ & $0.052(0.002)$ & \\
\hline
\end{tabular}


Table B.1. continued.

\begin{tabular}{|c|c|c|c|c|c|c|}
\hline Mol. & Trans. & $\begin{array}{l}\text { Freq. } \\
(\mathrm{MHz})\end{array}$ & $\begin{array}{c}E_{u} \\
(\mathrm{~K})\end{array}$ & $\begin{array}{c}6.5 \mathrm{~km} \mathrm{~s}^{-1} \\
\text { Area }\left(\mathrm{K} \mathrm{km} \mathrm{s}^{-1}\right)\end{array}$ & $\begin{array}{c}7.0 \mathrm{~km} \mathrm{~s}^{-1} \\
\text { Area }\left(\mathrm{K} \mathrm{km} \mathrm{s}^{-1}\right)\end{array}$ & \\
\hline \multirow{13}{*}{$\mathrm{SO}^{+}$} & $3_{2} \rightarrow 2_{1}$ & 99803.66 & 20.5 & $0.012(0.001)$ & $0.001(0.004)$ & \\
\hline & $4_{3} \rightarrow 3_{2}$ & 145874.49 & 27.5 & $0.015(0.004)$ & $0.008(0.005)$ & \\
\hline & $4_{4} \rightarrow 3_{3}$ & 159428.31 & 32.4 & $0.015(0.005)$ & $0.001(0.005)$ & \\
\hline & $4_{5} \rightarrow 34$ & 166285.31 & 22.9 & $0.061(0.005)$ & $0.058(0.007)$ & \\
\hline & $5_{6} \rightarrow 4_{5}$ & 204387.94 & 32.7 & $0.027(0.008)$ & $0.012(0.009)$ & \\
\hline & $5 / 2 \rightarrow 3 / 2(\mathrm{e})$ & 115804.40 & 8.9 & $0.157(0.008)$ & $0.111(0.010)$ & \\
\hline & $5 / 2 \rightarrow 3 / 2(\mathrm{f})$ & 116179.95 & 8.9 & $0.196(0.007)$ & $0.071(0.008)$ & \\
\hline & $7 / 2 \rightarrow 5 / 2(\mathrm{e})$ & 162198.60 & 16.7 & $0.075(0.003)$ & $0.115(0.004)$ & \\
\hline & $7 / 2 \rightarrow 5 / 2(f)$ & 162574.06 & 16.7 & $0.084(0.004)$ & $0.117(0.004)$ & \\
\hline & $9 / 2 \rightarrow 7 / 2(\mathrm{e})$ & 208590.02 & 26.7 & $0.079(0.006)$ & $0.008(0.007)$ & \\
\hline & $9 / 2 \rightarrow 7 / 2(f)$ & 208965.42 & 26.8 & $0.067(0.005)$ & $0.020(0.006)$ & \\
\hline & $11 / 2 \rightarrow 9 / 2(\mathrm{e})$ & 254977.93 & 38.9 & $0.025(0.005)$ & $0.019(0.006)$ & \\
\hline & $11 / 2 \rightarrow 9 / 2(\mathrm{f})$ & 255353.24 & 39.0 & $0.026(0.005)$ & $0.019(0.006)$ & \\
\hline \multirow[t]{3}{*}{$\mathrm{CS}$} & $2 \rightarrow 1$ & 97980.95 & 7.1 & $2.250(0.038)$ & $1.064(0.001)$ & Self-absorbed \\
\hline & $3 \rightarrow 2$ & 146969.02 & 14.1 & $1.301(0.036)$ & $1.483(0.043)$ & Self-absorbed \\
\hline & $5 \rightarrow 4$ & 244935.55 & 35.3 & $0.676(0.021)$ & $0.455(0.025)$ & Bad fit \\
\hline \multirow[t]{3}{*}{$\mathrm{C}^{34} \mathrm{~S}$} & $2 \rightarrow 1$ & 96412.95 & 6.9 & $0.578(0.003)$ & $0.505(0.004)$ & \\
\hline & $3 \rightarrow 2$ & 144617.10 & 13.9 & $0.298(0.006)$ & $0.213(0.006)$ & \\
\hline & $5 \rightarrow 4$ & 241016.09 & 34.7 & $0.038(0.006)$ & $0.021(0.007)$ & \\
\hline \multirow[t]{3}{*}{${ }^{13} \mathrm{CS}$} & $2 \rightarrow 1$ & 92494.27 & 6.7 & $0.259(0.004)$ & $0.159(0.005)$ & \\
\hline & $3 \rightarrow 2$ & 138739.26 & 13.3 & $0.070(0.004)$ & $0.162(0.005)$ & \\
\hline & $5 \rightarrow 4$ & 231220.68 & 33.3 & $0.032(0.006)$ & $0.001(0.027)$ & \\
\hline${ }^{13} C^{34} S$ & $2 \rightarrow 1$ & 90925.99 & 6.5 & $0.009(0.003)$ & $0.007(0.003)$ & \\
\hline \multirow{2}{*}{$\mathrm{C}^{33} \mathrm{~S}^{\mathrm{S}}$} & $2 \rightarrow 1$ & 97172.06 & 7.0 & $0.103(0.006)$ & $0.085(0.007)$ & \\
\hline & $3 \rightarrow 2$ & 145755.73 & 14.0 & $0.044(0.006)$ & $0.037(0.007)$ & Bad fit \\
\hline \multirow[t]{4}{*}{$\mathrm{HCS}^{+}$} & $2 \rightarrow 1$ & 85347.87 & 6.1 & $0.122(0.002)$ & $0.110(0.002)$ & \\
\hline & $4 \rightarrow 3$ & 170691.62 & 20.5 & $0.014(0.025)$ & $0.137(0.029)$ & \\
\hline & $5 \rightarrow 4$ & 213360.65 & 30.7 & $0.037(0.004)$ & $0.048(0.005)$ & \\
\hline & $6 \rightarrow 5$ & 256027.11 & 43.0 & $0.016(0.004)$ & $0.001(0.005)$ & \\
\hline $\mathrm{HC}^{34} \mathrm{~S}^{+}$ & $2 \rightarrow 1$ & 83965.63 & 6.0 & $0.017(0.002)$ & $0.001(0.002)$ & \\
\hline $\mathrm{DCS}^{+}$ & $3 \rightarrow 2$ & 108108.01 & 10.4 & $0.008(0.002)$ & $0.001(0.002)$ & \\
\hline \multirow{2}{*}{$\mathrm{HCNH}^{+}$} & $2 \rightarrow 1$ & 148221.46 & 10.7 & $0.032(0.003)$ & $0.032(0.004)$ & \\
\hline & $3 \rightarrow 2$ & 222329.30 & 21.3 & $0.050(0.007)$ & $0.022(0.008)$ & \\
\hline \multirow{4}{*}{$\mathrm{o}-\mathrm{H}_{2}{ }^{13} \mathrm{CO}$} & $2_{12} \rightarrow 1_{11}$ & 137449.95 & 6.6 & $0.135(0.007)$ & $0.149(0.008)$ & \\
\hline & $2_{11} \rightarrow 1_{10}$ & 146635.67 & 7.3 & $0.118(0.005)$ & $0.064(0.006)$ & Red wing \\
\hline & $3_{13} \rightarrow 2_{12}$ & 206131.62 & 16.5 & $0.074(0.006)$ & $0.041(0.007)$ & \\
\hline & $3_{12} \rightarrow 2_{11}$ & 219908.48 & 17.8 & $0.040(0.005)$ & $0.033(0.005)$ & \\
\hline \multirow[t]{2}{*}{$\mathrm{p}-\mathrm{H}_{2}{ }^{13} \mathrm{CO}$} & $2_{02} \rightarrow 1_{01}$ & 141983.75 & 10.2 & $0.079(0.006)$ & $0.072(0.007)$ & \\
\hline & $3_{03} \rightarrow 2_{02}$ & 212811.19 & 20.4 & $0.053(0.007)$ & $0.016(0.009)$ & \\
\hline \multirow[t]{3}{*}{$\mathrm{H}_{2} \mathrm{COH}^{+}$} & $1_{1,0} \rightarrow 1_{0,1}$ & 168401.14 & 11.1 & $0.012(0.006)$ & $0.019(0.008)$ & \\
\hline & $1_{1,1} \rightarrow 0_{0,0}$ & 226746.31 & 10.9 & $0.001(0.004)$ & $0.021(0.008)$ & \\
\hline & $4_{0,4} \rightarrow 3_{0,3}$ & 252870.34 & 30.4 & $0.001(0.006)$ & $0.018(0.006)$ & \\
\hline \multirow[t]{6}{*}{ OCS } & $7 \rightarrow 6$ & 85139.10 & 16.3 & $0.174(0.002)$ & $0.007(0.003)$ & \\
\hline & $8 \rightarrow 7$ & 97301.21 & 21.0 & $0.167(0.002)$ & $0.058(0.003)$ & \\
\hline & $9 \rightarrow 8$ & 109463.06 & 26.3 & $0.130(0.002)$ & $0.055(0.003)$ & \\
\hline & $11 \rightarrow 10$ & 133785.90 & 38.5 & $0.055(0.004)$ & $0.055(0.005)$ & Red wing \\
\hline & $12 \rightarrow 11$ & 145946.81 & 45.5 & $0.034(0.004)$ & $0.043(0.005)$ & \\
\hline & $13 \rightarrow 12$ & 158107.36 & 53.1 & $0.042(0.005)$ & $0.029(0.006)$ & \\
\hline \multirow[t]{3}{*}{$\mathrm{OC}^{34} \mathrm{~S}$} & $7 \rightarrow 6$ & 83057.97 & 15.9 & $0.011(0.003)$ & & \\
\hline & $8 \rightarrow 7$ & 94922.80 & 20.5 & $0.009(0.002)$ & & \\
\hline & $9 \rightarrow 8$ & 106787.39 & 25.6 & $0.006(0.003)$ & & \\
\hline \multirow[t]{3}{*}{$\mathrm{H}_{2}{ }^{13} \mathrm{CS}$} & $3_{1,3} \rightarrow 2_{1,2}$ & 97632.20 & 7.8 & $0.010(0.002)$ & & \\
\hline & $3_{1,2} \rightarrow 2_{1,1}$ & 100534.75 & 8.0 & $0.007(0.001)$ & & \\
\hline & $3_{0,3} \rightarrow 2_{0,2}$ & 99077.84 & 9.5 & $0.006(0.002)$ & & \\
\hline \multirow[t]{5}{*}{$\mathrm{SO}_{2}$} & $8_{1,7} \rightarrow 8_{0,8}$ & 83688.09 & 36.7 & $0.079(0.003)$ & $0.028(0.004)$ & \\
\hline & $2_{2,0} \rightarrow 3_{1,3}$ & 100878.11 & 12.6 & $0.013(0.002)$ & & \\
\hline & $3_{1,3} \rightarrow 2_{0,2}$ & 104029.43 & 7.7 & $0.677(0.002)$ & $0.216(0.002)$ & \\
\hline & $10_{1,9} \rightarrow 10_{0,10}$ & 104239.30 & 54.7 & $0.020(0.002)$ & & \\
\hline & $8_{0,8} \rightarrow 7_{1,7}$ & 116980.45 & 32.7 & $0.197(0.015)$ & & \\
\hline
\end{tabular}


Table B.1. continued.

\begin{tabular}{|c|c|c|c|c|c|c|}
\hline Mol. & Trans. & $\begin{array}{l}\text { Freq. } \\
(\mathrm{MHz})\end{array}$ & $\begin{array}{c}E_{u} \\
(\mathrm{~K}) \\
\end{array}$ & $\begin{array}{c}6.5 \mathrm{~km} \mathrm{~s}^{-1} \\
\text { Area }\left(\mathrm{K} \mathrm{km} \mathrm{s}^{-1}\right)\end{array}$ & $\begin{array}{c}7.0 \mathrm{~km} \mathrm{~s}^{-1} \\
\text { Area }\left(\mathrm{K} \mathrm{km} \mathrm{s}^{-1}\right)\end{array}$ & \\
\hline & $5_{1,5} \rightarrow 4_{0,4}$ & 135696.02 & 15.7 & $0.453(0.005)$ & $0.133(0.005)$ & \\
\hline & $6_{2,4} \rightarrow 6_{1,5}$ & 140306.17 & 29.2 & $0.077(0.004)$ & $0.026(0.005)$ & Red wing \\
\hline & $4_{2,2} \rightarrow 4_{1,3}$ & 146605.52 & 19.0 & $0.168(0.005)$ & & \\
\hline & $2_{2,0} \rightarrow 2_{1,1}$ & 151378.66 & 12.6 & $0.131(0.005)$ & $0.052(0.006)$ & \\
\hline & $3_{2,2} \rightarrow 3_{1,3}$ & 158199.78 & 15.3 & $0.222(0.005)$ & & \\
\hline & $10_{0,10} \rightarrow 9_{1,9}$ & 160827.84 & 49.7 & $0.060(0.007)$ & & \\
\hline & $5_{2,4} \rightarrow 5_{1,5}$ & 165144.65 & 23.6 & $0.127(0.006)$ & & \\
\hline & $7_{1,7} \rightarrow 6_{0,6}$ & 165225.45 & 27.1 & $0.239(0.006)$ & & \\
\hline & $3_{2,2} \rightarrow 2_{1,1}$ & 208700.34 & 15.3 & $0.237(0.006)$ & $0.095(0.007)$ & \\
\hline & $4_{2,2} \rightarrow 3_{1,3}$ & 235151.72 & 19.0 & $0.242(0.007)$ & $0.042(0.008)$ & \\
\hline & $5_{2,4} \rightarrow 4_{1,3}$ & 241615.80 & 23.6 & $0.154(0.006)$ & $0.042(0.007)$ & \\
\hline & $6_{3,3} \rightarrow 6_{2,4}$ & 254280.54 & 41.4 & $0.019(0.005)$ & & \\
\hline & $4_{3,1} \rightarrow 4_{2,2}$ & 255553.30 & 31.3 & $0.020(0.005)$ & & \\
\hline & $3_{3,1} \rightarrow 3_{2,2}$ & 255958.04 & 27.6 & $0.029(0.005)$ & & \\
\hline & $5_{3,3} \rightarrow 5_{2,4}$ & 256246.95 & 35.9 & $0.021(0.005)$ & & \\
\hline & $7_{2,6} \rightarrow 6_{1,5}$ & 271529.02 & 35.5 & $0.050(0.006)$ & $0.041(0.007)$ & \\
\hline \multirow[t]{4}{*}{${ }^{34} \mathrm{SO}_{2}$} & $3_{1,3} \rightarrow 2_{0,2}$ & 102031.88 & 7.6 & $0.052(0.003)$ & & \\
\hline & $5_{1,5} \rightarrow 4_{0,4}$ & 133471.43 & 15.5 & $0.041(0.005)$ & & \\
\hline & $4_{2,2} \rightarrow 4_{1,3}$ & 141158.94 & 18.7 & $0.010(0.004)$ & & \\
\hline & $3_{2,2} \rightarrow 2_{1,1}$ & 203225.06 & 15.0 & $0.035(0.010)$ & & \\
\hline \multirow[t]{13}{*}{ CCS } & $7_{6} \rightarrow 6_{5}$ & 86181.41 & 23.3 & $0.162(0.003)$ & $0.111(0.003)$ & \\
\hline & $7_{7} \rightarrow 6_{6}$ & 90686.38 & 26.1 & $0.133(0.002)$ & $0.079(0.002)$ & \\
\hline & $7_{8} \rightarrow 6_{7}$ & 93870.09 & 19.9 & $0.420(0.002)$ & $0.238(0.002)$ & \\
\hline & $8_{7} \rightarrow 7_{6}$ & 99866.50 & 28.1 & $0.119(0.002)$ & $0.070(0.002)$ & \\
\hline & $8_{8} \rightarrow 7_{7}$ & 103640.75 & 31.1 & $0.119(0.002)$ & $0.059(0.003)$ & \\
\hline & $8_{9} \rightarrow 7_{8}$ & 106347.73 & 25.0 & $0.286(0.002)$ & $0.156(0.002)$ & \\
\hline & $9_{8} \rightarrow 8_{7}$ & 113410.20 & 33.6 & $0.077(0.002)$ & $0.047(0.002)$ & \\
\hline & $9_{9} \rightarrow 8_{8}$ & 116594.78 & 36.7 & $0.090(0.008)$ & $0.056(0.010)$ & \\
\hline & $10_{11} \rightarrow 9_{10}$ & 131551.96 & 37.0 & $0.084(0.004)$ & $0.066(0.005)$ & \\
\hline & $11_{10} \rightarrow 10_{9}$ & 140180.74 & 46.4 & $0.032(0.005)$ & $0.016(0.005)$ & Red wing \\
\hline & $11_{11} \rightarrow 10_{10}$ & 142501.69 & 49.7 & $0.014(0.004)$ & $0.013(0.005)$ & \\
\hline & $11_{12} \rightarrow 10_{11}$ & 144244.82 & 43.9 & $0.067(0.004)$ & & \\
\hline & $12_{13} \rightarrow 11_{12}$ & 156981.65 & 51.5 & $0.032(0.007)$ & & \\
\hline \multirow[t]{3}{*}{$\mathrm{CC}^{34} \mathrm{~S}$} & $7_{8} \rightarrow 6_{7}$ & 91913.53 & 19.5 & $0.015(0.003)$ & $0.011(0.003)$ & \\
\hline & $8_{8} \rightarrow 7_{7}$ & 101371.04 & 30.6 & $0.005(0.001)$ & & \\
\hline & $8_{9} \rightarrow 7_{8}$ & 104109.33 & 24.5 & $0.006(0.002)$ & $0.006(0.002)$ & \\
\hline \multirow[t]{5}{*}{$\mathrm{CCCS}$} & $15 \rightarrow 14$ & 86708.38 & 33.3 & $0.052(0.003)$ & $0.205(0.003)$ & overlapped with $\mathrm{HCO}$ \\
\hline & $16 \rightarrow 15$ & 92488.49 & 37.7 & $0.034(0.003)$ & $0.022(0.003)$ & \\
\hline & $17 \rightarrow 16$ & 98268.52 & 42.4 & $0.021(0.002)$ & $0.015(0.002)$ & \\
\hline & $18 \rightarrow 17$ & 104048.45 & 47.4 & $0.017(0.002)$ & & \\
\hline & $19 \rightarrow 18$ & 109828.29 & 52.7 & $0.015(0.003)$ & & \\
\hline $\mathrm{C}^{13} \mathrm{CCS}$ & $15 \rightarrow 14$ & 85838.10 & 33.0 & $0.006(0.002)$ & & \\
\hline $\mathrm{o}-\mathrm{H}_{2} \mathrm{~S}$ & $1_{1,0} \rightarrow 1_{0,1}$ & 168762.75 & 8.1 & $0.892(0.016)$ & $0.381(0.019)$ & Red wing \\
\hline \multirow[t]{10}{*}{ NS } & ${ }^{2} \pi_{1 / 2} 5 / 2_{1,7 / 2} \rightarrow 3 / 2_{-1,5 / 2}$ & 115153.93 & 8.8 & $0.236(0.007)$ & $0.393(0.008)$ & \\
\hline & ${ }^{2} \pi_{1 / 2} 5 / 2_{1,5 / 2} \rightarrow 3 / 2_{-1,3 / 2}$ & 115156.81 & 8.8 & $0.141(0.007)$ & $0.273(0.008)$ & \\
\hline & ${ }^{2} \pi_{1 / 2} 5 / 2_{1,3 / 2} \rightarrow 3 / 2_{-1,1 / 2}$ & 115162.98 & 8.8 & $0.049(0.011)$ & $0.166(0.013)$ & Bad baseline \\
\hline & ${ }^{2} \pi_{1 / 2} 5 / 2_{1,3 / 2} \rightarrow 3 / 2_{-1,3 / 2}$ & 115185.34 & 8.8 & $0.040(0.009)$ & & \\
\hline & ${ }^{2} \pi_{1 / 2} 5 / 2_{1,5 / 2} \rightarrow 3 / 2_{-1,5 / 2}$ & 115191.46 & 8.8 & & $0.068(0.012)$ & \\
\hline & ${ }^{2} \pi_{1 / 2} 5 / 2_{-1,5 / 2} \rightarrow 3 / 2_{1,5 / 2}$ & 115489.41 & 8.9 & $0.067(0.009)$ & & \\
\hline & ${ }^{2} \pi_{1 / 2} 5 / 2_{-1,3 / 2} \rightarrow 3 / 2_{1,3 / 2}$ & 115524.60 & 8.9 & $0.036(0.009)$ & & \\
\hline & ${ }^{2} \pi_{1 / 2} 5 / 2_{-1,7 / 2} \rightarrow 3 / 2_{1,5 / 2}$ & 115556.25 & 8.9 & $0.471(0.010)$ & & \\
\hline & ${ }^{2} \pi_{1 / 2} 5 / 2_{-1,5 / 2} \rightarrow 3 / 2_{1,3 / 2}$ & 115570.76 & 8.9 & $0.318(0.009)$ & & \\
\hline & ${ }^{2} \pi_{1 / 2} 5 / 2_{-1,3 / 2} \rightarrow 3 / 2_{1,1 / 2}$ & 115571.95 & 8.9 & $0.194(0.008)$ & & \\
\hline
\end{tabular}



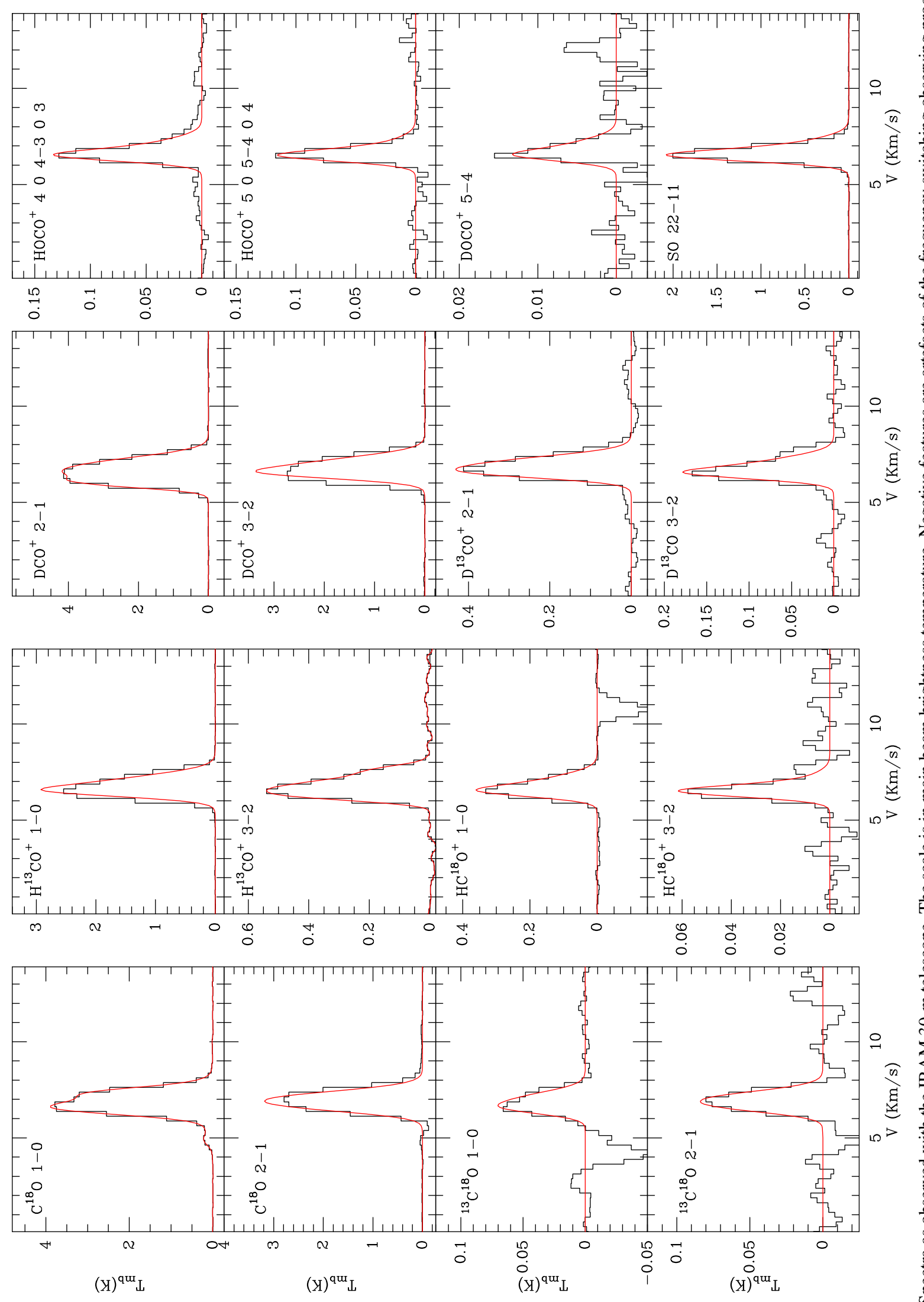

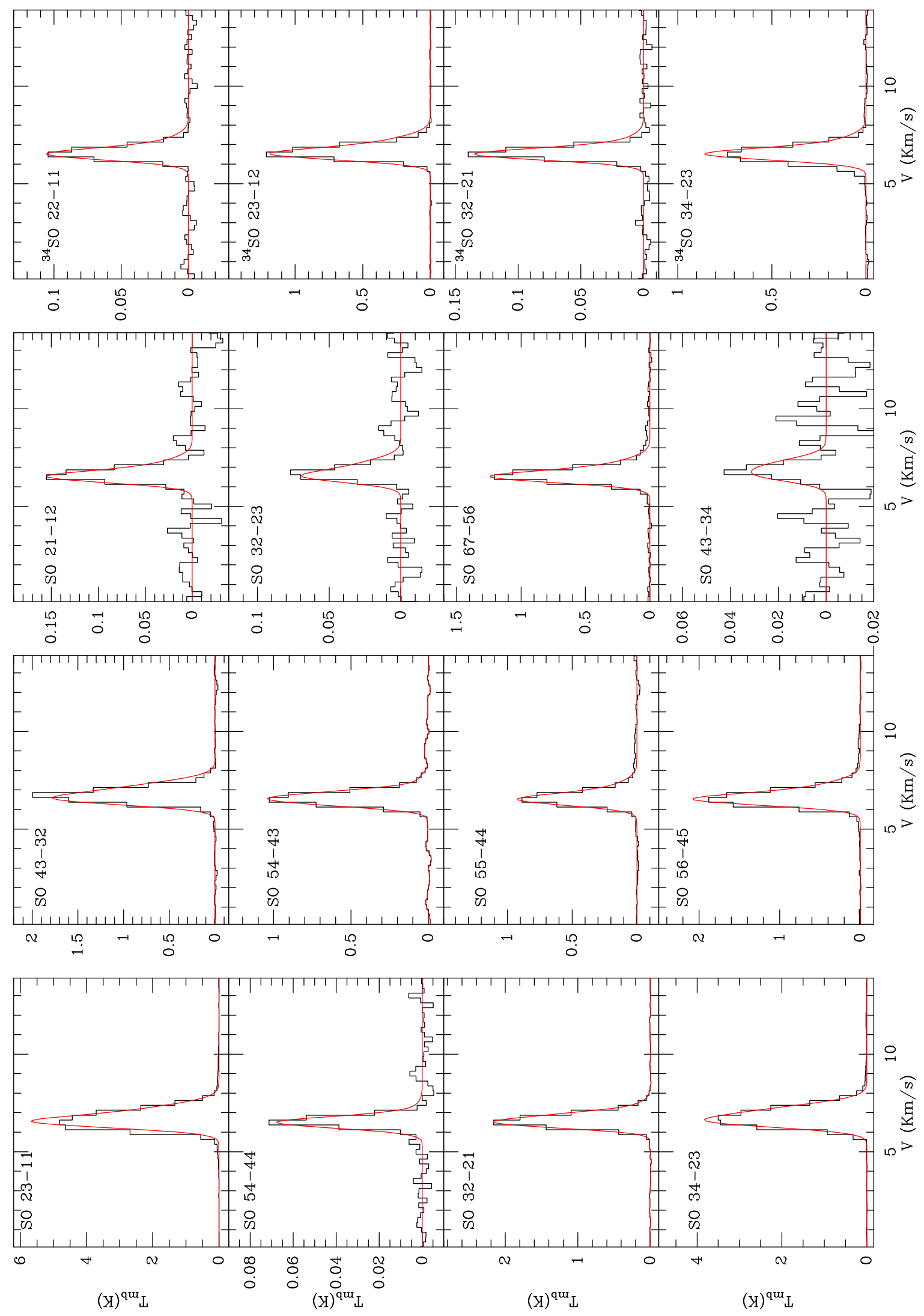

ف묘. 

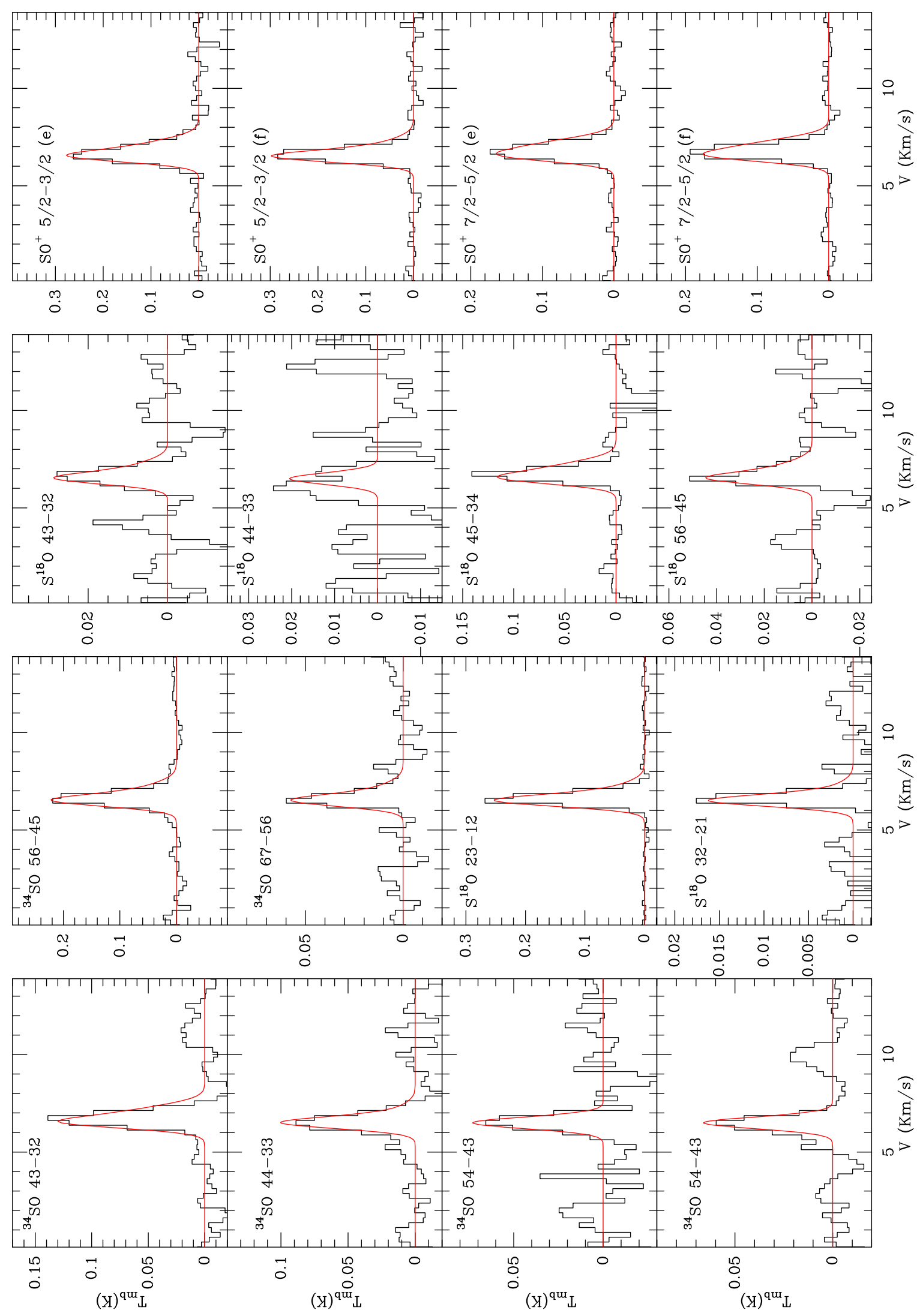

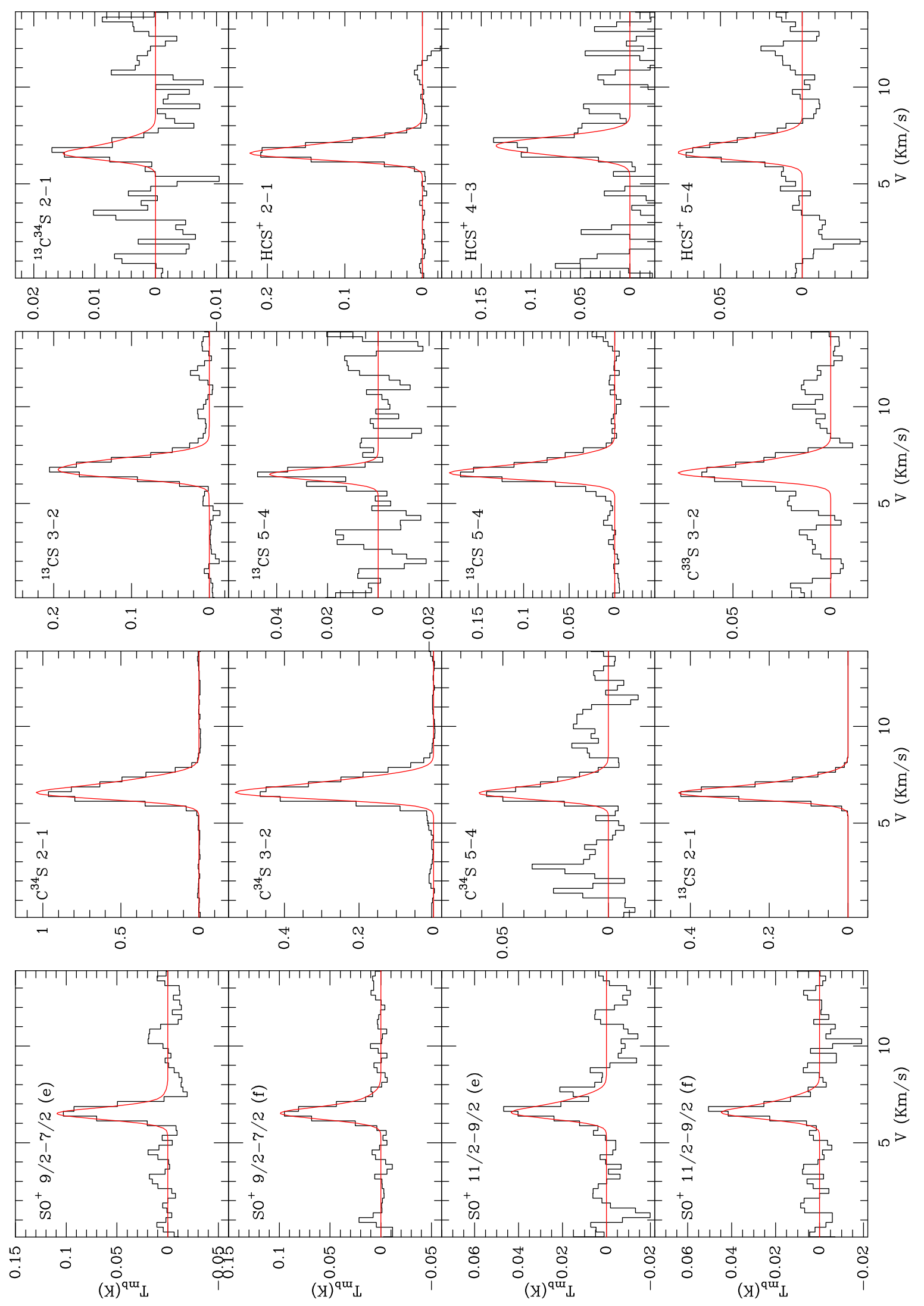

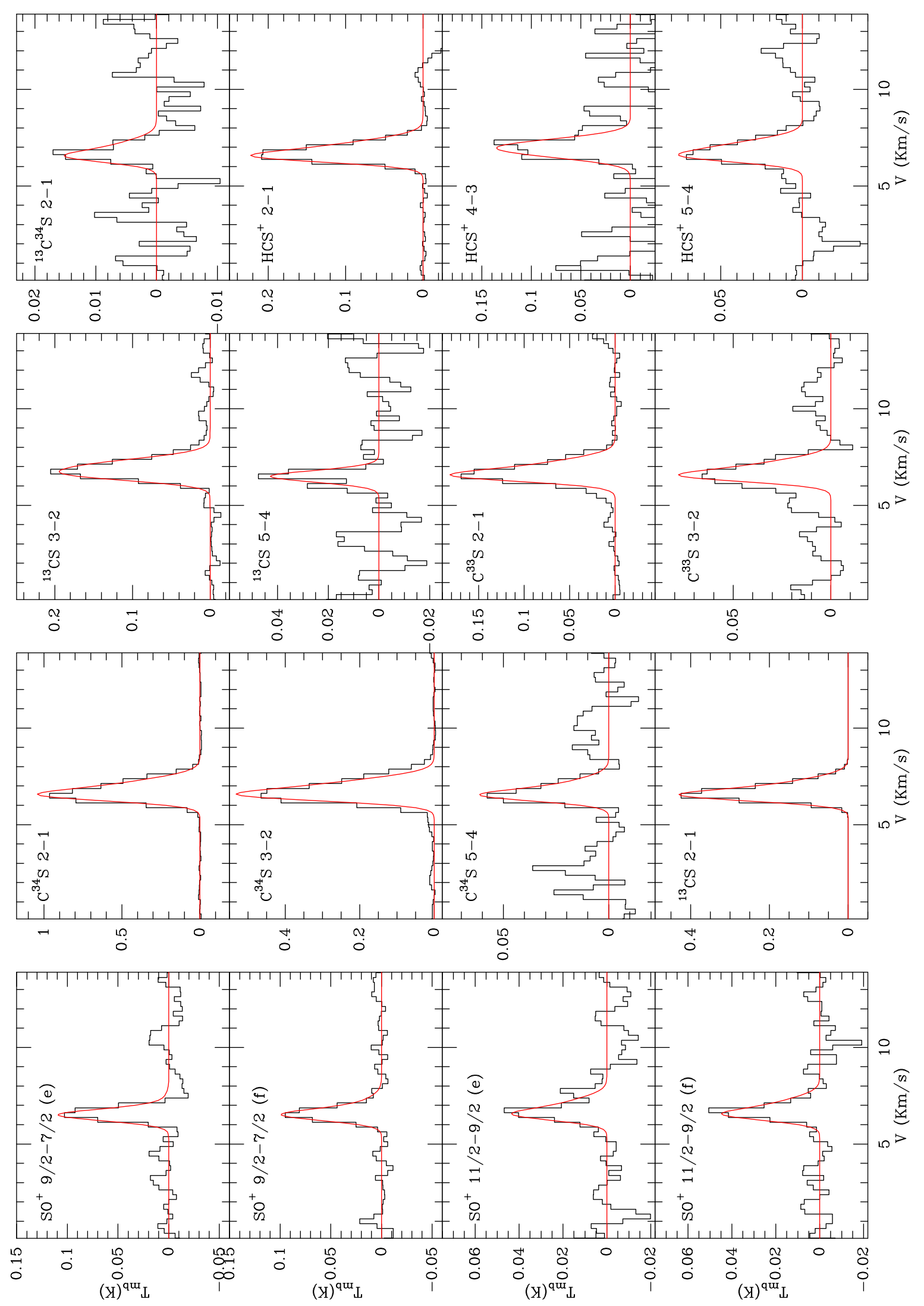
A. Fuente et al.: Ionization fraction and the enhanced sulfur chemistry in Barnard 1
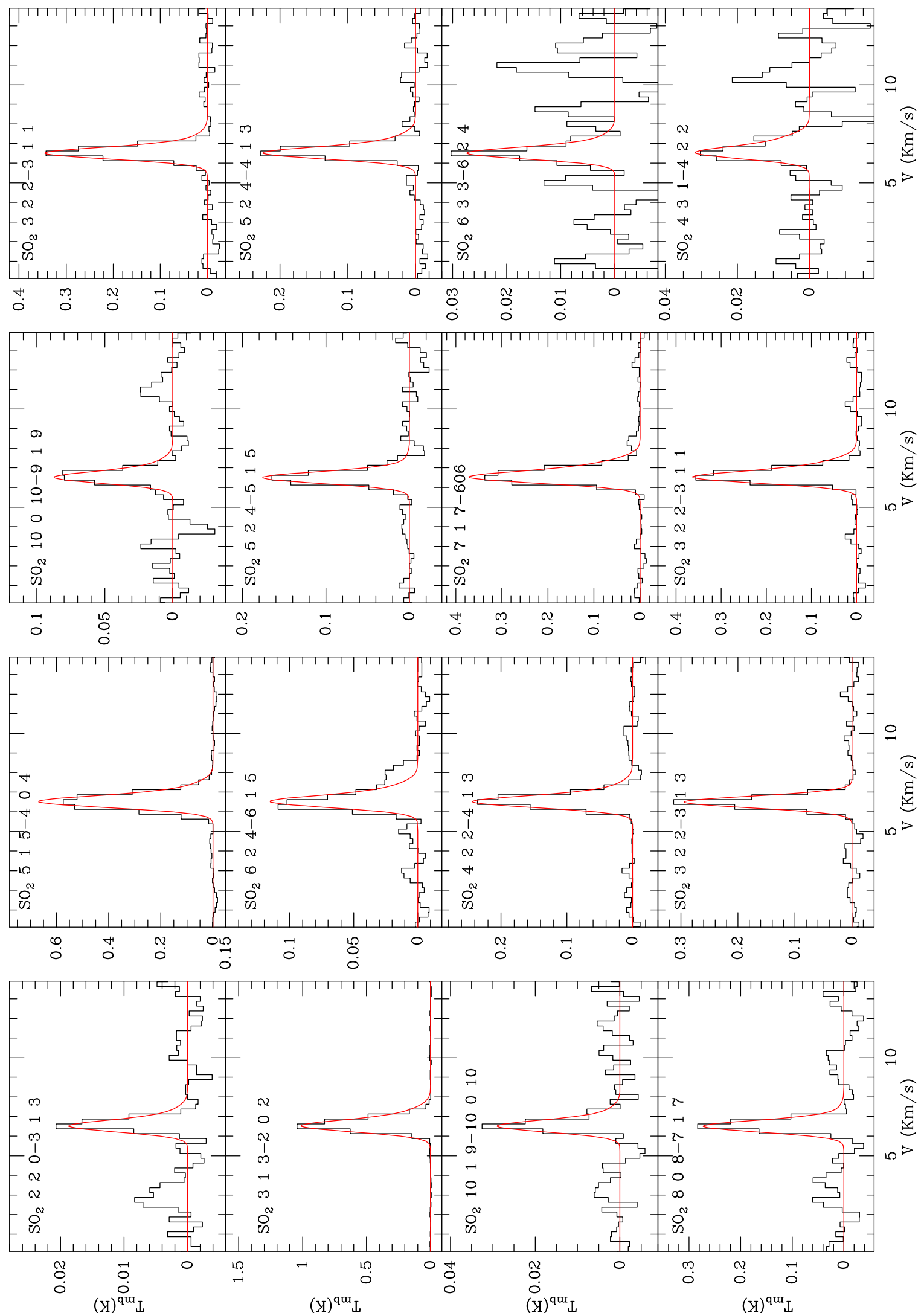

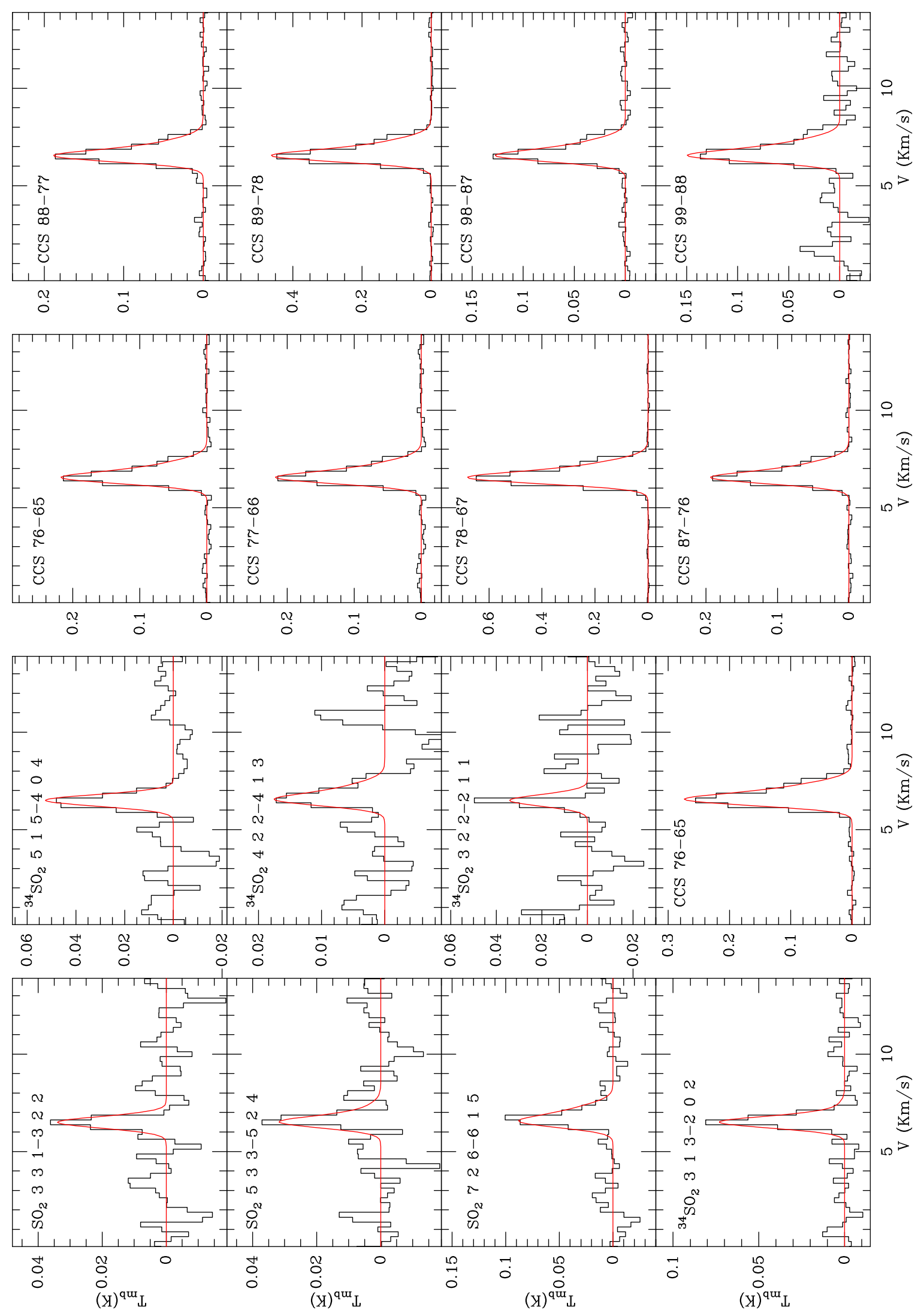
A. Fuente et al.: Ionization fraction and the enhanced sulfur chemistry in Barnard 1
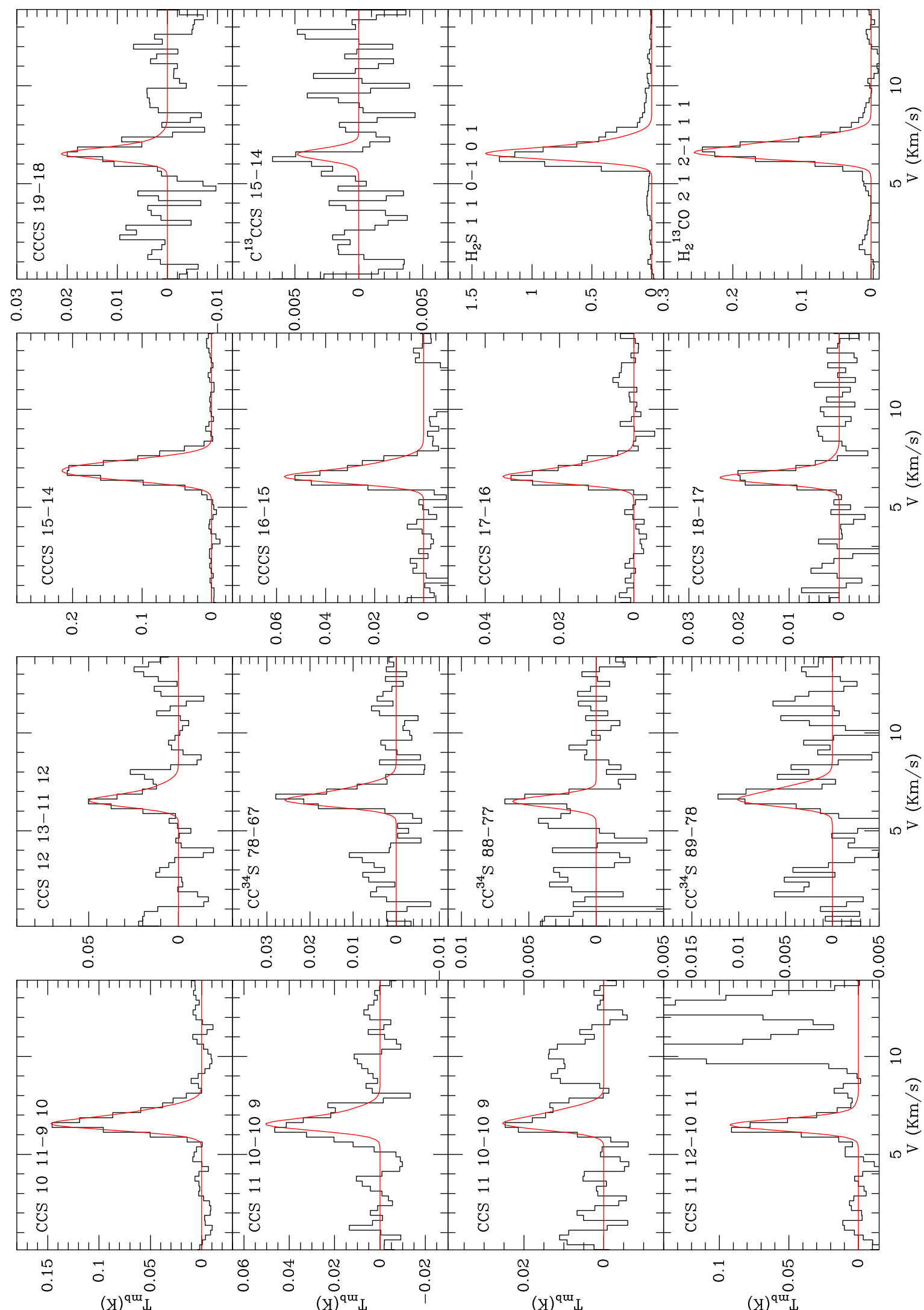
A\&A 593, A94 (2016)
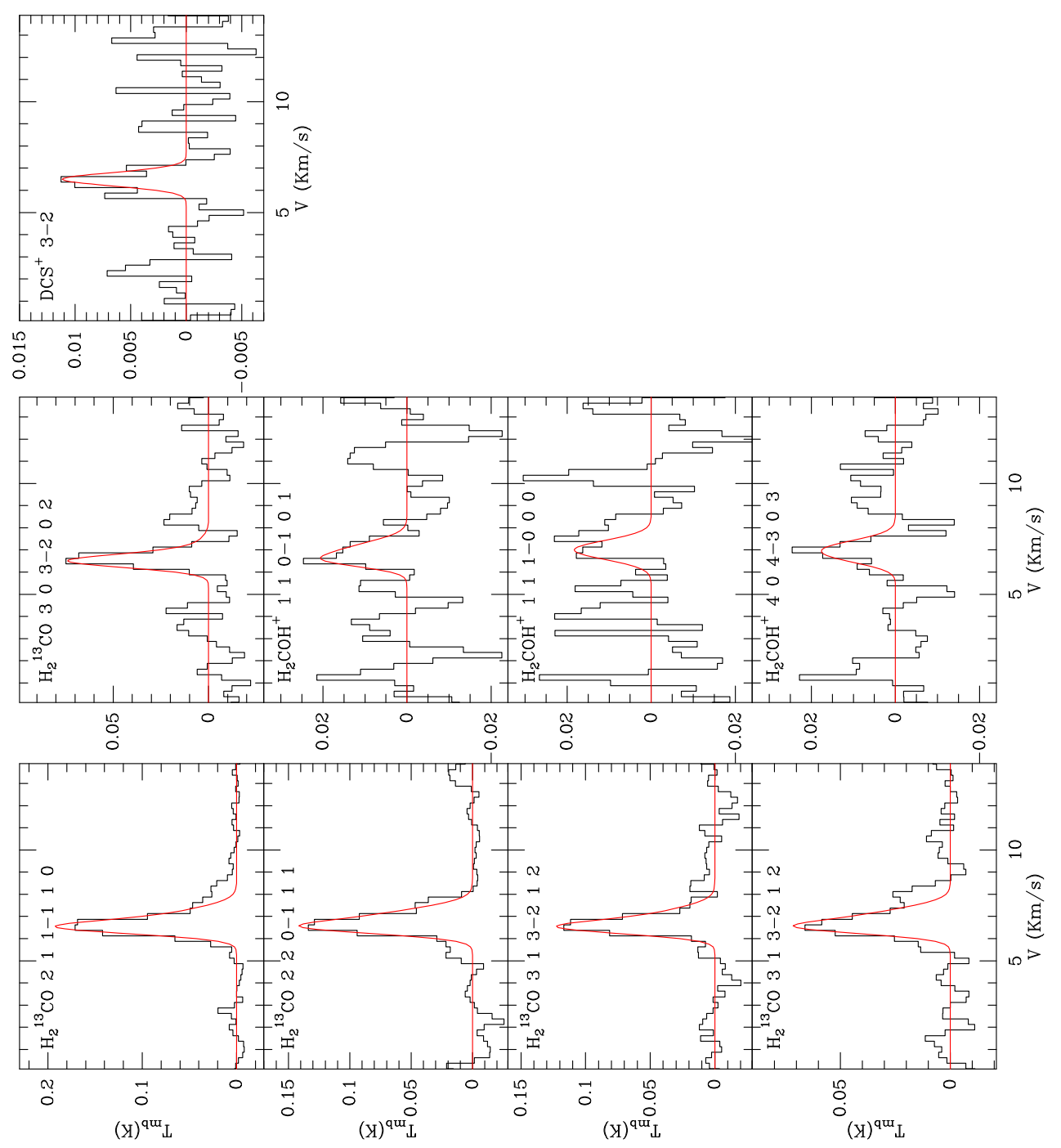

离

A94, page 26 of 30 
A. Fuente et al.: Ionization fraction and the enhanced sulfur chemistry in Barnard 1
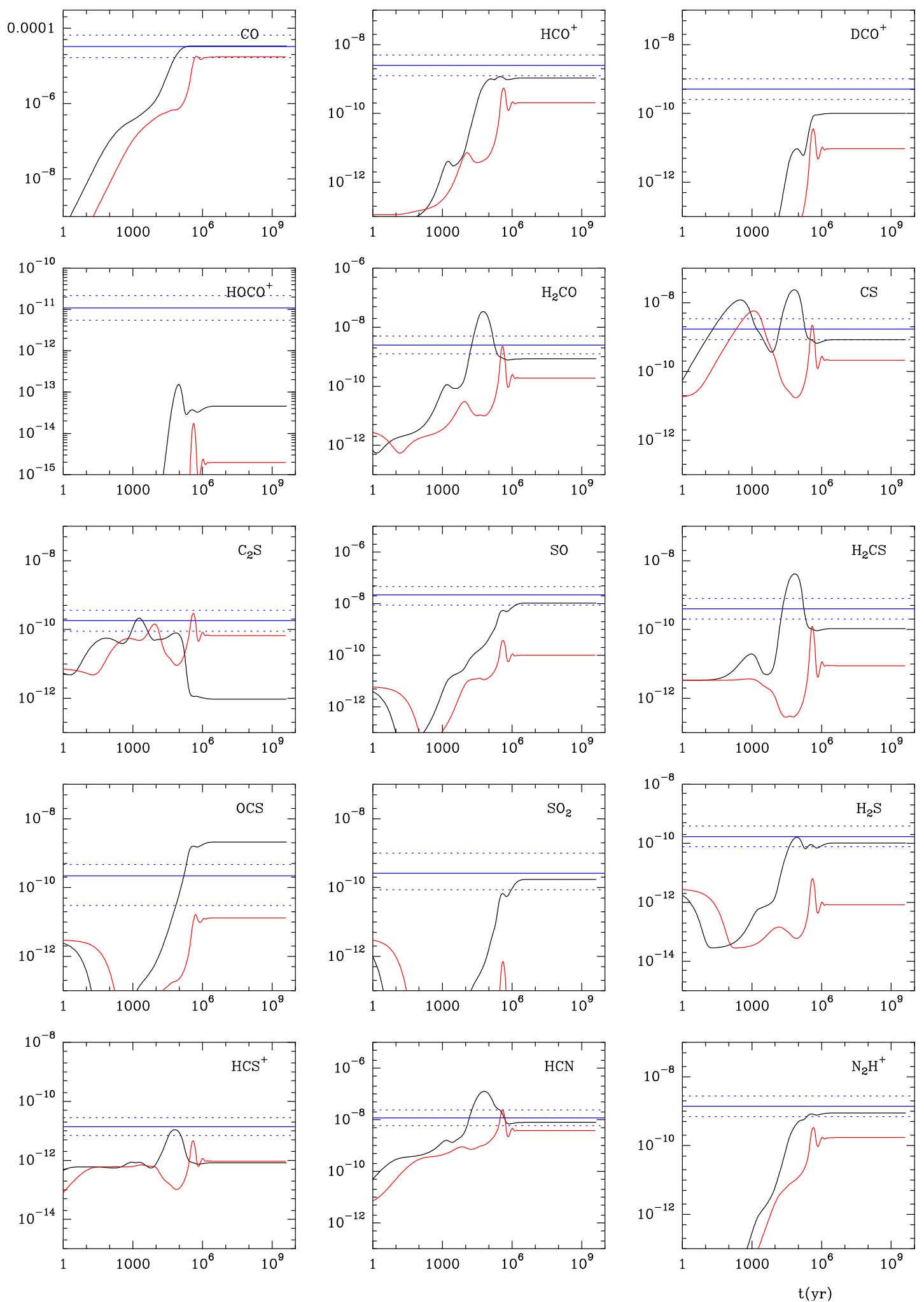

Fig. B.2. Results of our best-fit model $\left(\zeta_{\mathrm{H}_{2}}=4.0 \times 10^{-17} \mathrm{~s}^{-1}, \mathrm{O} / \mathrm{H}=3.0 \times 10^{-5}, \mathrm{~N} / \mathrm{H}=6.4 \times 10^{-5}, \mathrm{C} / \mathrm{H}=1.7 \times 10^{-5}\right.$, and $\left.\mathrm{S} / \mathrm{H}=6.0 \times 10^{-7}\right)$. We assume a density of $n\left(\mathrm{H}_{2}\right)=10^{5} \mathrm{~cm}^{-3}$ and $T_{k}=12 \mathrm{~K}$. The thick and dashed blue lines indicate the observed values and their uncertainty, which are considered to be a factor of 2 for most molecules (see text), respectively. In the case of $\mathrm{SO}, \mathrm{SO}_{2}$, and OCS, their abundances change by more than a factor of 2 between the $6.5 \mathrm{~km} \mathrm{~s}^{-1}$ and $7.0 \mathrm{~km} \mathrm{~s}^{-1}$ velocity components. In the panels corresponding to these species, the dashed lines indicate the abundance for each component separately. In red we show model calculations for the same conditions and $n\left(\mathrm{H}_{2}\right)=10^{4} \mathrm{~cm}^{-3}$. 
A\&A 593, A94 (2016)
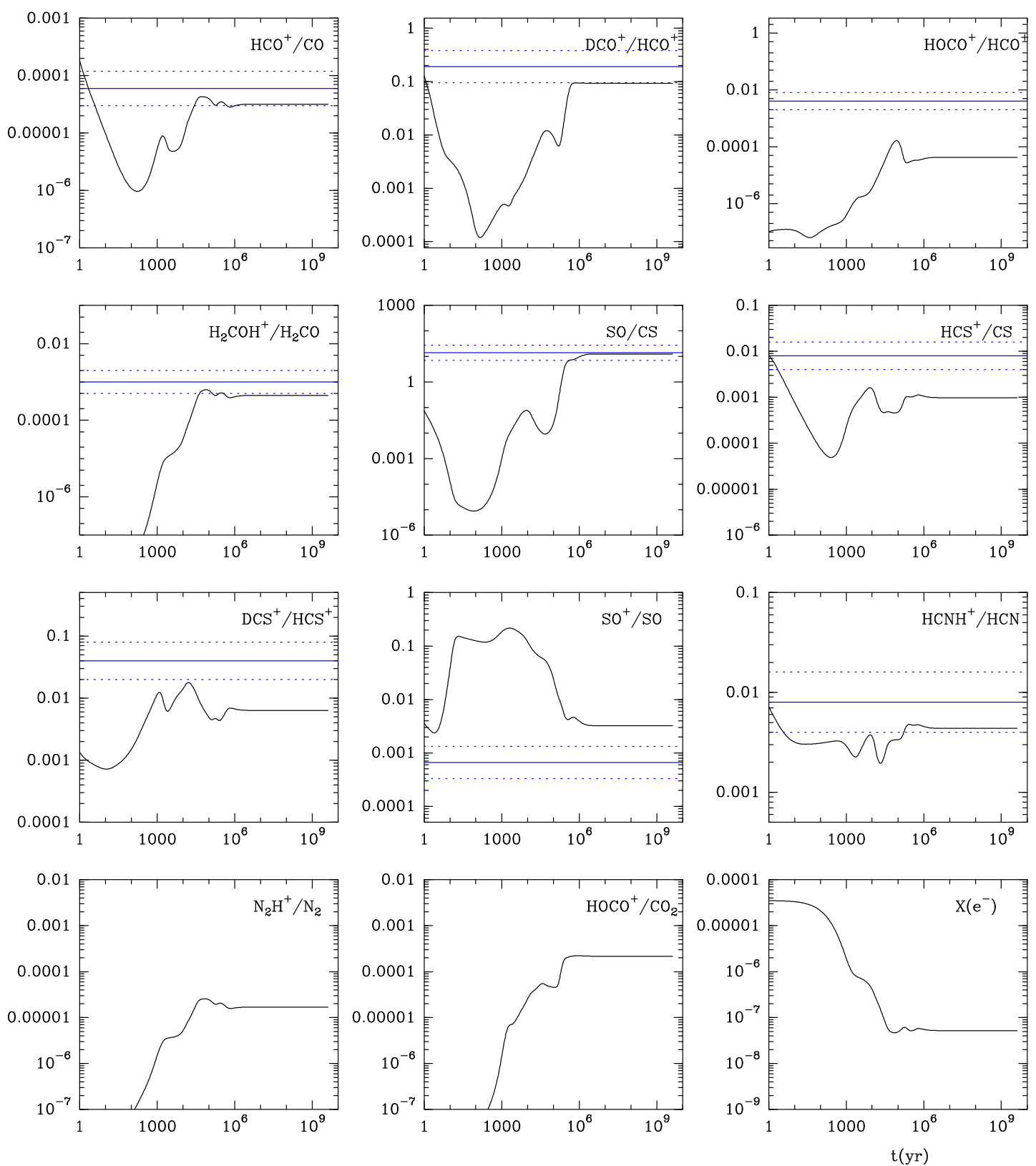

Fig. B.3. Results of our best-fit model $\left(\zeta_{\mathrm{H}_{2}}=4.0 \times 10^{-17} \mathrm{~s}^{-1}, \mathrm{O} / \mathrm{H}=3.0 \times 10^{-5}, \mathrm{~N} / \mathrm{H}=6.4 \times 10^{-5}, \mathrm{C} / \mathrm{H}=1.7 \times 10^{-5}\right.$, and $\left.\mathrm{S} / \mathrm{H}=6.0 \times 10^{-7}\right)$. We assume a density of $n\left(\mathrm{H}_{2}\right)=10^{5} \mathrm{~cm}^{-3}$ and $T_{k}=12 \mathrm{~K}$. The thick and dashed blue lines indicate the observed values and their uncertainties. 
A. Fuente et al.: Ionization fraction and the enhanced sulfur chemistry in Barnard 1
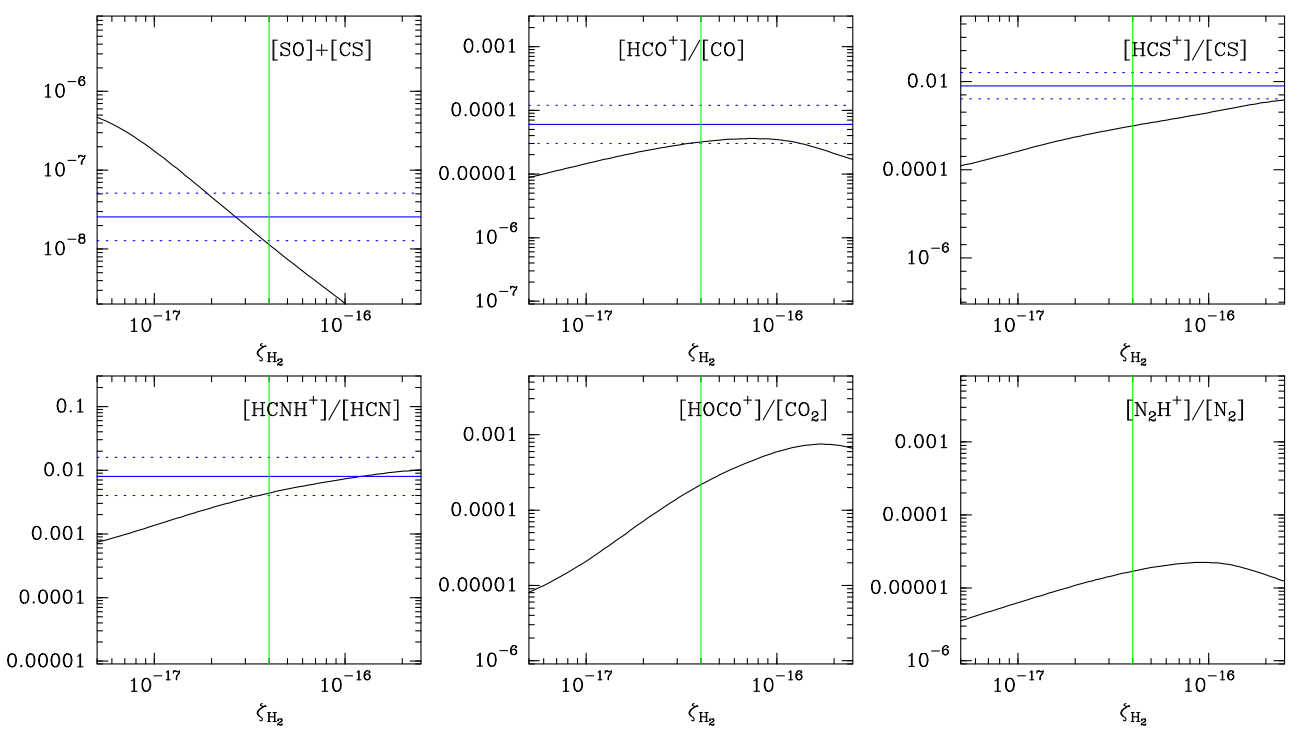

Fig. B.4. Steady-state calculations of fractional abundances and abundance ratios. All the input parameters except $\zeta_{\mathrm{H}_{2}}$ are the same as in Fig. B.2. Horizontal blue lines correspond to the observed values and their uncertainty that is considered a factor of 2 . Vertical green lines indicate the estimated value of the cosmic ray ionization rate, $\zeta_{\mathrm{H}_{2}}=4 \times 10^{-17} \mathrm{~s}^{-1}$.
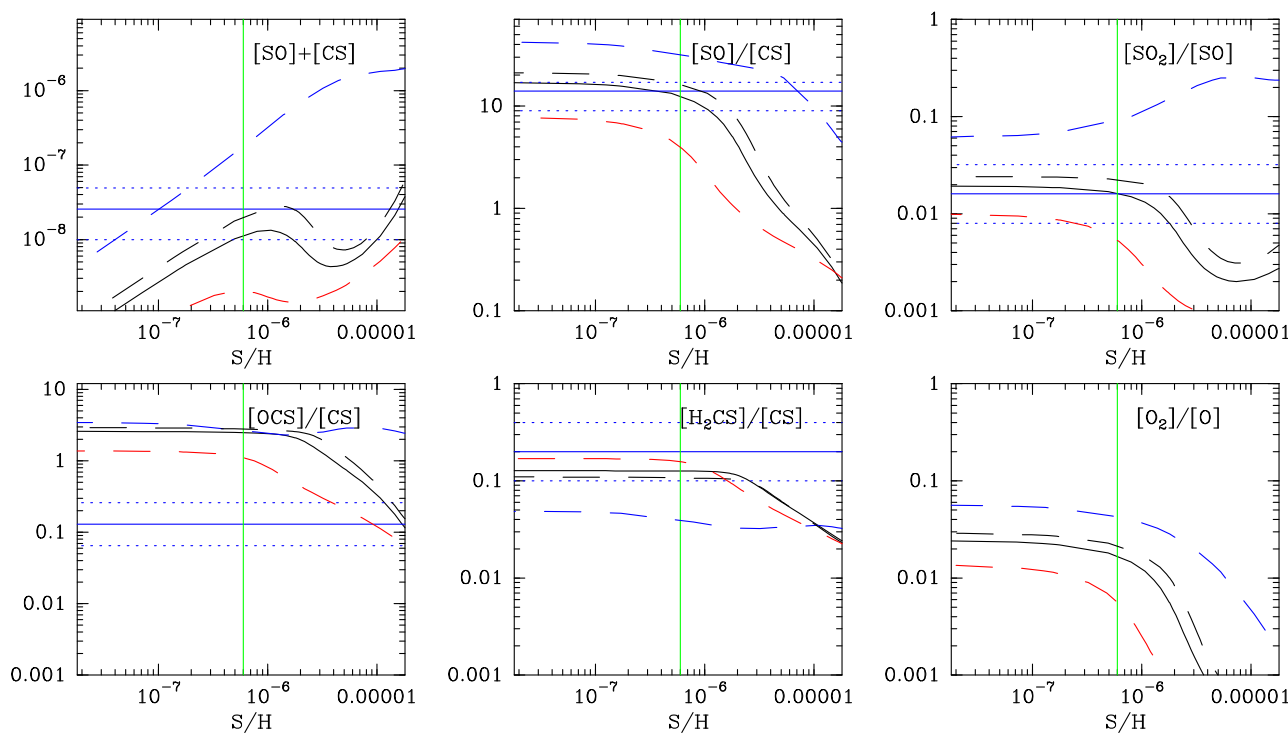

Fig. B.5. Steady-state calculations of fractional abundances and abundance ratios. All the input parameters except S/H are the same as in Fig. B.2. Horizontal blue lines correspond to the observed values and their uncertainty. Vertical green lines indicate the estimated value of the sulfur elemental abundance, $\mathrm{S} / \mathrm{H}=6 \times 10^{-7}$. The solid black line represents our best model fit, $\zeta_{\mathrm{H}_{2}}=4 \times 10^{-17} \mathrm{~s}^{-1}$. The dashed blue, black, and red lines correspond to $\zeta_{\mathrm{H}_{2}}=1,4$ and $10 \times 10^{-17} \mathrm{~s}^{-1}$, respectively. 
A\&A 593, A94 (2016)
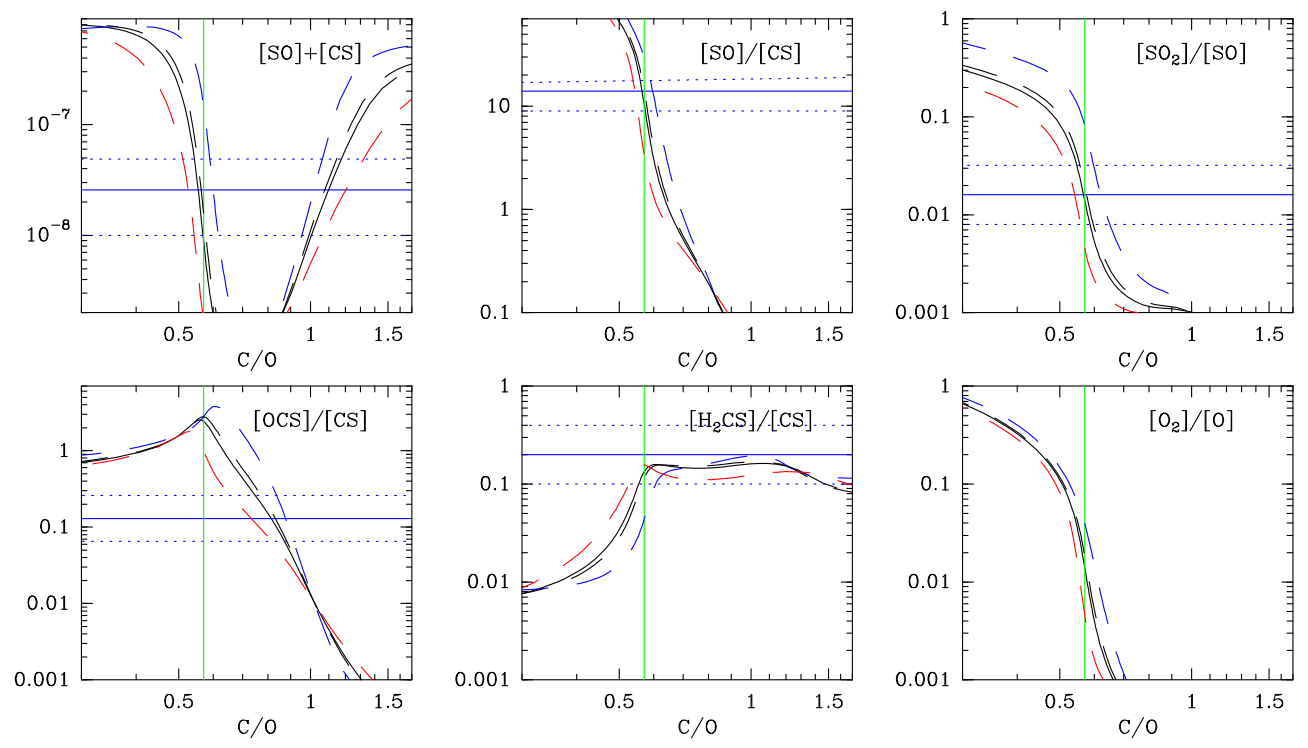

Fig. B.6. Steady-state calculations of fractional abundances and abundance ratios. All the input parameters except O/H are the same as in Fig. B.2. Horizontal blue lines correspond to the observed values and their uncertainty. Vertical green lines indicate the estimated value of $\mathrm{C} / \mathrm{O}$ elemental ratio, $\mathrm{C} / \mathrm{O}=0.6$. The dashed blue, black, and red lines correspond to $\zeta_{\mathrm{H}_{2}}=1,4$ and $10 \times 10^{-17} \mathrm{~s}^{-1}$, respectively. 